\title{
Live Cell STED Microscopy Using Genetically Encoded Markers
}

\author{
Dissertation \\ zur Erlangung des Doktorgrades \\ der Mathematisch-Naturwissenschaftlichen Fakultäten \\ der Georg-August-Universität zu Göttingen
}

vorgelegt von

Birka Hein

aus Celle

Göttingen 2009 
D7

Referent: $\quad$ Prof. Dr. Jürgen Troe

Korreferent: Prof. Dr. Stefan W. Hell

Tag der mündlichen Prüfung: 


\section{Abstract}

Far-field fluorescence microscopy is a tool of outstanding importance in the biological sciences, due to its non-invasiveness especially for the investigation of living cells, tissue and animals. Today also the major drawback in light microscopy, namely its limited resolution, has been overcome by utilizing photoswitching between different states of the fluorophore. STimulated Emission Depletion (STED) microscopy was the first farfield fluoresence nanoscopy approach to evolve, offering images with a resolution far beyond the diffraction barrier. However, until now STED microscopy was mostly confined to imaging fixed and therefore dead samples, because immunocytochemistry, the method of choice for fluorescence labeling for STED microscopy, requires in most cases the permeabilization and therefore fixation of the specimen.

In this work, STED microscopy in living cells has been established. Two different labeling approaches have been applied: By using derivatives of green fluorescent protein (GFP), which is widely used in conventional live cell imaging, a resolution of $50 \mathrm{~nm}$ in the lateral and $150 \mathrm{~nm}$ in the axial direction within living cells is achieved. Alternatively, genetically encoded tagging proteins capable of binding modified organic dyes are employed. Structures as small as $40 \mathrm{~nm}$ can be discerned in living cells by this method. Both approaches also allow for watching structural changes on the nanoscale within the cell by taking time-lapse image series, as shown in various examples in this work. To monitor even faster processes on the nanoscale, STED fluorescence correlation spectroscopy (FCS) is applied in conjunction with these labeling approaches, elucidating nanoscopic details of the dynamics of membraneanchored proteins not observable with conventional confocal FCS.

By utilizing these results, many ongoing fluorescence microscopy studies in living cells, which are currently performed with a diffraction-limited confocal microscope, can be carried out with a substantially improved spatial resolution in all directions by means of STED microscopy. Altogether, this paves the way for new insights into a bustling nanocosmos. 


\section{Contents}

1 Introduction 1

$1.1 \quad$ History of Light Microscopy $\ldots \ldots \ldots \ldots$. . . . . . . . . . . . 1

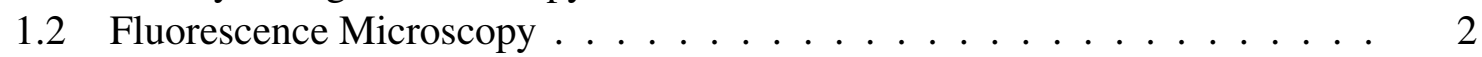

1.3 Fluorescence Nanoscopy $\ldots \ldots \ldots \ldots$. . . . . . . . . . . . 4

1.4 Labeling Methods for Fluorescence Imaging . . . . . . . . . . . . . . . 7

$1.4 .1 \quad$ Immunocytochemistry $\ldots \ldots \ldots 7 \ldots$

1.4 .2 Autofluorescent Proteins . . . . . . . . . . . . . . . . . . . 8

1.4 .3 Selflabeling Protein Tags . . . . . . . . . . . . . . . . . . . 9

1.5 Objective of This Work . . . . . . . . . . . . . . . 12

$\begin{array}{lll}2 & \text { Techniques } & 13\end{array}$

2.1 STED Microscopy $\ldots \ldots \ldots \ldots$. . . . . . . . . . . . . . . . . . . . . . . . . . . . . .

2.1 .1 The Microscope . . . . . . . . . . . . . . . . . . . 13

2.1 .2 Shaping the Effective Spot of Excitation $\ldots \ldots$. . . . . . . . 15

2.1 .3 Performance of the STED Microscope . . . . . . . . . . . . . . 16

2.2 Sample Preparation . . . . . . . . . . . . . . . . . . 18

2.2 .1 Plasmid Construction . . . . . . . . . . . . . . . . . . 18

2.2 .2 Biological Systems used in this Work . . . . . . . . . . . . . 19

$2.2 .3 \quad$ Organic Dyes as a Live-Cell Compatible Fluorescent Label . . . . . 20

2.3 Screening of Fluorescent Proteins f . . . . . . . . . . . . 22

3 Live Cell STED Microscopy 24

$3.1 \quad$ Live Cell STED Imaging using Fluorescent Proteins . . . . . . . . . . . . . 25

3.1 .1 Live Cell Imaging in Mammalian Cells . . . . . . . . . . . . . 26

3.1 .2 Axial Resolution Enhancement. . . . . . . . . . . . . . . . . . . . . . . . . . . . . 34

3.1 .3 Imaging Living Yeast . . . . . . . . . . . . . . . . . . . . . 35

$3.2 \quad$ Live Cell STED Imaging using Organic Dyes . . . . . . . . . . . . . . 37

3.2.1 Enzyme-Mediated Labeling using Acyl Carrier Protein . . . . . . . 38

3.2.2 Selflabeling Tags for Intracellular Applications . . . . . . . . . . . . . 40

3.3 A Simplified Setup for Live Cell Imaging using CW-Lasers . . . . . . . . . . . . 46

3.4 The Adequate Objective Lens . . . . . . . . . . . . . . . . . . . . 48

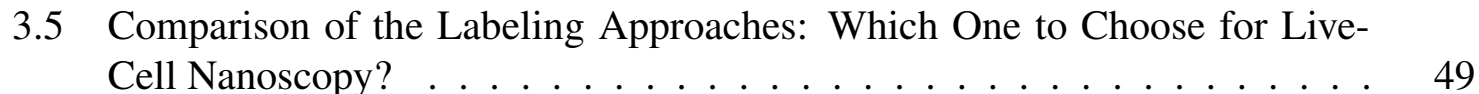


4 Live-Cell STED FCS: Fluorescence Correlation Spectroscopy in Reduced Focal Volumes $\quad 52$

4.1 Theoretical Model of FCS $\ldots \ldots \ldots \ldots \ldots \ldots$

4.2 Combining FCS with STED $\ldots \ldots \ldots \ldots \ldots \ldots$

4.3 STED FCS in Living Cells $\ldots \ldots \ldots \ldots \ldots \ldots$

4.3 .1 Dynamics of GPI-anchored Proteins . . . . . . . . . . . . . 57

4.3 .2 Influence of the Label $\ldots \ldots \ldots \ldots$

5 Conclusion and Outlook 61

\begin{tabular}{ll}
\hline Literature & 64
\end{tabular} 


\section{Introduction}

\subsection{History of Light Microscopy}

The term microscopy is derived from the Greek $\mu \iota \kappa \rho o ́ \varsigma$ and $\sigma \kappa o \pi \epsilon \tilde{\iota} \nu$, meaning small and to view, respectively. As early as the first century A.D., Seneca mentioned the magnification effect of water-filled bowls on the apparent size of objects [1]: literae quamvis minutae et obscurae per vitream pilam aqua plenam maiores clariosque cernuntur. However, he attributed this magnification not to the shape of the bowl but to the water.

The first denoted microscope was built by the two Dutch spectacle makers Zacharias and Hans Janssen around 1590, and consisted of a converging objective and a diverging eye lens. In 1609 Galileo heard of these early experiments, worked out the principles of lenses, and built an enhanced microscope with a focusing device.

Further improvements can be credited to Ernst Abbe, who developed a physical description of the theory of the microscope in 1873 [2]. Most importantly, he realized that the resolution (the minimal distance $d$ at which two objects can be separated from each other) is due to diffraction limited by the wavelength $\lambda$ of the employed light, leading to the wellknown Abbe-formula

$$
d \approx \frac{\lambda}{2 n \sin \alpha}
$$

where $n$ is the refractive index of the embedding medium and $\alpha$ the semiaperture angle of the focused light. With the definition of the numerical aperture NA $=n \sin \alpha$, this means that the minimal size of the focus is governed by the wavelength and the numerical aperture of the optical system. With the help of Carl Zeiss and Otto Schott, in the following years Abbe developed a microscope whose resolution was not limited by the material or the manufacturing process any more, but by this physical barrier.

From these early developments on, light microscopy has become a standard experimental tool for many disciplines, especially in the life sciences.

\section{Light Microscopy in Biology}

Several fundamental principles in biology have been discovered through the use of microscopes. In 1630, details of a bee, concealed to the naked eye, were drawn by Francesco Stelluti [3]. Advancing in parallel with the technical improvements, the imaged details kept getting smaller and smaller. Around 1660, the term cell was coined by Robert Hooke after his microscopic investigations of cork tissue, because the structures he saw - the cell walls - 
reminded him of the cells of a monastery [4]. Later on, organelles and other substructures of the cell were revealed by microscopy as well.

Also today, the microscope is a major tool in biology. A search in Web of Science for the term "microscopy" leads to more than 87000 results just in the year 2008, which emphasizes the importance of the continuing developments in this field.

\subsection{Fluorescence Microscopy}

Since a single cell consists of thousands of proteins, lipids and nucleic acids, a method is needed which selectively highlights the features of interest. A well suited method is the specific staining with a fluorescent dye and the subsequent visualization using a fluorescence microscope.

To achieve this, the specimen is illuminated with light of an appropriate wavelength. The fluorescent dye absorbs the light, going from the electronic ground state $S_{0}$ to an electronically excited state $S_{1}$. After radiationless decay into the vibrational groundstate, eventually redshifted (lower-energy) fluorescence is emitted, which can be separated from the excitation light using dichroic mirrors.

\section{Confocal Microscopy}

One elaborate mode of operation of a fluorescence microscope is a confocal microscope. Originally patented by Marvin Minsky in 1957, it took another thirty years and the development of lasers as a strong lightsource for confocal microscopy to become a standard technique toward the end of the 1980s [5] [6]. The principle is illustrated in figure 1.1 .

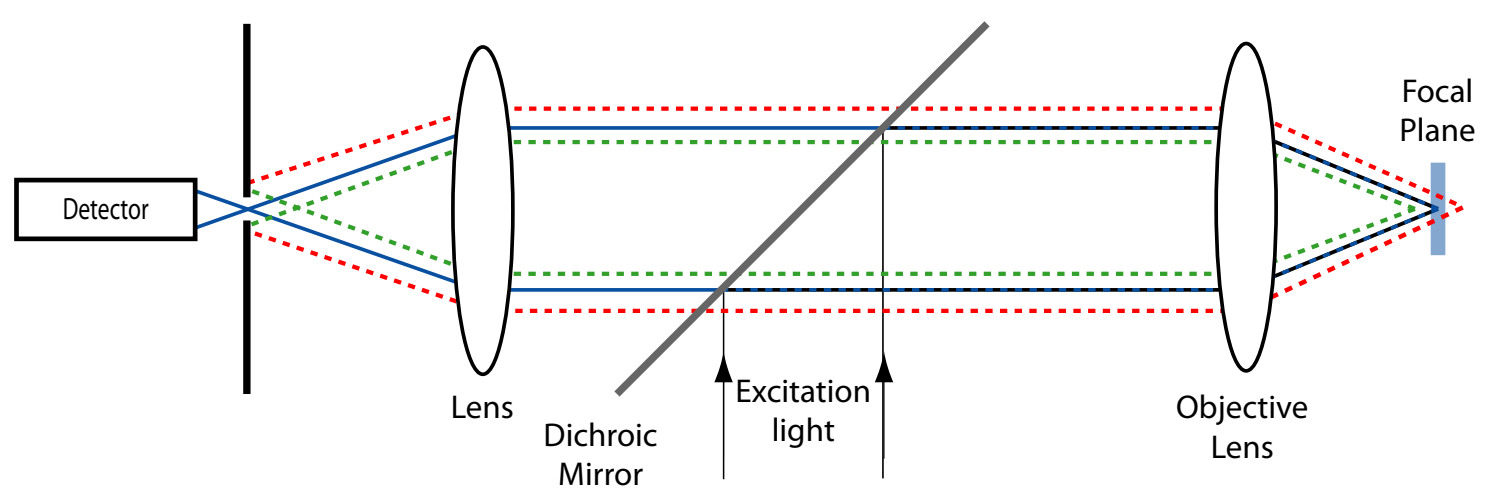

Figure 1.1: Principle of a confocal microscope. The pinhole in front of the detector blocks all emitted light not originating from the focal plane, which leads to background suppression and the ability for optical sectioning. 
Light (nowadays mostly from a laser source) is focused into the specimen via an objective lens. Fluorescence is collected with the same lens, separated from the excitation light by a dichroic mirror and focused onto a pointlike detector, which is often realized by a pinhole in front of a photomultiplier tube (PMT) or an avalanche photodiode (APD). The pinhole suppresses background signal from areas not in the focal plane, as can be seen by the dotted lines in figure 1.1. Only light originating from the focus is properly focused onto the pinhole and therefore sensed by the detector, whereas fluorescence emanating from a different plane is efficiently blocked by the pinhole. This background suppression leads to the optical sectioning capability of confocal microscopy, which is especially important when imaging thick samples. Images are acquired by translating the sample through the excitation spot or by scanning the spot through the sample (Confocal Laser Scanning Microscopy, CLSM), which allows for higher acquisition speeds [7] [8].

Since confocal microscopy has become the gold standard in conventional high resolution microscopy, it is used as a reference throughout this work. 


\subsection{Fluorescence Nanoscopy}

Although fluorescence microscopy is a very versatile tool especially for studying biological systems, it has a major drawback, as Abbe pointed out already more than 100 years ago: The resolution is limited.

Even though other techniques like electron microscopy (EM) [9] [10] or nearfield scanning optical microscopy (NSOM) [11] [12] offer a better, up to atomic resolution, most experiments are still performed using far-field light microscopy, since only there can also the interior of living samples be examined.

In the last 15 years, several techniques have been developed by which the diffraction limit can be broken in far-field optical microscopy [13]. They all rely on a (reversible) switching of fluorescent molecules between a bright (on) and a dark (off) state, using a variety of transitions and different readout-methods [14]. These techniques can be categorized into two groups: the targeted readout methods, which include STED (STimulated Emission Depletion) microscopy as the most prominent example of the RESOLFT (REversible Saturated OpticaL Fluorescence Transition) concept, and the stochastic methods, including PALM (PhotoActivation Localization Microscopy) and STORM (STochastic Optical Reconstruction Microscopy). Their principles will be explained in the following paragraphs.

\section{Breaking the Diffraction Barrier using Stimulated Emission Depletion}

As theoretically proposed in 1994 [15] and soon after also experimentally demonstrated [16], stimulated emission depletion (STED) is a suitable tool for breaking the diffraction barrier. Here, the size of the effectively exciting focus is reduced by switching off the fluorescence ability of the fluorophores in the outer parts of the excitation focus. In STED, switching is implemented by the forced transition between the electronically excited state and the electronic ground state of the fluorophore, being on- and off-state, respectively.

Figure 1.2 describes this process in detail: A fluorophore is optically excited to the first electronically excited state $S_{1}^{v i b}$. From there, fast internal relaxation to the vibrational groundstate $S_{1}$ takes place within a few picoseconds. Spontaneous transition to the electronic groundstate $S_{0}^{v i b}$ under the emission of fluorescence can now occur. Depending on the lifetime of the fluorophore, this process takes place in the nanosecond time regime. However, the fluorophore can also be deexcited by stimulated emission: upon incidence of a photon which matches the energy gap between $S_{1}$ and $S_{0}^{v i b}$, emission of a second photon of the same wavelength can be stimulated, thereby deexciting the fluorophore. This process is also the key element in laser action. The spectral range of the detector is chosen in a way that these photons are not detected; therefore, this process basically switches off the fluorophore. Since fast spontaneous relaxation from $S_{0}^{v i b}$ to $S_{0}$ occurs, a complete depopulation of the electronically excited (on) state can be achieved.

To use this effect for resolution enhancement, the deexcitation of the fluorophores has to be spatially restricted to the outer part of the excitation focus. Therefore, the excitation and STED beams are spatially overlaid. The shape of the STED focus has to be altered in a way that it features a center with zero intensity, and high intensities at the periphery. At the 


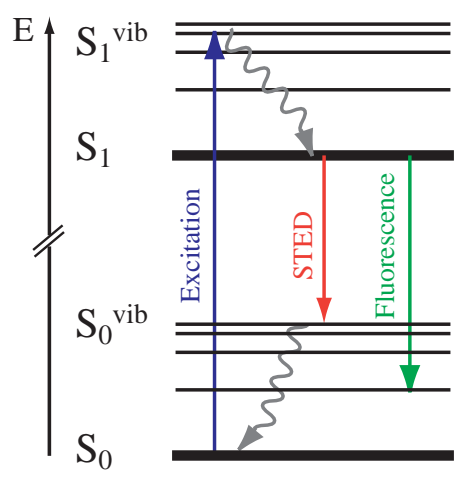

Figure 1.2: Jablonski-diagram showing a fluorophore's energy states involved in STED.

center, where no deexcitation takes place, fluorescence can originate upon excitation. The high STED intensities at the periphery lead to the off-switching of the fluorophores, thereby restricting the effective fluorescence generation to a subdiffraction-sized volume at the very center. Focus-engineering can be achieved by introducing a phaseshift into the STED beam. A suitable pattern will lead to destructive interference at the center upon focusing, therefore resulting in zero intensity, and constructive interference at the periphery. Different phase patterns can be used; for resolution enhancement in the lateral plane, now mostly a helical phaseramp is employed, which leads to a doughnut-shaped focus. Other patterns can also be used, leading for example to resolution enhancement along the optical axis as well.

This technique can also be combined with other advanced microscopy techniques. For example, by implementing STED in a 4Pi-microscope, which improves the resolution along the optical axis by coherently superposing the wavefronts of two opposing objective lenses, recently a uniformly sized focus with a diameter of less than $45 \mathrm{~nm}$ was achieved [17].

The higher the applied STED intensity, the more efficient the deexcitation will also be close to the center. The resolution obtainable by this means can be calculated as

$$
d \approx \frac{\lambda}{2 n \sin \alpha \cdot \sqrt{1+I / I_{s a t}}}
$$

which can be seen as an extension of Abbe's equation 1.1, considering the applied STED intensity $I$. The saturation intensity $I_{\text {sat }}$ is a wavelength- and dye-specific parameter, corresponding to the STED intensity needed to suppress the fluorescence signal to half the original value [18].

As can be derived from equation 1.2 , by increasing the STED intensity, the resolution can in principle be enhanced infinitely, down to molecular sizes or even below. In practice, so far mainly the limited photostability of the fluorophore was the restricting factor. However, by using non-bleaching nitrogen-vacancies in diamond as the fluorophore, recently a resolution below $6 \mathrm{~nm}$ was achieved [19]. 
Technically, the STED microscope can be realized as a two-color confocal microscope with the STED beam being the second color. Confocalization is not required, but conveniently provides background suppression. Also other advantages of confocal microscopy are provided, including the high imaging speed [20], the possibility of arbitrarily choosing the focal plane within the sample [21], the capability of multicolor experiments [22] and also live cell imaging [23].

\section{Other targeted readout methods}

In addition to $S_{0}$ and $S_{1}$, other possibilities exist for the nature of the on- and off-states, whose usage gives rise to different realizations of the RESOLFT concept.

In GSD (Ground State Depletion), a metastable dark state, most commonly the tripletstate $T_{1}$ is used as the off-state [24] [25]. Saturated transition from the fluorescent state $S_{1}$ to the dark state leads to superresolution images. Another possibility is to use two molecular states like cis- and trans or open- and closed rings, where one of the states is fluorescent (on) and the other one is dark (off). The saturable transition between these two molecular states is induced by light [26] [27].

\section{Stochastic Switching and Readout}

The diffraction limit does not prevent the nanometer-precise localization of a single fluorophore, which is nowadays a standard tool in single molecule experiments [28]. It does prevent, though, discerning two molecules within the diffraction limit $d$, unless they have distinct spectral properties.

Therefore, an alternate possibility to obtaining superresolution images would be to label all adjacent molecules in different colors, leading to the case that molecules residing within $d$ are all spectrally distinct and can therefore be distinguished just by their emission properties. However, as such a labeling is not feasible, photoswitching is used. If it is ensured that within $d$, only a single molecule is in the bright (on) state and all others are in the dark (off) state, the position of this single emitter can be precisely localized by centroid calculation. Therefore, a sparse population of the on-state has to be ensured. The precision of the localization depends on the number $m$ of emitted photons and is given by $\lambda /[2 n \sin \alpha \sqrt{m}]$ [29]. By subsequential stochastic switching of the fluorophores, detection of $m$ photons and calculation of the position within the sample, a superresolution image can be reconstructed.

This concept is used in several methods like PALM [30] [31], STORM [32] and GSDIM (Ground State Depletion with Individual Molecule return) [33], which differ in the molecular states used for switching. 


\subsection{Labeling Methods for Fluorescence Imaging}

First and also very fundamental step in fluorescence experiments is the labeling of the sample with a fluorescent probe. For many applications even more crucial than the choice of the fluorophore is the method by which the fluorophore is specifically attached to the structure under investigation.

Several techniques for this specific targeting exist, which will be presented in the following paragraphs.

\subsubsection{Immunocytochemistry}

A widespread method for labeling proteins in cells is immunolabeling. It exploits the specific binding of antibodies to antigens in biological tissue [34].

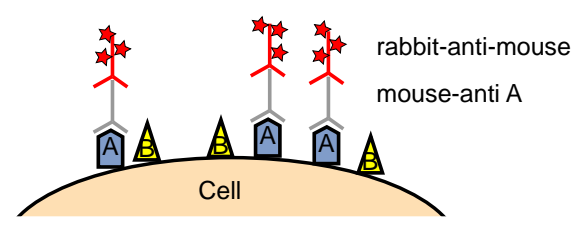

Figure 1.3: Principle of immunocytochemistry: An antibody (mouse-anti-A) specificto the protein of interest (antigen A) recognizes and binds to A. After a washing step, a second antibody (rabbitanti-mouse) against the first antibody is added, which is decorated with the fluorescent dye. It recognizes and binds to the first antibody, which leads to the labeling of the protein of interest with the fluorophore.

For this purpose, the cells are incubated with an antibody recognizing the protein of interest, the antigen. Either this antibody carries the fluorophore, which leads to the direct attachment of the fluorophore to the protein (direct labeling), or a so-called secondary antibody decorated with the fluorophore is added, which recognizes and binds the first antibody (indirect labeling). With the latter method, there is a multiplicative effect, because often more than one secondary antibody binds to the primary antibody; also, mostly, the antibody carries more than one fluorophore. Therefore, very bright samples can be obtained. Because of this feature and the free choice of the fluorophore, immunocytochemistry has become the gold standard for STED imaging of cells and tissue.

The drawback of this method, however, is that in most cases, the cells have to be fixed and permeabilized to ensure that the antibodies reach the antigen. Therefore, live cell imaging is not generally possible, which leads to a severe confinement of the possible applications of STED microscopy in biology.

For conventional fluorescence microscopy, however, other methods are well-established which do not suffer from these constraints. 


\subsubsection{Autofluorescent Proteins}

A milestone for fluorescence microscopy was the discovery, isolation, cloning and improvement of green fluorescent protein (GFP), which was rewarded with the 2008 Nobel Prize in chemistry to Osamu Shimomura, Martin Chalfie and Roger Tsien. Upon irradiation with blue light, green fluorescence is emitted by this protein. Nowadays, improved variants of GFP are a daily tool in the life sciences, where they are used as fusion proteins to monitor expression, structure and activity of all kinds of proteins within living systems [35].
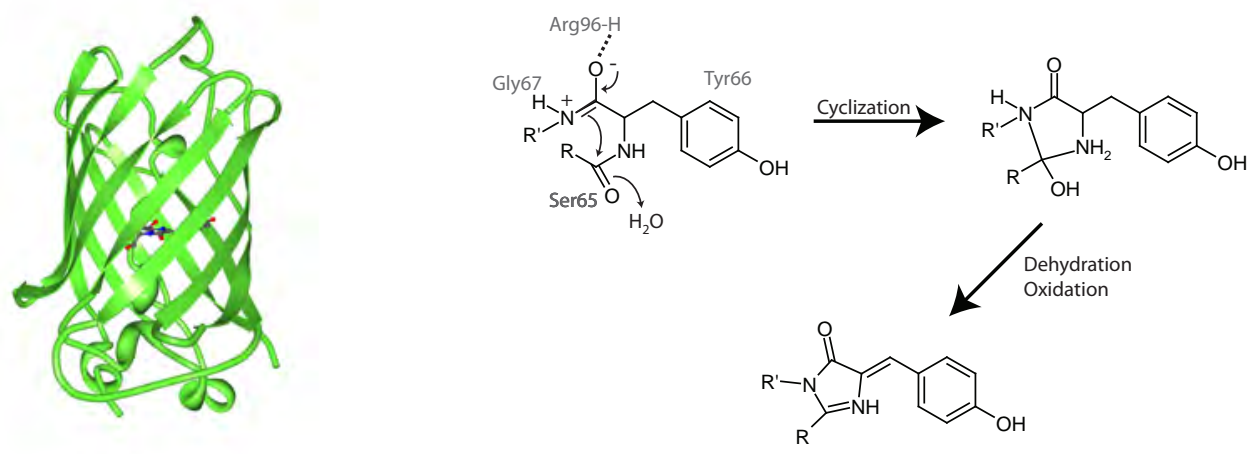

Figure 1.4: The green fluorescent protein. The chromophore is surrounded by a barrel-like structure (left). Steps involved in the autocatalytic formation of the chromophore (right).

Isolated in 1962 by O. Shimomura and colleagues from the jellyfish Aequorea victoria, it took 30 years until D. Prasher and M. Chalfie reported the successful cloning and expression of GFP in various organisms. The structure of GFP was solved soon after in 1996 in the lab of R. Tsien and S. Remington [36]: It is a barrel-shaped protein, where 11 beta-sheets form a cylinder around the chromophore, which is autocatalytically formed via a cyclization of three amino acids of the primary amino acid sequence. Against earlier hypotheses, the formation of the chromophore does not depend on additional cofactors or enzymes; only the presence of oxygen is required. Depending on the exact structure of the chromophore and the direct environment, different color variations have recently been developed, spanning the whole visible spectrum from blue (blue fluorescent protein, BFP), over green and yellow (yellow fluorescent protein, YFP) to far-red (DsRed, mPlum). Also, various sensors like calciumsensitive GFP-variants have been created [37].

These developments made GFP-like proteins an indispensable tool in the modern life sciences.

However, although a lot of effort has been invested in improving the properties of GFPlike proteins, there still are some drawbacks. Examples include the rather large size of the protein $(4.2 \mathrm{~nm} \times 2.4 \mathrm{~nm} \times 2.4 \mathrm{~nm})$, aggregation tendencies and the limited photostability. Therefore, the quest for different labeling approaches still continues. 


\subsubsection{Selflabeling Protein Tags}

Obviously, organic fluorophores offer several advantages when compared to genetically encoded ones, as discussed above. Probably most importantly, there are no restrictions regarding the structure of the chromophore, and complex modifications are easily introduced. However, for live cell imaging, an adequate method for selective labeling of the structure of interest is necessary.

For several structures, a specific labeling method exists which utilizes a certain affinity of the protein of interest. Examples include the labeling of receptors by incubation with the ligand carrying the desired fluorophore, or utilization of the high affinity of phalloidin to actin. However, this works only for some specific proteins and is not generally extendable.

Recently, a new class of genetically encoded tags have been developed, which bind modified dyes via covalent or non-covalent interaction [38]. When these tagging proteins are fused to the protein of interest, incubation of the cells expressing the fusion protein with the substrate-bound dye leads to a specific labeling of the desired structure (see figure 1.5).

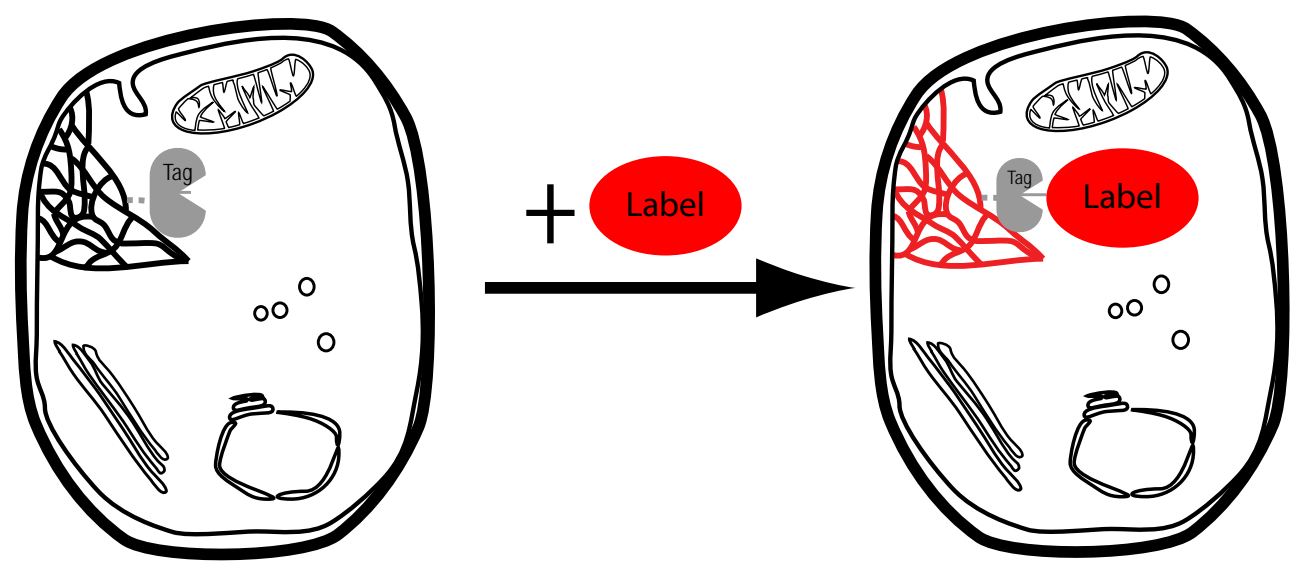

Figure 1.5: Scheme of a live cell labeling approach using organic dyes: a cell expressing a tagging protein fused to the structure of interest is incubated with a modified dye, which is recognized and bound by the tagging protein.

The specific recognition and binding of the modified fluorophore can be due to different effects. Either the binding is covalent or non-covalent; also, the binding itself can be direct or enzyme-mediated. Typical representatives for each of the cases are shown in figure 1.6 and explained in the following paragraphs. 
A

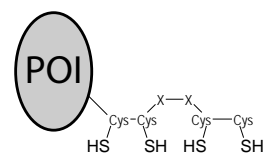

$+$
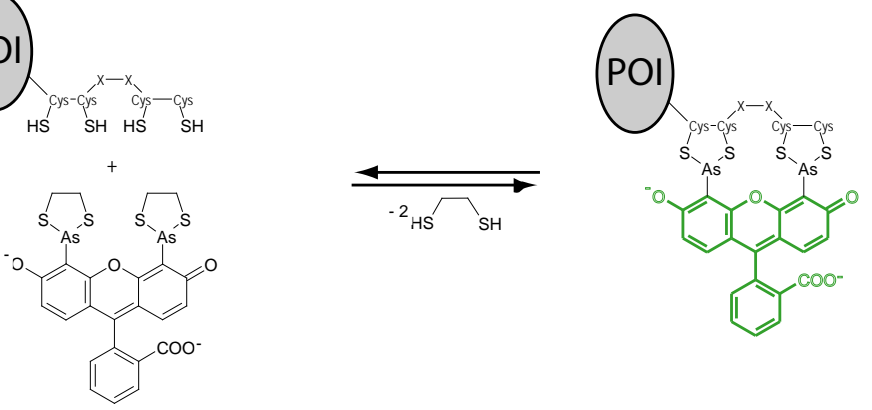

B
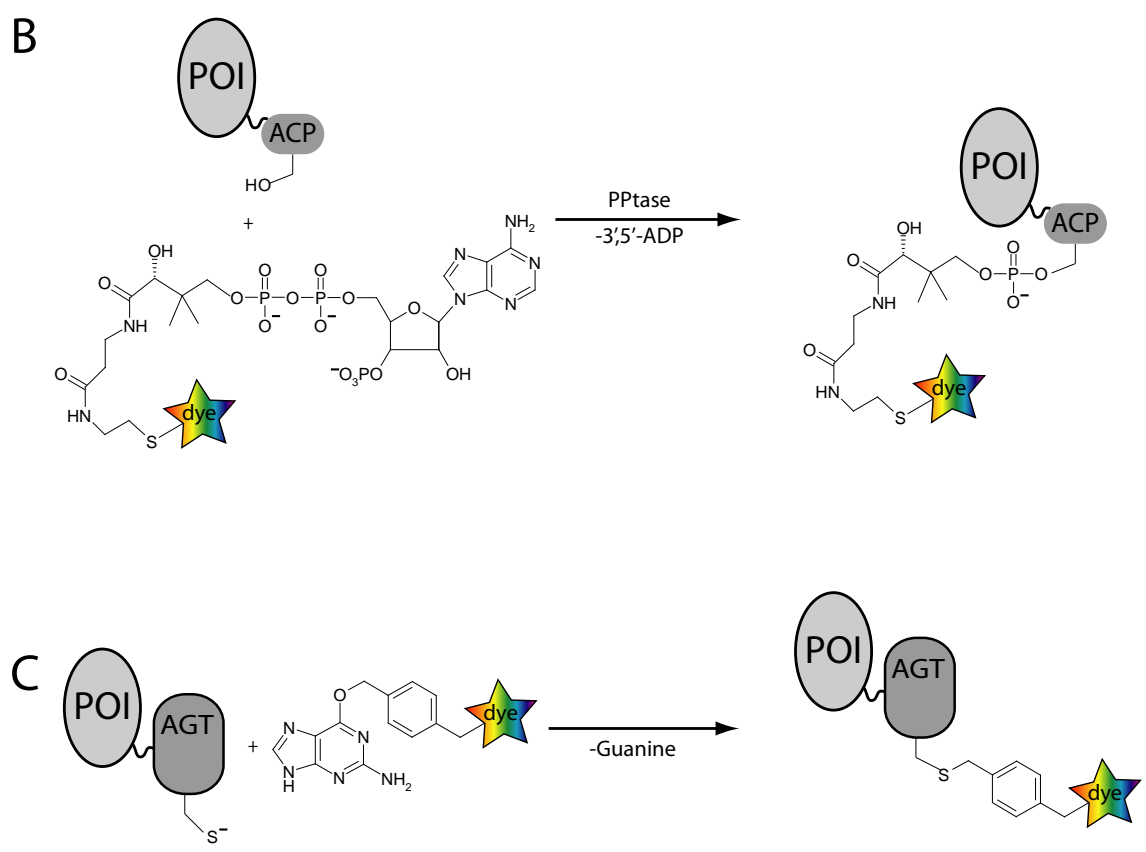

Figure 1.6: Different tagging approaches based on using fusion proteins. A: Binding of a biarsenical-modified fluorescein to the protein of interest (POI) via electrostatic interaction with a tetracysteine-motif. Fluorescence only occurs in the complexed state, which leads to lower background. B: Enzyme-mediated covalent attachment of coenzyme A derivatives to Acyl Carrier Protein (ACP). C: Selflabeling of human alkylguanine DNA alkyl transferase (hAGT) with modified benzylguanines. In the latter two examples, basically any dye can be attached to the protein of interest within living samples. 


\section{Non-covalent Interaction}

A very elegant approach for attaching organic dyes to specific proteins in living cells utilizes the affinity of arsenoxides to closely spaced cysteine pairs [39] (figure 1.6A). Two arsenoxide groups were introduced into fluorescein, forming $\mathrm{FlAsH}$, which binds with high affinity to the peptide sequence CCXXCC, where $\mathrm{C}$ stands for cysteine and $\mathrm{X}$ can be any amino acid. If this peptide sequence is genetically attached to the protein of interest, incubation with the cell permeable FlAsH leads to specific labeling. Recent work has shown that the affinity can be increased by using proline and glycine between the cysteine residues, which helps to get a brighter signal [40]. The tetracysteine-motif is hardly found in cellular proteins, enabeling the specific targeting of the fluorophore to the protein of interest. Importantly, FlAsH itself is non-fluorescent; fluroescence occurs only upon binding to the tetracysteine motif. This feature decreases background originating from unbound dye. Another advantage is the small size of the label; at best, only 12 amino acids plus the organic fluorophore are attached to the protein of interest. The strongly reduced size compared to GFP (238 amino acids) can be crucial for the succesful tagging of sterically demanding proteins.

However, the synthesis of biarsenic compounds is rather tedious work. Up to now, only few derivatives have been synthesized. The existing ones (FlasH and ReASH and similar molecules) posses a rather low photostability even under widefield illumination and are therefore generally not well suited for STED microscopy. With the development of different and especially more photostable fluorophores, which would also be very welcome for conventional fluorescence microscopy, this approach will certainly be a versatile addendum for live cell high resolution microscopy.

Similar approaches using non-covalent interactions exist which also allow for the usage of different fluorophores; examples include binding to polyhistidine-sequences [41] or to dihydrofolate reductase [42]. However, since the fluorescence is not quenched in the unbound state, extensive washing is required, which also partially removes the ligated fluorophore, leading to low labeling efficiencies.

Therefore, for many cases a covalent binding of the fluorophore to the tagging protein is desired.

\section{Covalent Interaction}

Generally, two possibilities for covalent interaction exist: Either the fluorophore is directly bound by the tagging protein, or the reaction is enzyme-mediated.

An example of the latter method is the labeling with Acyl Carrier Protein (ACP) using derivatives of coenzyme A [43] (figure 1.6B): Here, the enzyme pptase (phosphopantetheine transferase) transfers a 4-phosphopantetheine conjugated dye from coenzyme A to a serine residue of the ACP-tag. The main advantage is that the ACP-tag is relatively small, only 77 amino-acids; there is even potential of further decreasing the size of this tag. However, the coenzyme A derivatives are not membrane-permeable. Therefore, this approach is limited to the cell surface, unless the cell is subjected to microinjection or other disturbances of the intact membrane. 
A protein tag which is useful for intracellular application as well is human alkylguanine DNA alkyl transferase (hAGT; figure 1.6C) [44] [45]. It is derived from a DNA repair protein, which removes alkylgroups from alkylated $\mathrm{O}^{6}$-guanine nucleotides by transferring them to a reactive cysteine. This reaction is not limited to alkylated DNA, but can be extended to free benzylguanines and related structures carrying different substituents at the 4-position of the benzyl ring. Upon incubation with the substrate, the nucleotide-moiety is recognized by hAGT and a covalent bond is formed between the protein and the dye, leading to a specific labeling of the protein of interest. In principle, any organic dye can be used as a substituent, leading to a broad variety of substrates for hAGT. Nonetheless, for labeling of intracellular structures, membrane permeable substrates have to be used, which narrows down the fluorophore availability.

\subsection{Objective of This Work}

The objective of this work was to establish live cell STED microscopy. So far, live cell STED imaging was restricted to specific applications [20] [23] and not generally extendable to examine arbitrary structures.

Therefore, labeling approaches compatible with live cell experiments were analyzed for their performance in STED microscopy. By using a yellow fluorescent protein, a lateral resolution down to $50 \mathrm{~nm}$ was achieved within living mammalian cells. Also, short movies were recorded, watching structural changes on the nanoscale. Not only lateral resolution enhancement, but also axial enhancement by a factor of 3 compared to confocal imaging was achieved within living cells. Usage of fluorescent proteins also allows for the subdiffraction imaging of yeast, as was demonstrated by imaging protein clusters on the cell membrane with a lateral resolution of about $50 \mathrm{~nm}$. By using genetically encoded tagging proteins binding organic dyes, $40 \mathrm{~nm}$ resolution was achieved using TMR as a fluorophore in living mammalian cells. This labeling approach was used for taking nanoscopic videos as well.

The results presented here broaden the possible application range of STED microscopy, facilitating STED imaging using genetically encoded tags. This also finally allows for leveraging the main advantage of light nanoscopy compared to other methods offering a similar or even better resolution: the possibility to examine living specimen. 


\section{Techniques}

The theoretical basis for achieving superresolution with optical microscopy, as well as possible labeling methods for live cell imaging have been explained in the previous chapter. This chapter describes the practical implementation and the sample preparation used in this work for live cell STED microscopy.

\subsection{STED Microscopy}

As explained in the introduction, stimulated emission depletion is a versatile tool for achieving subdiffraction resolution. In this work, a STED microscope which features a tunable STED laser and various excitation lasers has been built and employed, offering the opportunity to study different fluorophores across the whole visible spectrum. The setup and its characteristics are introduced in the following paragraphs.

\subsubsection{The Microscope}

The heart of a STED microscope is the STED beam, which is overlaid onto the excitation beam. Since the STED beam is shaped in a way that it offers a central zero intensity, deexcitation only takes place in the outer part of the excitation spot, leading to a confinement of fluorescence to the very center of the excitation spot, thereby squeezing it down to subdiffraction size. Conveniently, the setup is based on a confocal microscope. A scheme is shown in figure 2.1

For fluorescence excitation, laser diodes emitting 100 ps pulses at $490 \mathrm{~nm}$ or at $532 \mathrm{~nm}$ (depending on the fluorophore used; both PicoTA, Picoquant, Germany) were used. STED light was provided by an optical parametric oscillator (OPO, APE, Berlin, Germany) which was synchronously pumped by a mode-locked Ti:Sapphire laser (MaiTai, Spectra Physics, Mountain View, CA) operating at a repetition rate of $80 \mathrm{MHz}$. The wavelength of the converted light can be adjusted between 550 and $670 \mathrm{~nm}$. The fs-pulses were stretched to 150$250 \mathrm{ps}$ by dispersion in a polarization-preserving fiber $(80 \mathrm{~m}$, cut-off at $633 \mathrm{~nm}, \mathrm{OZ}$ optics, Ottawa, Canada). To avoid non-linear optical effects in the fiber, pulses were pre-stretched by passing them through $30 \mathrm{~cm}$ of $\mathrm{SF}_{6}$ glass rods before coupling into the fiber. Correct timing of excitation- and STED pulses was ensured by triggering the excitation diode with a signal from the Ti:Sapphire laser via a variable electronic delay box (home-built). Shaping the STED beam to feature the central zero intensity was accomplished by using a phaseplate, which partially adds a phase retardation to the STED beam by passage through an optically denser material (homebuilt or RPC photonics, Rochester, NY, USA). 


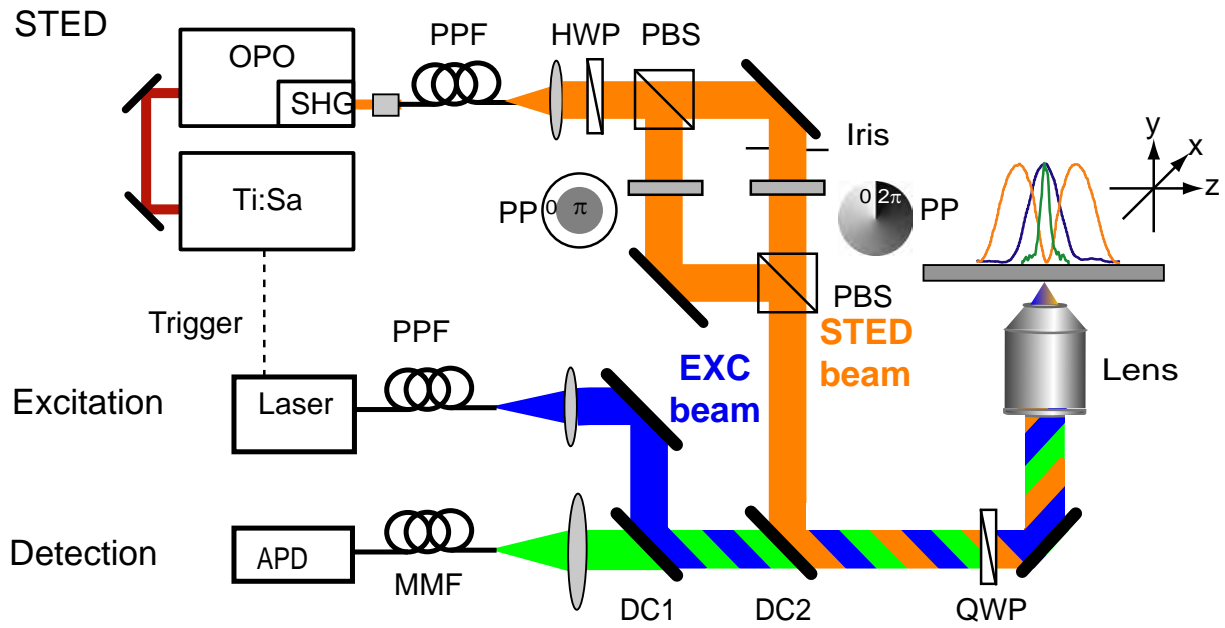

Figure 2.1: Scheme of the STED microscope used. APD, avalanche photo diode; DC, dichroic mirror; HWP, half-wave plate; MMF, multimode optical fiber; OPO, optical parametric oscillator; $\mathrm{PP}$, phase plate; PBS, polarizing beam splitter cube; PPF, polarization preserving optical fiber; QWP, quarter-wave plate; SHG, second-harmonic generation; Ti:Sa, Ti:sapphire laser.

Both the excitation (Exc) and the STED beams were coupled into a 1.4 numerical aperture oil immersion lens (HPC PL APO, 100x, Leica Microsystems, Mannheim, Germany) using two custom-made dichroic beamsplitters DC1 (DC488 or DC532, Chroma Technology, Rockingham, VT, USA) and DC2 (HR620 HT UV for STED at $590 \mathrm{~nm}$ and HR660 HT UV for STED at 650 - $660 \mathrm{~nm}$, custom made, Laser Components GmbH, Olching, Germany). For other wavelength settings, the mirrors were accordingly chosen. An achromatic quarterwave plate (QWP 460-680 nm, B. Halle, Berlin, Germany) converts the linearly polarized light into circularly polarized light in front of the objective lens. The emitted fluorescence was collected by the same lens as was used for focusing into the sample and directed onto a multimode fiber $(\mathrm{d}=62.5 \mu \mathrm{m})$ which served as the confocal pinhole; the opening diameter corresponds to $78 \%$ of the Airy disc of the excitation light. The fluorescence signal was detected by an avalanche photodiode (SPCM-AQR-13-FC, PerkinElmer, OptoElectronics Europe, Germany) operating in the counting mode. The image was obtained by scanning the sample with a piezo-stage (Melles Griot, UK) at a typical pixel dwell time of $0.05 \mathrm{~ms}$ and a pixel size of $20 \mathrm{~nm}$ x $20 \mathrm{~nm}$ or $15 \mathrm{~nm}$ x $15 \mathrm{~nm}$.

Alternatively, a beamscanning setup was employed: overlaid excitation- and STED beam were reflected off a $16 \mathrm{kHz}$ resonant mirror (SC-30, EOPC, Glendale, NY, USA) for one axis beam scanning. A piezo stage (733-P3DD and E-710, Physik Instrumente, Karlsruhe, Germany) was used for scanning along the second lateral axis. The resonant scanning mirror was imaged onto the back aperture of the objective lens via a tube and a scanning lens matching the objective lens (Leica). Here, dwelltimes typically between 5 and $20 \mu$ s were used; however, the setup also allows for fast scanning in the sub- $\mu$ s dwelltime regime. 


\subsubsection{Shaping the Effective Spot of Excitation}

Depending on the phasemask used, different focal shapes of the STED beam can be obtained. A helical phaseramp causing a phaseshift from 0 to $2 \pi$ leads to a doughnut-shaped beam in the focal plane, which is used for increasing the resolution in the lateral direction $\left(\mathrm{STED}_{\mathrm{xy}}\right.$, see figure 2.2. . In a different geometry, a constant phaseshift of $\pi$ is introduced over a central circular area. This generates a focal intensity shape where the main intensities are below and above the focal plane, leading to a deexcitation and therefore resolution enhancement mainly along the z-axis (figure 2.2, $\mathrm{STED}_{\mathrm{Z}}$ ). Depending on the structure to be imaged, either one of them or a combination of both can be used. The phaseshift is realized by adding a transparent material (here: a polymer) with a refractive index $n>1$ to a glass substrate. The thickness of the material has to be adjusted depending on the wavelength used.

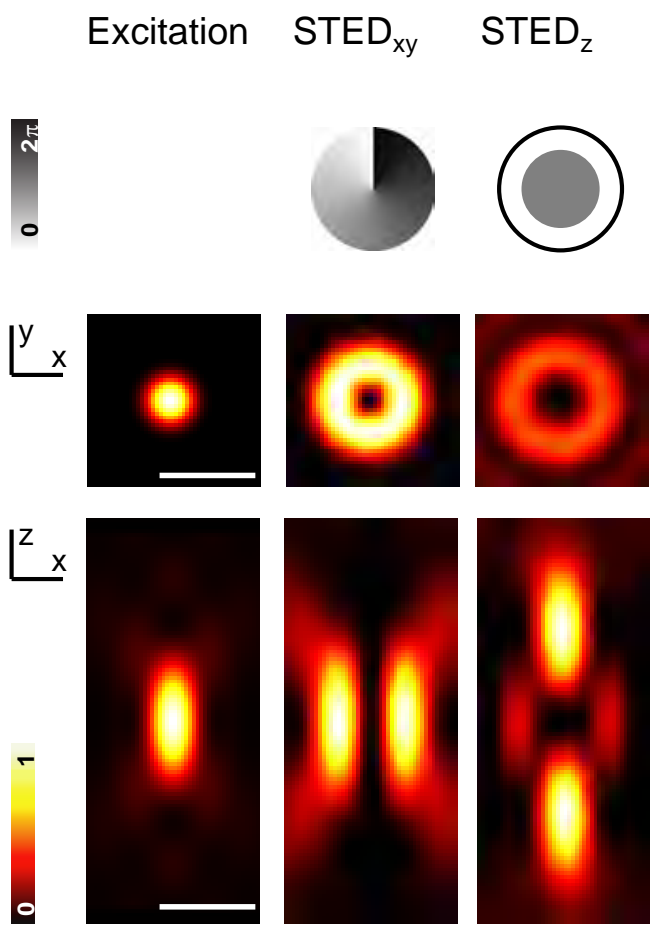

Figure 2.2: Generation of different point spread functions used for STED imaging. Left: xy- and xz- view of a regularly focused beam, as used for the excitation in STED microscopy. Center: Introduction of a helical phaseramp from 0 to $2 \pi$ (top) leads to a doughnut-shaped focus in the lateral direction $\left(\mathrm{STED}_{\mathrm{xy}}\right)$. Right: A circular phasepattern is used for generating resolution enhancement also along the optical axis $\left(\mathrm{STED}_{\mathrm{Z}}\right)$. The $\mathrm{xz}$-section shows that in this case the main intensities are located along the z-axis upon focusing, featuring a central zero. All spots represent data calculated by using experimental conditions for excitation at $488 \mathrm{~nm}$ and STED at $595 \mathrm{~nm}$. Scale bars $500 \mathrm{~nm}$. 


\subsubsection{Performance of the STED Microscope}

The superior imaging capability of the STED microscope compared to a conventional confocal microscope is demonstrated in figure 2.3. Fluorescent beads with a diameter of $23 \mathrm{~nm}$ were imaged in the STED mode (A, right) and as a comparison in the confocal way by blocking the STED beam (A, left).

To estimate the resolution, a depletion curve (figure 2.3 B) was recorded. To achieve this, a regularly focused STED beam (e.g. without a phasemask) was overlaid onto the excitation beam. For different STED intensities, the residual fluorescence was measured. The STED depletion coefficient $\eta$ was calculated by dividing the residual fluorescence for each STED intensity by the total fluorescence without STED.

The higher the applied STED intensity, the better the resolution (figure 2.3 C). In addition to the experimental images, the respective effective PSF for each STED intensity was also calculated (figure $2.3 \mathrm{C}$, right part), showing the effect of increasing STED intensity on the resolution obtainable. 
A
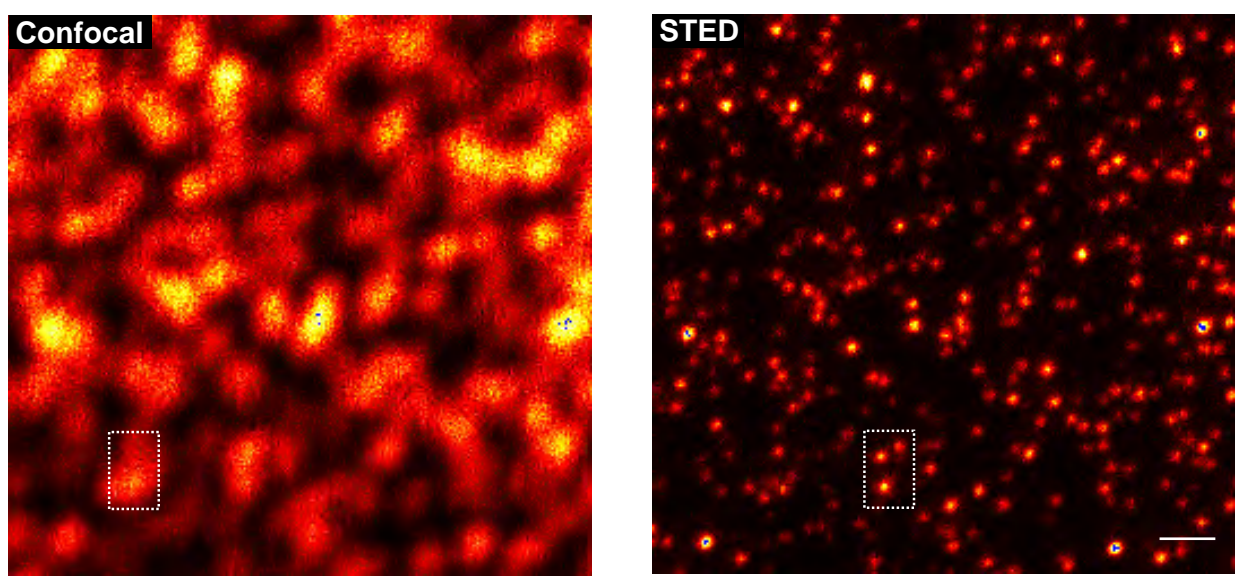

B

C
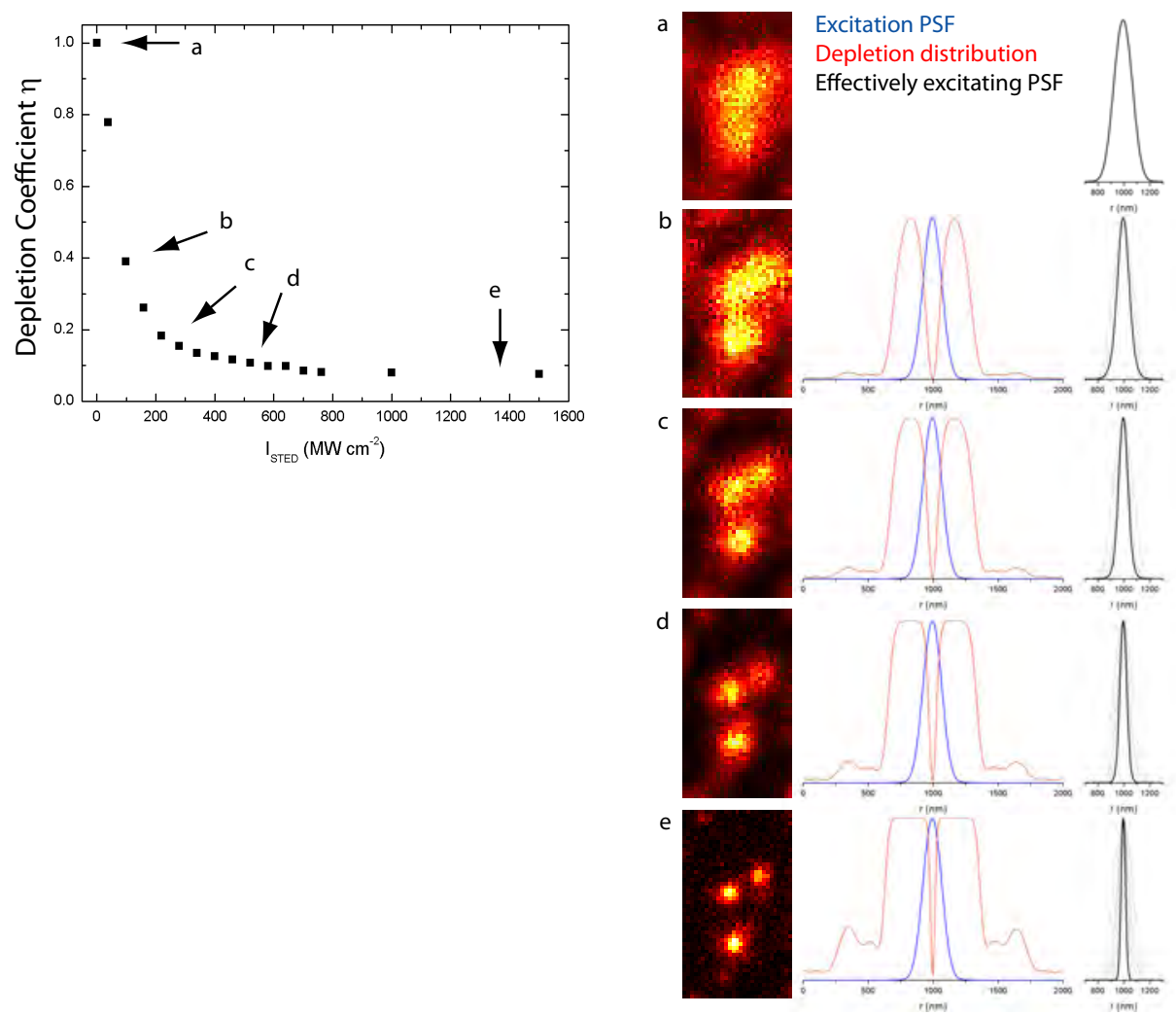

Figure 2.3: Effect of the applied STED intensity on the resolution obtained: Yellow fluorescent beads with a diameter of $23 \mathrm{~nm}$ were imaged under different conditions. A: Comparison between confocal (left) and STED imaging (right) with high STED intensity. B: Depletion curve for yellow fluorescent beads under the conditions applied. C: Both experimental and simulated resolution for different STED intensities indicated in B. Calculated effectively exciting PSFs (black) for different STED intensities were obtained by multiplication of the excitation PSF (blue) with the depletion distribution function (red). Scale bar $500 \mathrm{~nm}$. 


\subsection{Sample Preparation}

For the sample preparation, in most cases, standard biological tools were used; therefore, only a brief description of the conditions and reagents used will be given here. Newly developed or optimized protocols are described in more detail.

\subsubsection{Plasmid Construction}

\section{PCR}

Oligonucleotides used as a primer (Sigma-Aldrich, Steinheim, Germany) were designed to have a length between 25 and 35 nucleotides. PCR (Polymerase Chain Reaction) was performed using Pfu-DNA-polymerase (Fermentas, Burlington, Canada): A reaction volume of $25 \mu \mathrm{l}$ (containing $50 \mathrm{pmol}$ of the priming oligonucleotides, $2 \mathrm{mM}$ of each dNTP, $1 \mathrm{pmol}$ of the respective template DNA and $0.5 \mu \mathrm{l} \mathrm{Pfu}(2.5 \mathrm{U} / \mu \mathrm{l})$ in PCR-buffer (Fermentas); if strong self-annealing of the primer was predicted, $1 \mu \mathrm{l}$ DMSO was added) was subjected to PCR consisting of 30 elongation cycles. The program was composed of an initial 5 minute phase at $95{ }^{\circ} \mathrm{C}$; the cycle [45 seconds at $95{ }^{\circ} \mathrm{C}$; 60 seconds $50{ }^{\circ} \mathrm{C}-65{ }^{\circ} \mathrm{C} ; 5$ minutes at $72{ }^{\circ} \mathrm{C}$ ] was repeated for 30 times; complete annealing was ensured by a final phase of 10 minutes at $72{ }^{\circ} \mathrm{C}$.

\section{Digestion and Ligation}

Digestion using restriction enzymes (NEB or Fermentas) was performed over night at $30{ }^{\circ} \mathrm{C}$ in the appropriate manufacturers buffer. For dephosphorylation, linearized plasmids were incubated with calf intestine phosphatase (NEB) for 60 minutes at $30{ }^{\circ} \mathrm{C}$. Electrophoresis with $1 \%$ agarose gels was employed for size separation; DNA extraction was performed using a DNA gel extraction kit (Qiagen, Hilden, Germany).

For ligation, $100 \mathrm{fmol}$ linearized plasmid-DNA and $300 \mathrm{fmol}$ insert were incubated with T4-DNA-ligase (Fermentas) for 4 hours at room temperature. After a 10 minute long heat inactivation of the ligase at $65{ }^{\circ} \mathrm{C}$, the DNA was transformed using electroporation into electrocompetent $E$. coli, which were grown on lysogeny-broth (LB) agar plates carrying the appropriate resistance. Positive clones were picked; the DNA was amplified in E. coli growing for 12 hours in LB medium at $37{ }^{\circ} \mathrm{C}$ in a shaking incubator. DNA purification relied on alkaline hydrolysis; DNA purification kits (Qiagen) were used according to the manufacturer's manual.

\section{Plasmid Design}

For expression of the different fluorescent proteins in E. coli, the respective coding DNA sequence was introduced into the vector pQe30, pQe31 or pQE32 (Qiagen), which are prokaryotic expression vectors for high expression of his-tagged proteins in E. coli under the control of the Lac-operon.

For targeting fluorescent proteins at the ER, the vector $\mathrm{pEF} / \mathrm{myc} / \mathrm{ER}$ (Invitrogen, Carlsbad, CA, USA) was used. Coding sequences for the fluorescent proteins were introduced as 
described above. Tubulin-fusion proteins were created by exchange of the coding sequence of the fluorescence protein in pEGFP-Tub (Clontech Laboratories, Mountain View, CA, USA). GPI-anchored proteins are based on the vector pAEMXT-ACPwt-GPI (Covalys Biosciences, Witterswil, Switzerland). All other fusion proteins were constructed by Gateway vector conversion [46] (Invitrogen) using the empty destination vector pSEMS-GatewaySNAP26m or pSEMS-SNAP26m-Gateway (Covalys) for hAGT fusion proteins. For tagging with ACP, HaloTag or autofluorescent proteins, the coding sequence of hAGT was substituted with the coding sequence for the accordant protein. Destination vectors were pDONR223-MAP2, pDONR223-CAV1, pDONR223-GJA1, pDONR223-GJA4 and pDONR223-VIM.

\subsubsection{Biological Systems used in this Work}

\section{E. coli}

DNA replication as well as protein overexpression were performed using electrocompetent E. coli, which were grown at $37^{\circ} \mathrm{C}$ in lysogeny-broth (LB) medium $(5 \mathrm{~g} / 1$ yeast extract, $5 \mathrm{~g} / 1$ $\mathrm{NaCl}, 10 \mathrm{~g} / \mathrm{l}$ tryptone, $5 \mathrm{ml} / 11 \mathrm{~N} \mathrm{NaOH}$ ). Antibiotics were added depending on the plasmid carried.

For DNA replication, the strain DH5 $\alpha$ was grown in $200 \mathrm{ml} \mathrm{LB}$ medium in a shaking incubator for 12 hours, before harvesting the bacteria by centrifugation. For the screening experiments, the strain HMS transformed with pQe-plasmids carrying the respective fluorescent proteins was grown in the same way; upon reaching an OD600 of 0.6, expression was induced by adding $1 \mathrm{mM}$ isopropyl- $\beta$-thiogalactosid (IPTG, AppliChem, Darmstadt, Germany). After 4 hours of growth at $30{ }^{\circ} \mathrm{C}$, cells were harvested by centrifugation and fixed by 1 of hour incubation in $4 \%$ freshly prepared paraformaldehyde (Sigma-Aldrich); after washing with PBS (phosphate buffered saline, Sigma-Aldrich), they were mounted on coverslips using mowiol (6 g glycerol (Merck, Germany), $2.4 \mathrm{~g}$ mowiol 4-88 (Hoechst, Germany), $6 \mathrm{ml}$ water, $12 \mathrm{ml} 0.2 \mathrm{M}$ Tris ph 7.2 buffer) as embedding medium.

\section{Yeast}

Fluorescently labeled yeast cells were prepared by C. Wurm (MPI for Biophysical Chemistry, Göttingen, Germany), details can be found in the literature [47]. For imaging, yeast growing on agar plates for 24 hours at $30{ }^{\circ} \mathrm{C}$ were scraped from the plate, washed with PBS and embedded in $1 \%$ low-melting agarose immediately before imaging.

\section{Mammalian Cells}

\section{Cell Culture}

Mammalian PtK2 (Potorous tridactylus kidney) cells were grown in $25 \mathrm{ml}$ flasks at $37{ }^{\circ} \mathrm{C}$ and $7 \% \mathrm{CO}_{2}$ in DMEM (Dulbeccos Modified Eagle Medium, Invitrogen) containing $4.5 \mathrm{~g} / \mathrm{l}$ glucose, which was supplemented with $10 \%$ fetal bovine serum (Invitrogen), $1 \mathrm{mM}$ sodiumpyruvate (Sigma Aldrich), $100 \mathrm{u} / \mathrm{ml}$ penicillin and $50 \mu \mathrm{g} / \mathrm{ml}$ streptomycin (BioChrom, Berlin, 
Germany). The same conditions were applied for growth of rat phaeochromozytom PC12 cells and cervical cancer HeLa cells.

For imaging, cells were seeded on round $10 \mathrm{~mm}$ diameter coverslips.

\section{Transfection}

Upon reaching $80 \%$ confluence, which was about 24 hours after seeding, cells were transfected with endotoxin-free DNA by using Nanofectin (PAA, Pasching, Austria) according to the protocol. The cells were incubated for at least $24 \mathrm{~h}$ before imaging.

\section{Imaging Conditions}

Mammalian cells were imaged in a homebuilt chamber to which the coverslips were transferred immediately before imaging. DMEM containing no phenolred supplied with $10 \mathrm{mM}$ HEPES (4-(2-hydroxyethyl)-1-piperazineethanesulfonic acid, Sigma-Aldrich) for maintaining physiological $\mathrm{pH}$ was used as imaging medium. The chamber allows for temperature control, however, most experiments were performed at room temperature.

\subsubsection{Organic Dyes as a Live-Cell Compatible Fluorescent Label Labeling of Tagging Proteins with Organic Dyes}

Labeling hAGT-expressing cells was performed by incubation of the cells in a solution of the substrate in DMEM at $37{ }^{\circ} \mathrm{C}$ and $5 \% \mathrm{CO}_{2}$. For labeling with CP-TMR as a substrate, 15 minutes incubation in a $1 \mu \mathrm{M}$ solution was performed. For all other substrates, cells were incubated in a $5 \mu \mathrm{M}$ solution for 30 minutes. Afterwards, cells were washed with fresh DMEM for 30 min under the same conditions before imaging.

For ACP-labeling, cells were incubated for 30 min in DMEM supplemented with $10 \mathrm{mM}$ $\mathrm{MgCl}_{2}, 5 \mu \mathrm{M}$ substrate and $1 \mu \mathrm{M}$ pptase (Covalys Biosciences).

\section{Synthesis of Substrates for hAGT and ACP}

For the synthesis of the substrates for hAGT, NHS-ester derivatives of the respective dyes were reacted with the aminogroup of $\mathrm{O}^{6}$-benzylguanine (BG) or 4-(4-/aminomethyl)benzyloxy)-6-chloropyrimidine-2-amine (CP). Unless otherwise noted, all chemicals were purchased from Sigma-Aldrich.

$100 \mu \mathrm{g}$ NHS-modified dye (Atto-Tec, Siegen, Germany) in $15 \mu \mathrm{l}$ DMF were added to $100 \mu \mathrm{g} \mathrm{BG}-\mathrm{NH}_{2}$ or $\mathrm{CP}-\mathrm{NH}_{2}$ (both Covalys) in $15 \mu \mathrm{l}$ DMF containing $0.1 \mu \mathrm{l}$ triethlyamine; the reaction was carried out at room temperature over night. The crude mixture was purified via reverse phase preparative HPLC (eluent: water/acetonitrile).

Coenzyme A derivatives were synthesized using maleimide derivatives of the fluorophores, which react with the thiolgroup contained in coenzyme A. The coupling reaction for different thiol-reactive dyes was carried out by AttoTec or V. Belov (MPI for Biophysical Chemistry, Göttingen, Germany). 


\section{Organic Dyes Used for Labeling}

Organic dyes in different wavelength regimes were used in this work for labeling both with ACP and with hAGT. For ACP-tagging, coenzyme A derivatives were synthesized as described above, employing dyes covering the visible and near-infrared regime. For hAGTlabeling, both $\mathrm{CP}$ and $\mathrm{BG}$ derivatives were prepared and the membrane permeability was tested. To find suitable membrane-permeant substrates, several dyes in the same spectral region were used. Table 2.1 itemizes the substrates examined in this work; excitation- and STED wavelength used, as well as the membrane permeability of the hAGT substrates are indicated.

\begin{tabular}{|c|c|c|c|c|c|}
\hline Fluorophore & $\lambda_{\text {Exc }}(\mathrm{nm})$ & $\lambda_{\text {STED }}(\mathrm{nm})$ & ACP & hAGT & Permeability \\
\hline \hline Atto488 & 488 & 595 & $/$ & $\checkmark$ & no \\
\hline Oregon Green & 488 & 595 & $/$ & $\checkmark$ & yes $^{\ddagger}$ \\
\hline DY-505 & 488 & & $/$ & $\checkmark$ & yes $^{\ddagger}$ \\
\hline Atto532 & 488 & 612 & $\checkmark$ & $\checkmark$ & no \\
\hline DY-547 & 532 & & $/$ & $\checkmark$ & no \\
\hline Atto550 & 532 & 650 & $\checkmark$ & $\checkmark$ & no \\
\hline TMR & 532 & 650 & $/$ & $\checkmark$ & yes* \\
\hline Atto565 & 532 & 660 & $\checkmark$ & $\checkmark$ & no \\
\hline DY-647 & 633 & 770 & $/$ & $\checkmark$ & no \\
\hline Alexa647 & 633 & 770 & $\checkmark$ & $\checkmark$ & no \\
\hline Atto647N & 633 & 770 & $\checkmark$ & $\checkmark$ & no \\
\hline KK & 633 & 770 & $/$ & $\checkmark$ & no \\
\hline
\end{tabular}

Table 2.1: Organic dyes used as a substrate for ACP- and hAGT labeling. Excitation- and STED wavelengths which were used are itemized. For hAGT-labeling, the membrane permeability of the substrate is also indicated. (*: CP-coupled substrate. ${ }^{\ddagger}$ : BG-coupled substrate. ${ }^{*}$ synthesized by V. Belov, Göttingen; unpublished.) 


\subsection{Screening of Fluorescent Proteins}

To establish live cell STED imaging, fluorescent proteins in different colors were examined. Towards this end, the proteins were overexpressed in E. coli. The performance under STED conditions was tested by applying a regularly focused Gaussian depletion beam, i.e. without the phase-retardation which leads to the doughnut-shaped focal spot. By this means proteins in all spectral ranges were tested. For excitation, an appropriately emitting pulsed laser diode was used; the depletion wavelength was adapted to the respective spectroscopic properties of the protein by using a tunable OPO.

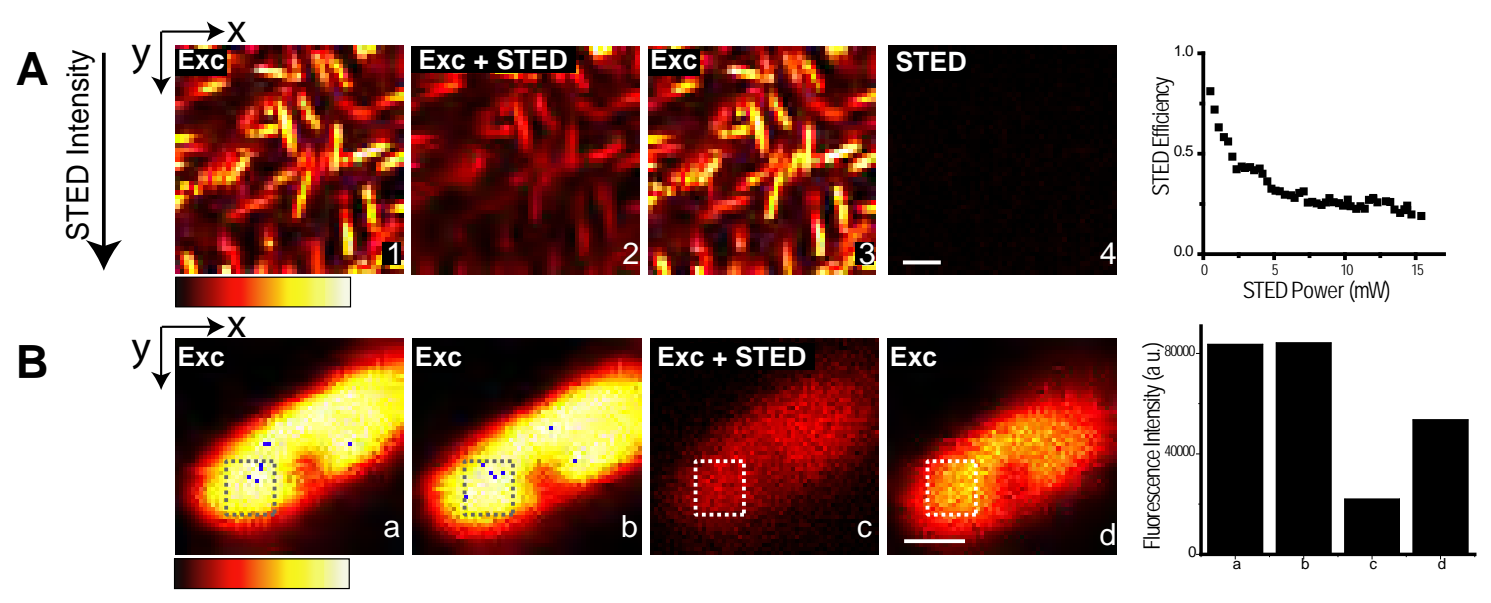

Figure 2.4: Performance of fluorescent proteins under STED conditions. A: Determining the STED efficiency of fluorescent proteins (here: mCherry) expressed in E. coli. Scale bar $2 \mu \mathrm{m}$. B: Bleaching under STED illumination, analyzed in fixed $E$. coli expressing the fluorescent protein mCherry. Scale bar $500 \mathrm{~nm}$.

Figure 2.4 shows an example of the screening experiments: STED efficiencies were determined in $E$. coli by taking xy-images with a large pixelsize to prevent bleaching (A). After taking a conventional confocal image (1), a STED image was recorded with increasing STED power from top to bottom, i.e. along the y-axis (2). To check if bleaching nonetheless occurred, which would tamper with the analysis, an additional confocal image was recorded subsequently(3). Finally, to correct the residual fluorescence intensity under STED illumination for the reexcitation caused by the STED beam, an image with increasing STED intensity where only the STED beam was switched on was taken (4). The fluorescence signal was summed up along the horizontal axis (i.e. with constant STED power). To calculate the STED efficiency, the residual fluorescence intensity under STED illumination was divided by the fluorescence intensity of the confocal image. As the corresponding graph (A, right) shows, the residual fluorescence goes down with increasing STED intensity. In these measurements, however, it does not approach zero, but levels off at a higher value. This is due to the structure of the sample, which shows a substantial extension in all three dimensions: In the outer regions of the STED focus, the intensity decreases, which therefore also 
leads to less efficient suppression of the fluorescence. In an extended sample (as opposed to a pointlike sample), this therefore results in residual fluorescence.

For a given STED intensity, bleaching experiments were performed: The settings were adapted to parameters typically used for imaging (e.g. $30 \mathrm{~nm}$ x $30 \mathrm{~nm}$ pixelsize, $200 \mu \mathrm{s}$ dwelltime). As shown in figure $2.4 \mathrm{~B}$, the fluorescence intensity of a confocal image of fixed E. coli expressing the respective protein before (panel a) and after (panel d) taking a STED image (panel c) was compared. Additionally, a second confocal image was recorded at the very beginning (panel b) to check for excitation bleaching. The signal was summed up within a selected region (white rectangle) and compared, as shown in the histogram in figure 2.4 $\mathrm{B}$. The fraction of residual fluorescence after taking a STED image was then compared for the different proteins.

Since proteins were examined in different spectral ranges, a direct comparison with the same parameters is impossible. The objective of these experiments was to find the best protein for getting images with a maximized resolution and a still adequate signal. Therefore, the parameters had to be adapted to provide the same circumstances under which bleaching can be compared. This especially meant adjusting the STED intensity in a way such that the same resolution gain was generated. Also since, in contrast to the STED wavelength, the excitation wavelength is not tunable in the setup used (because it is less critical), the effective excitation of the fluorophore had to be considered as well.

A compilation of proteins examined within this work is given in table 2.2 .

\begin{tabular}{|c|c|c|c|}
\hline Green & $\begin{array}{l}\text { BFP } \\
\text { EBFP }^{2} \\
\text { Azurite } \\
\text { ECFP } \\
\text { Cerulean } \\
\text { CyPET } \\
\text { Midoriishi-Cyan } \\
\text { AmCyan } \\
\text { EGFP } \\
\text { Monstergreen } \\
\text { AcGFP } \\
\text { GFP } \\
\text { PtGFP } \\
\text { RrGFP } \\
\text { RmGFP } \\
\text { Azami Green }\end{array}$ & Yellow & $\begin{array}{l}\text { EYFP } \\
\text { Venus } \\
\text { Citrine } \\
\text { YPet } \\
\text { PhiYellow } \\
\text { mOrange } \\
\text { mOrange2 } \\
\text { KusabiraOrange } \\
\text { tdTomato } \\
\text { DsRed } \\
\text { TagRFP } \\
\text { mStrawberry } \\
\text { AsRed } \\
\text { Keima } \\
\text { mRFP } \\
\text { mCherry } \\
\text { mPlum }\end{array}$ \\
\hline
\end{tabular}

Table 2.2: Autofluorescent proteins tested within this work for their performance in STED microscopy. 


\section{Live Cell STED Microscopy}

In the recent decades fluorescence microscopy has become an indispensable tool to study biological structures and pathways in living cells, tissues and animals. With the development of superresolution fluorescence imaging such as STED microscopy, the major disadvantage of light microscopy, namely its limited resolution, is eliminated, thereby allowing for subdiffraction imaging. However, so far, most nanoscopic biological studies have been carried out using immunocytochemistry as the labeling technique, which requires in all but some special exceptions the fixation of the cell[48] [49] [50]. Therefore, the possible field of application of superresolution imaging would greatly benefit from a suitable live cell compatible labeling approach.

The creation of fluorescent fusion proteins is a widely used technique for live cell fluorescence microscopy. However, the demands on the photophysical properties of the fluorophore are somewhat higher in STED microscopy. In particular, photostability is a crucial factor.

This chapter shows the successful application of two different labeling techniques based on fusion proteins for STED microscopy: In the first part, autofluorescent proteins are utilized as a fluorophore, whereas in the second part, a fusion protein is used, which covalently binds modified organic fluorophores. Both approaches allow for live cell STED imaging with subdiffraction resolution. This means that a lot of currently ongoing studies of living specimens carried out by conventional microscopy can also be performed with a STED microscope, offering a, thus far, unprecedented spatial resolution. 


\subsection{Live Cell STED Imaging using Fluorescent Proteins}

A lot of effort has been put into the improvement and alteration of Aequorea victoria green fluorescent protein (GFP), leading to a variety of fluorescent proteins in different hues and with different photophysical properties as briefly discussed in chapter 1.4.2. Promising candidates - namely preferentially monomeric, reasonably bright and photostable in confocal microscopy - have been tested in this work for their performance in STED microscopy.

Details about the screening process, as well as a compilation of the proteins tested in this work can be found in chapter 2.3. Briefly, the photostability of proteins overexpressed in $E$. coli under STED illumination was compared; the experimental settings were adapted to the respective photophysical properties. Best results were obtained for enhanced yellow fluorescent protein (EYFP) [36] and Citrine [51] [52], which got its name from its color and pronounced resistance to acidic $\mathrm{pH}$.

A

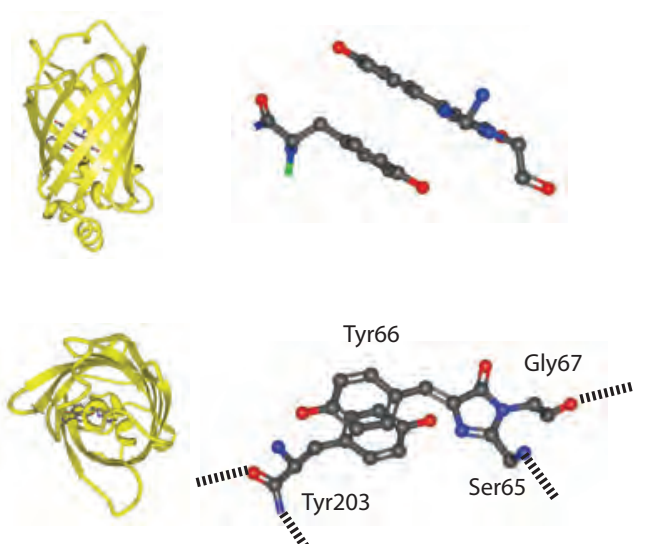

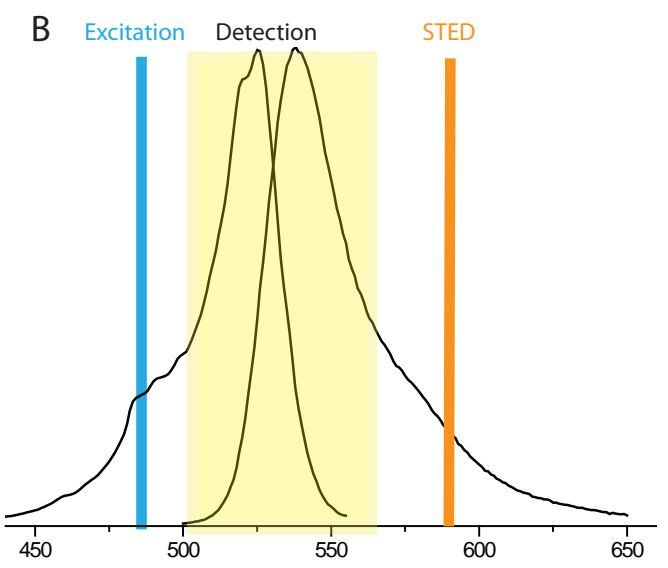

Figure 3.1: Citrine, a yellow fluorescent protein which shows good photostability in STED microscopy. The overall protein structure as well as the chromophore are shown from the side (upper part) and from the top (lower part). The configuration allows for $\pi-\pi$ stacking between the chromophore itself and Tyr203; both show a planar configuration and are aligned parallel to each other. Excitation and STED wavelength as well as the detection window used for nanoscopic imaging are marked in the spectrum (B).

Yellow fluorescent proteins (YFPs) were created by mutating Threonine 203 of GFP to an aromatic amino acids, typically Tyrosine. This residue resides in close proximity to the chromophore. By exchange with an aromatic amino acid, $\pi-\pi$-stacking can occur with the chromophore, extending the delocalized electron system, which is thought to be responsible for the $20 \mathrm{~nm}$ shift to longer excitation and emission wavelengths compared to GFP [35].

The following examples show the successful use of these proteins for live cell nanoscopy using STED. Images were taken using the setup described in chapter 2.1.1. Excitation was performed with a 488-nm laser diode; for depletion, $595 \mathrm{~nm}$ light was employed. Fluorescence was detected in the range between 505 and $565 \mathrm{~nm}$. 


\subsubsection{Live Cell Imaging in Mammalian Cells}

\section{The Endoplasmic Reticulum}

The endoplasmic reticulum (ER) is a eucaryotic cell organelle which forms a network throughout the cell body. Important functions of the ER include the sequestration of calcium as well as the synthesis of macromolecules like steroids or glycogen. Furthermore, it is the location of protein translation, folding and transport; an elaborate quality control system of newly synthesized proteins is also located in this organelle [34].

In the following examples, the fluorescent protein Citrine was localized to the ER by attaching a signal and a retention sequence onto its $\mathrm{N}$ - and $\mathrm{C}$-terminus, respectively, allowing for free diffusion of the fluorescent protein within the ER.
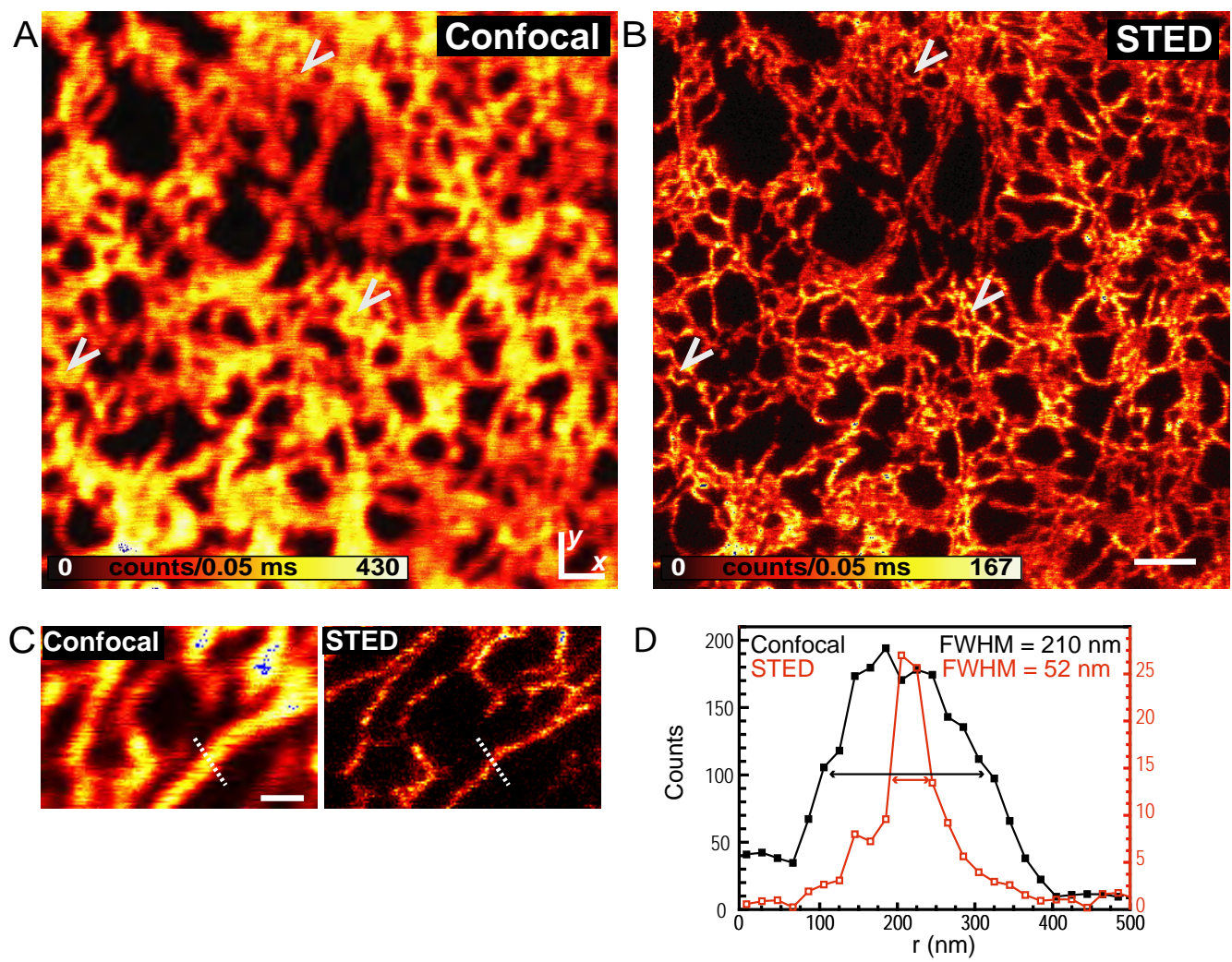

Figure 3.2: Subdiffraction-resolution imaging of the ER in a living mammalian cell. Shown are confocal (A) and simultaneously recorded (B) STED (x, y) images of the ER marked by the fluorescent protein Citrine targeted to the ER (raw data: $16.3 \mathrm{~mW}$ STED focal intensity). Scale bar $1 \mu \mathrm{m}$. The arrows point out rings formed by the tubular network of the ER, which are visible only in the STED image. (C) Confocal and corresponding STED image revealing features of $52 \mathrm{~nm}$ FWHM as the line profiles (D) show (raw data: $35.7 \mathrm{~mW}$ STED focal intensity), indicating that the lateral resolution in the STED image is below $50 \mathrm{~nm}$. Scale bar $500 \mathrm{~nm}$. 
Figure 3.2 shows the protein distribution of Citrine targeted to the ER in a living PtK2 cell. By switching the STED beam on and off, line by line, both the confocal (A) and the STED image (B) were recorded quasi-simultaneously. Whereas in the confocal reference, mainly larger areas can be seen, the STED image distinguishes single tubules or ER elements (compare arrowheads). With an average STED Power of $16.3 \mathrm{~mW}$ spread across the area of the doughnut, the smallest details to be seen are around $50 \mathrm{~nm}$ full-width-half-maximum (FWHM) (see figure 3.2D). Because the image is a convolution of the actual structure with the PSF used for imaging, this represents an upper boundary of the resolving power of the system (i.e., $\Delta r<50 \mathrm{~nm}$ ). For comparison, confocal imaging results in structures with an FWHM of around $200 \mathrm{~nm}$.

To further analyze the resolving capacity of the microscope, the fact that the obtainable resolution, meaning the FWHM of the effectively exciting spot, can be arbitrarily tuned in STED microscopy simply by adjusting the intensity of the depletion beam was exploited (see equation 1.2). For different STED intensities, nanoscopic images of the ER were taken and the FWHM of single tubules were determined. Additionally, the effective PSF was calculated using equation 1.2, $I_{\text {sat }}$ was determined by measuring the fluorescence inhibition on single ER tubules with increasing intensity of a regularly focused STED beam. At a timeaveraged power $P_{\text {sat }}=0.9 \mathrm{~mW}$, the fluorescence dropped by half. Figure 3.3 shows both the FWHM of the effective excitation PSF and the FWHM of the tubules analyzed in the images. For lower STED intensities, a good agreement between the calculated PSF and the measured FWHM can be seen. However, for large values of $I$, the measured FWHM does not decrease any more (in contrast to the calculated FWHM); meaning that the STED image largely reproduces the actual ER network.

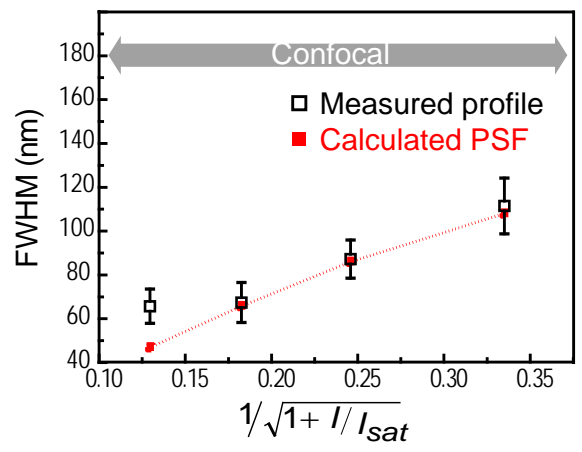

Figure 3.3: FWHM of the calculated effective STED point-spread function (PSF) and of the measured profile of an ER tubule versus the inverse of the square root of the STED beam intensity $I$. The measured FWHM values are based on 125 line profiles measured per individual value $I$ in 5 different images; the error bars represent the quadratic mean of the respective standard deviations. For lower STED intensities $I$, the measured size of the ER elements decreases with the sharpening of the PSF, as expected from the square-root law, whereas for high STED intensities, corresponding to a calculated FWHM of around $50 \mathrm{~nm}$, no further decrease in the measured profile is observed, implying that the ER elements are, on average, $50 \mathrm{~nm}$ in width. 


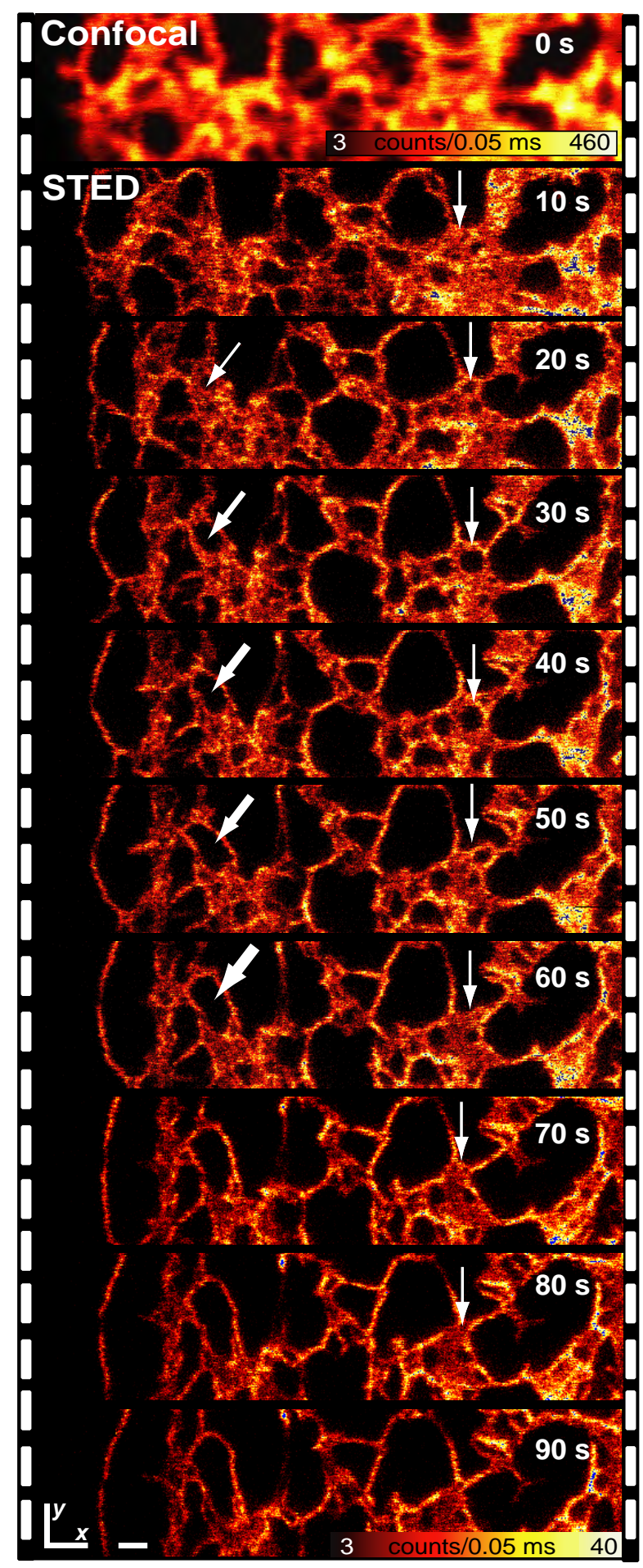

Figure 3.4: Dynamics of the ER imaged with subdiffraction resolution by STED. The images $(10 \mathrm{~s}$ recording time each) were recorded consecutively, showing the movement of the ER with a resolution of $50 \mathrm{~nm}$. The first image (time point $0 \mathrm{~s}$ ) is a confocal reference image. The arrows indicate striking structural changes during the recording of the movie. (Scale bar $500 \mathrm{~nm}$ ) 
In many biological experiments, not only the momentary structure, but the change in structure due to some kind of alteration in the environment is of interest. When working with fixed samples, only snapshots can be taken by imaging cells which were fixed at the appropriate timepoints. In contrast, live cell nanoscopy opens up the possibility of also imaging structural changes in real time with subdiffractive resolution. However, this enhances the requirement on the photostability of the fluorophore: Instead of taking only a single image as is usually done in fixed, immunostained samples, several images of the same area are now needed to get information about the structural changes.

One example of temporal dynamics is presented in figure 3.4, where the endoplasmic reticulum is imaged in several frames over a timespan of $90 \mathrm{~s}$. This time series vividly reveals the structural changes of the ER network, such as the formation and vanishing of enclosures.

Since the fluorescent protein is fused to an ER-targeting and an ER-retention signal, which gives rise to free diffusion within the whole ER network, no drop of brightness is observed over the STED images; potentially photobleached fluorophores in the imaged area are subject to a fast exchange.

The setup can also be used to nanoscopically observe the influence of (chemical) stimulation on living cells. Figure 3.5 shows the dynamics of the ER upon addition of Filipin III, a polyene macrolide which is known to break down the ER [53]. The exact mechanism of this is still unknown; probably, the cholesterol sequestering effect of Filipin III plays an important role. Filipin III was added to the mounted sample at a concentration of $10 \mu \mathrm{g} / \mathrm{ml}$ at $\mathrm{t}=0 \mathrm{~s}$, before high-resolution images were taken at intervals of $80 \mathrm{~s}$. In this time series the gradual breakdown of the ER network and the concomitant formation of large, fast-moving enclosures can be followed with a resolution of $50 \mathrm{~nm}$.
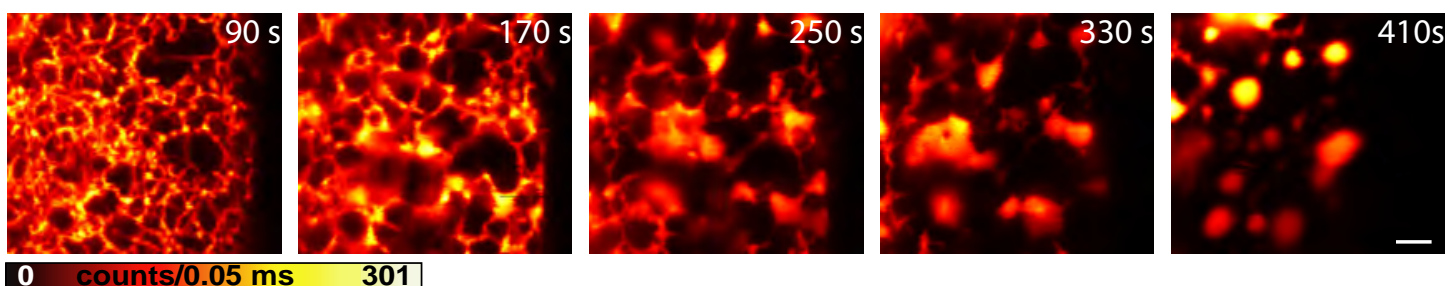

Figure 3.5: The effect of Filipin III on the ER of living mammalian cells, monitored with superresolution. Filipin III was added to PtK2 cells expressing Citrine targeted to the ER at the timepoint zero; afterwards, diffraction-unlimited STED images were taken every $80 \mathrm{~s}$, until the breakdown of the ER to large, fast moving vesicles is completed. Scale bar $1 \mu \mathrm{m}$.

These examples give a first glance as to what is possible by using fluorescent proteins as fluorescent markers for live cell STED imaging. The actual size of the endoplasmic reticulum, which so far has only been determined by using electron microscopy, was measured within the living cell. Therefore, fixation, which can often be the cause for artifacts, becomes 
obsolete. Furthermore, movements and structural changes over time or induced by some external stimulus can be followed with subdiffraction resolution.

\section{The Cytoskeleton}

As discussed above, the free diffusion of the fluorescent protein evades problems regarding photobleaching. However, this is not a prerequisite for live cell STED imaging using fluorescent proteins.

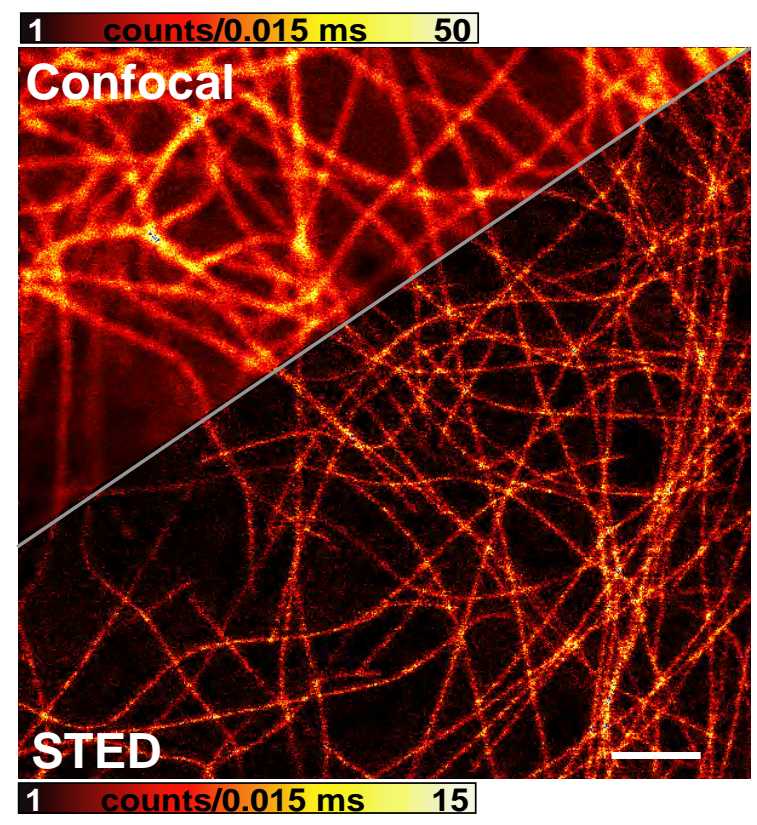

Figure 3.6: Subdiffraction resolution fluorescence imaging of Citrine-labeled microtubules inside a living PtK2 cell. Scale bar $2 \mu \mathrm{m}$.

Figure 3.6 shows a rather dark example, namely the tubulin-structure of PtK2 cells labeled with Citrine by fusing it to $\alpha$-tubulin. In contrast to immunostaining of microtubules, these structures tend to be fairly dim, because the fusion of the comparatively large fluorescent protein to tubulin hinders the effective polymerization of the microtubules. Therefore, only cells with a low degree of overexpression can still form the well-known tubulinstructure. Nevertheless, as can be seen in the lower right part of figure 3.6, applying STED reveals the tubular structures much more clearly than the confocal image does. Single fibers which are close to each other can be discerned only in the STED image.

This shows that STED microscopy of living specimen is not limited to organelles like the $\mathrm{ER}$, but can also be applied to less bright structures like the cytoskeleton where the protein is not freely diffusing. Therefore, a great number of structures are eligible for live cell STED imaging. 


\section{Other Yellow Fluorescent Proteins as a STED Label}

All the examples shown so far utilized the yellow fluorescent protein Citrine as a label. However, in general, any fluorescent protein tested until now can be used; just the signal and the obtainable resolution with a sufficient signal-to-noise-ratio vary.

\section{EYFP}

EYFP, the probably most widely used yellow fluorescent protein, is also a valuable fluorophore for STED microscopy. Since the excitation- and emission properties are very similar to Citrine, the same microscope settings can be used.
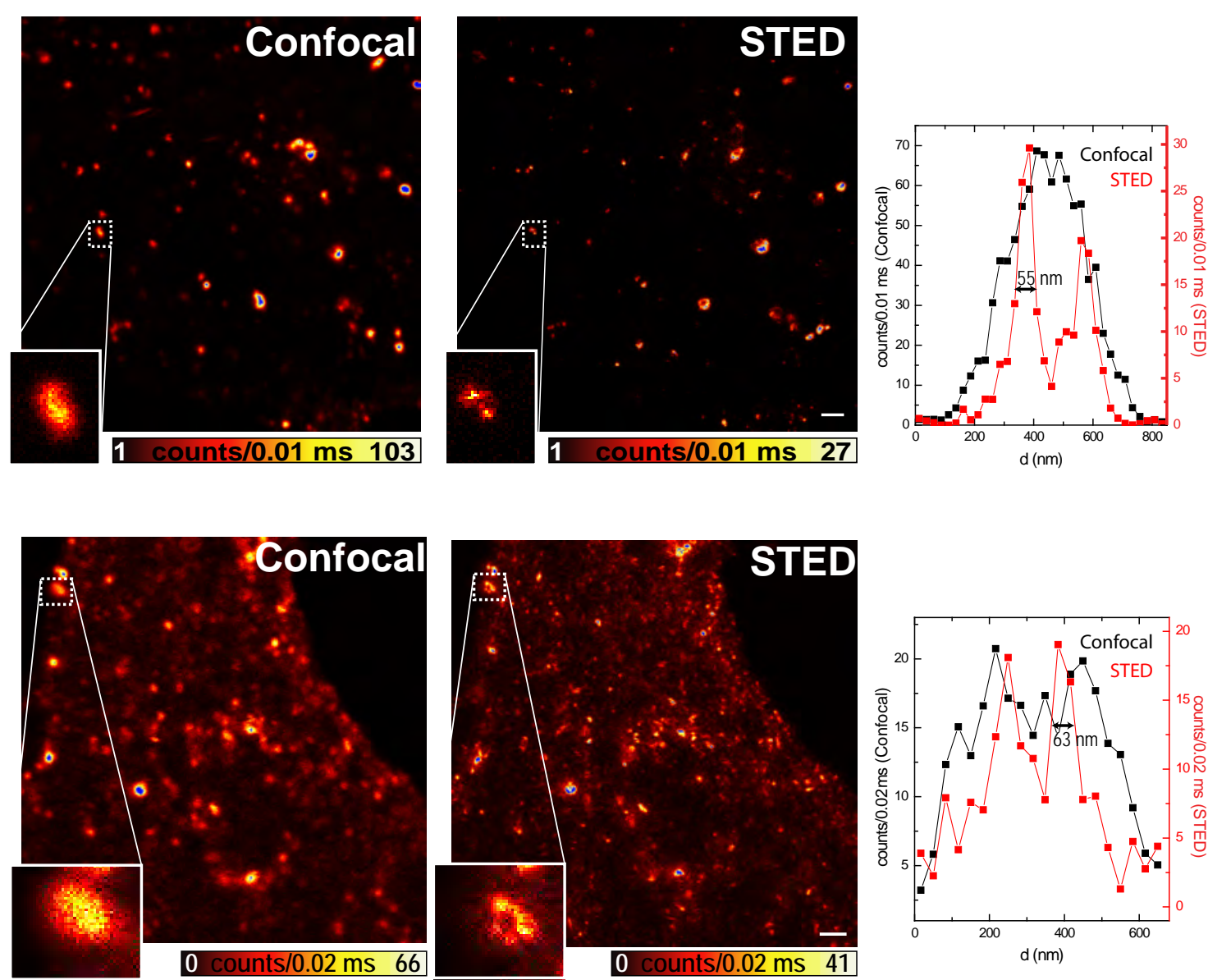

Figure 3.7: Comparison of the performance of the yellow fluorescent proteins Citrine and EYFP: Living mammalian cells expressing K-cadherin fused to Citrine (upper part) and EYFP (lower part). In both STED images, a substantial increase in resolution is obtained, as clearly demonstrated in the enlarged details. The line profiles (right) which were taken through the clusters in the magnifications show that small features of $55 \mathrm{~nm}$ (upper image) and $63 \mathrm{~nm}$ (lower image) can be resolved. Scale bars $1 \mu \mathrm{m}$. 
Figure 3.7 shows an example where K-cadherin was labeled with Citrine (upper part) and EYFP (lower part). Cadherins are a class of type-1 transmembrane proteins which mediate cell adhesion [54]. They derive their name from their calcium dependence (calciumdependent adhesion molecules). The superfamily of cadherins consists of several proteins, which are expressed in different tissues. They all share the extracellular calcium-binding domain, which is formed by the so-called cadherin repeats. Probably the best studied variant, E-cadherin, which is expressed in epithelial cells, is believed to play an important roll in cancer development. E-cadherin downregulation decreases the strength of cellular adhesion within a tissue, resulting in an increase in cellular motility, which may lead to cancer metastasis [54]. Here, the distribution of K-cadherin, which is normally expressed in kidney tissue, in living PtK2 cells is shown.

For both Citrine- and EYFP-labeled samples, the STED image reveals small details much more clearly. The line profiles (figure 3.7, right) show structures with a diameter of $55 \mathrm{~nm}$ in the Citrine-labeled sample and $63 \mathrm{~nm}$ in the EYFP-labeled sample. However, a direct comparison of the performance of the proteins cannot be done based on these data, because the size of the imaged structures is not only dependent on the resolution of the setup, but also on the size of the structure itself. In the example presented here, the size distributions of the clusters is very broad, as can be seen in the fluorescence images: Clusters with different sizes are observed. Therefore, $55 \mathrm{~nm}$ and $63 \mathrm{~nm}$ are only upper boundaries for the resolving capacity of the respective system.

Based on these findings, EYFP has also been used to examine organotypical brain slices of transgenic mice expressing EYFP-labeled hippocampal neurons by the means of STED microscopy [55]. So far, a lot of the ongoing studies in the neurosciences are performed using confocal microscopy supplemented by electron microscopy to resolve fine structures not accessible by conventional light microscopy. Using STED microscopy thereby not only simplifies the experimental approach, it also provides the opportunity to monitor structures beyond the diffraction limit in living tissue. Since morphological changes on the nanoscale are thought to play an important role in the function and plasticity of synaptic connections, this approach opens up new possibilities in many neurobiological experiments [55]. 


\section{Venus}

Also Venus, a yellow fluorescent protein with increased maturation speed, can be used for STED imaging: Compared to EYFP, Venus contains the mutation F64L, which at $37{ }^{\circ} \mathrm{C}$ greatly accelerates oxidation of the chromophore, the rate-limiting step of maturation [56]. This makes Venus a valuable tool for biological experiments where timing is crucial. The STED microscope settings for imaging Venus-labeled samples are the same as for imaging Citrine- or EYFP-labeled samples, because their spectra are very similar.

Figure 3.8 shows an example where PC12 cells expressing a fusion protein of neuropeptide Y (NPY) and Venus were imaged. NPY is a 36 amino acid peptide neurotransmitter, which is involved in various physiological processes in the brain, including the regulation of energy balance, memory and learning [57]. A whole cell was imaged in 14 frames, taking one frame every 10 seconds. The first frame is a confocal reference. As can be seen especially in the enlarged detail, the labeled vesicles containing NPY are not stationary but move through the cell.

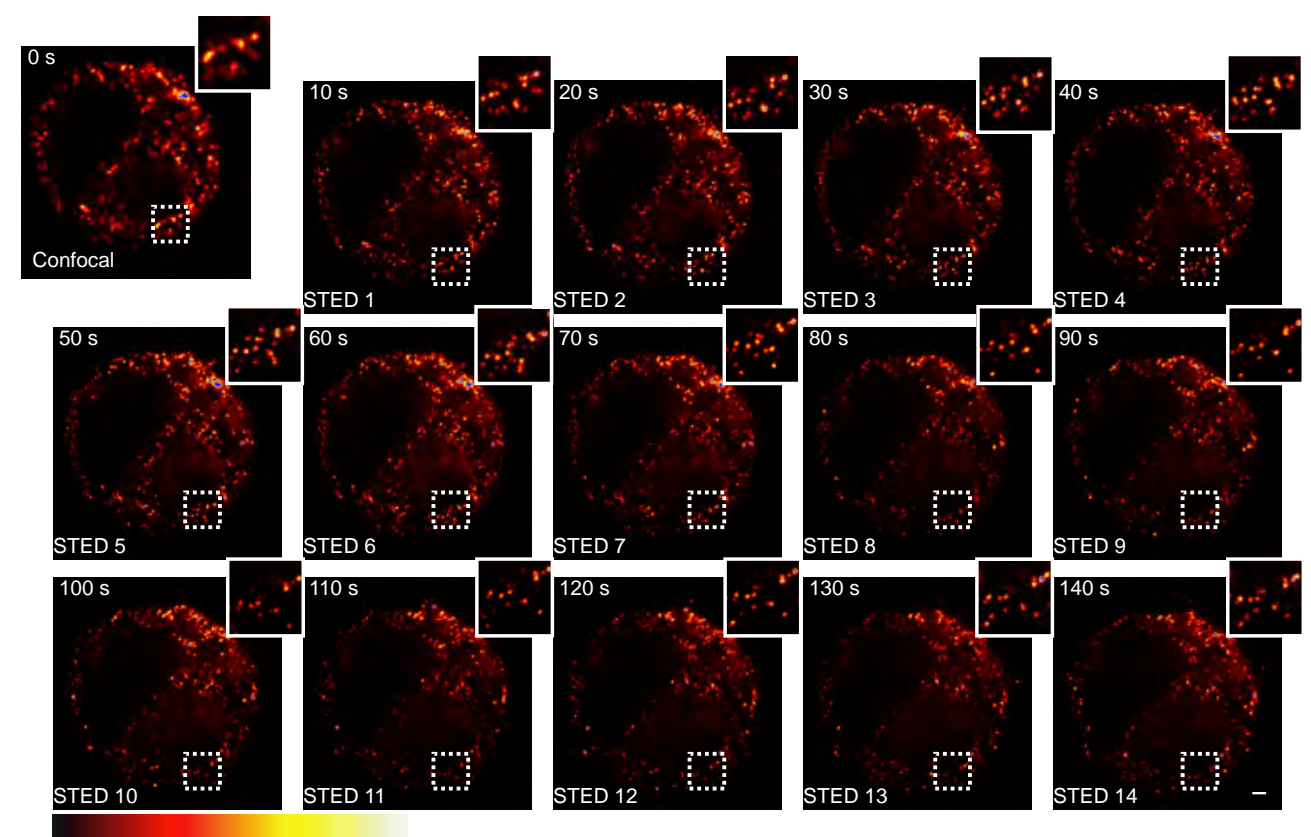

Figure 3.8: Series of STED images of a PC12-cell expressing NPY labeled with Venus, a fast maturing yellow fluorescent protein. The video reveals the movement of NPY-vesicles through the cell with subdiffraction resolution. Scale bar $1 \mu \mathrm{m}$.

This shows that fluorescent proteins other than Citrine and EYFP can also be successfully employed as fluorescent markers for live cell STED microscopy. Therefore, if the biological experiment requires, for example, a fast-maturing fluorescent protein, this does not preclude superresolution imaging. 


\subsubsection{Axial Resolution Enhancement}

In the images presented so far, only the doughnut-shaped STED focus for resolution enhancement in the lateral direction was applied. However, by implementing the so-called z-doughnut (see figure 2.2), where the two intensity maxima are located along the optical axis featuring a zero in the very center, resolution enhancement in the z-direction within living samples can also be achieved. This is necessary for addressing structures which are strongly folded in three dimensions. An example is shown in figure 3.9, where a PtK2 cell expressing the yellow fluorescent protein Citrine targeted to the ER is presented. In contrast to the images of the ER shown so far, not the flat outer regions of the cell were imaged, but an area close to the nucleus, where the cell shows a substantial extension along the z-axis as well. In the STED image recorded with the z-doughnut, tubules stacked above each other are resolved. In contrast, the corresponding confocal image cannot distinguish stacked elements from interconnected tubules. Taking line profiles across single tubules show a resolution of $150 \mathrm{~nm}$ of the STED microscope along the optical axis, which represents a 3-fold improvement over the optical sectioning capacity of a confocal microscope, which is around $450 \mathrm{~nm}$ for visible light.

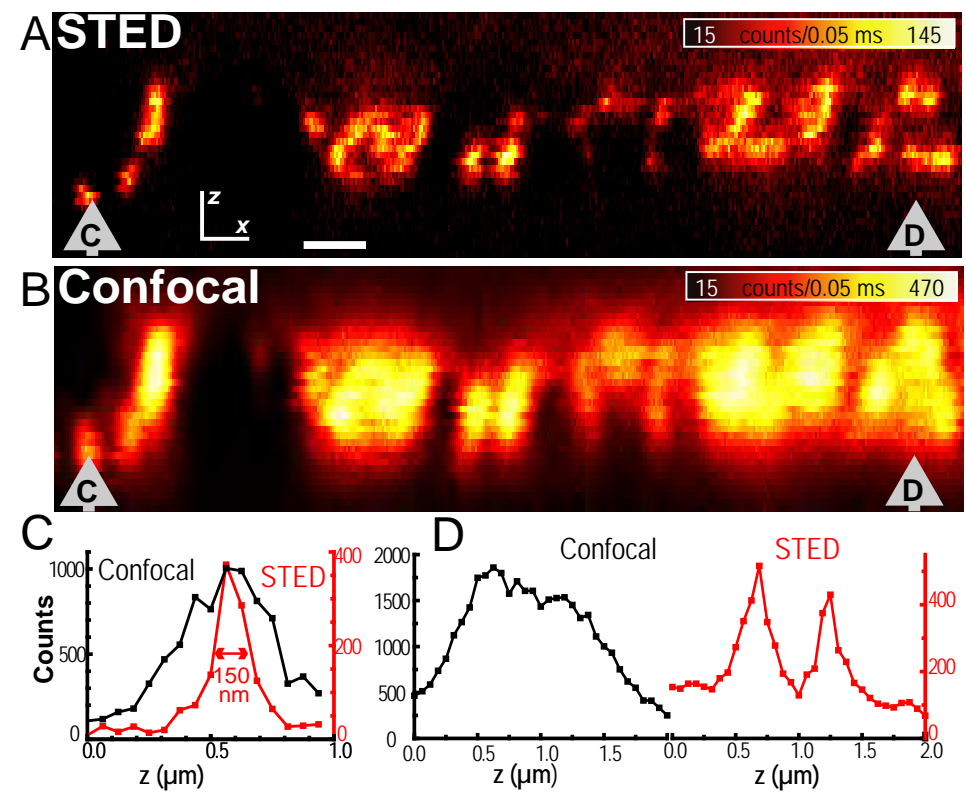

Figure 3.9: Axial superresolution and improvement of optical sectioning inside a living cell using STED. An axial STED image (xz) of densely packed ER tubules (A) imaged by STED microscopy reveals single, stacked tubules, whereas in the confocal image (B), the same substructure is not revealed. Scale bar $500 \mathrm{~nm}$. (C) Line profile along the $\mathrm{z}$ direction (sum of four lines) of a single ER tubule at the marked position of the STED (A) and the confocal (B) images, respectively, revealing an axial FWHM of $450 \mathrm{~nm}$ in the confocal reference and $150 \mathrm{~nm}$ in the STED image. (D) Intensity profile along the $\mathrm{z}$ axis (sum of four $\mathrm{z}$ profiles) demonstrates the ability of the STED microscope to discern features that cannot be separated in the confocal reference. 
Since the effective focal spot functions as a noninvasive 3D probe, the improvement in $\mathrm{z}$ resolution also yields an improvement in optical sectioning. This is a major advantage of STED microscopy over other nanoscopy methods based on stochastic switching of single molecules: Unless the switching is effected by a multiphoton process, it does not provide optical sectioning, so that - unlike in STED - an arbitrary plane in the sample can not be addressed.

\subsubsection{Imaging Living Yeast}

Besides the possibility of performing live cell experiments, the usage of fluorescent proteins offers other advantages compared to immunocytochemistry. For example, immunostaining in yeast does not work as well as in mammalian cells, because yeast cells posses a thick cell wall, which requires a very stringent treatment to become permeable for antibodies. Therefore, fluorescence microscopy studies of yeast are often done using fluorescent proteins. As discussed in the previous paragraphs, yellow fluorescent proteins are well suited for STED microscopy. They were used in this work to get the first nanoscopy images of functional structures within a living yeast cell.

Figure 3.10 shows fluorescence images of living yeast cells expressing Citrine fused to different membrane proteins. Cells were mounted in $1 \%$ agarose, in which the yeast survives for a certain amount of time, while keeping the cells in place. The images are optical sections through the spherical yeast cell, showing the outer membrane as a ring-like structure.
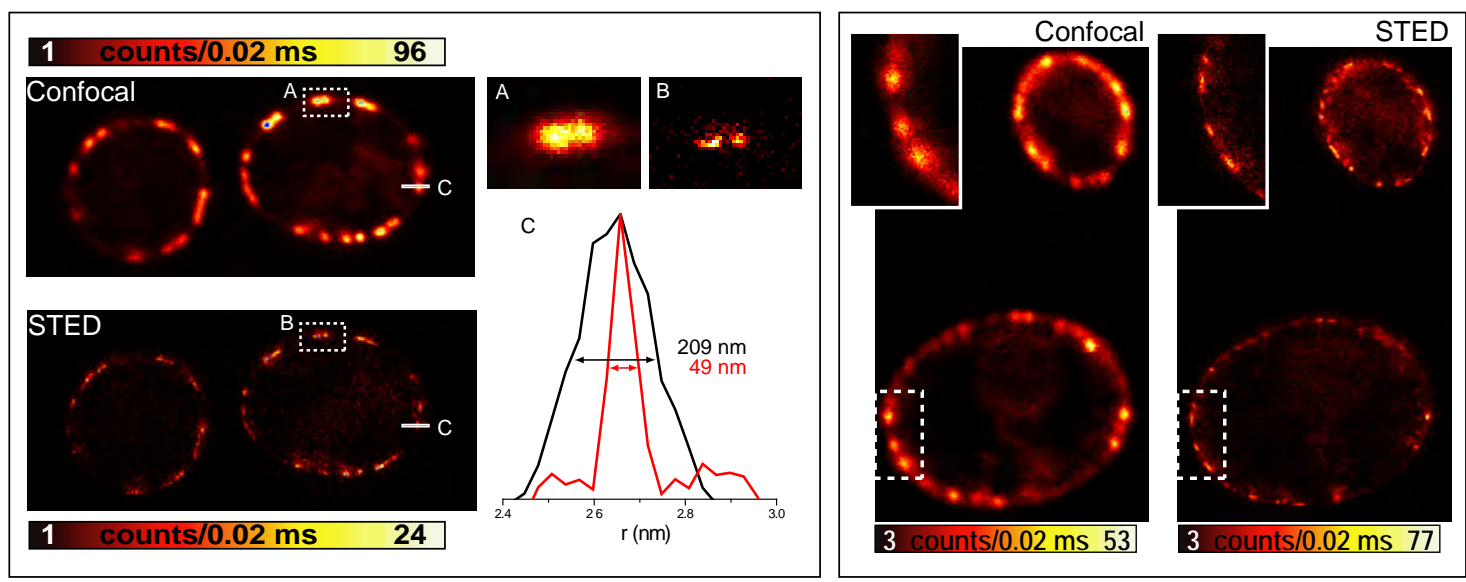

Figure 3.10: Living yeast cells, expressing Citrine-fusion proteins. Left: Pill, one main component of the eisosome, is located in the cell membrane. STED imaging proves that these structures are less than $50 \mathrm{~nm}$ thick, which can not be determined by using conventional light microscopy. Scale bar $1 \mu \mathrm{m}$. Right: Nce102 shows a similar distribution as Pill. Here also, the STED image exposes the structures in more detail than the confocal image does. Scale bar $1 \mu \mathrm{m}$.

On the left hand side, yeast cells expressing Citrine-Pill are shown. Pill is one of the two main eisosome subunits [58]. The term eisosome describes immobile protein fractions 
located at the plasma membrane which mark the sites of endocytosis. They have been identified recently; before, endocytosis was thought to happen randomly at the plasma membrane [59]. In the confocal image (left), the eisosomes appear as structures with a thickness of around $200 \mathrm{~nm}$ located to the cell membrane. However, the STED image (right) reveals that these structures are smaller than that. Analyzing the FWHM of individual line profiles through these clusters results in a thickness of about $50 \mathrm{~nm}$.

The expression of Nce102 fused to Citrine shows a similar distribution (figure 3.10, right): Nce102 is an integral membrane protein with a thus far unknown function [60]. The STED image again reveals structures with a thickness below $50 \mathrm{~nm}$, whereas in the confocal reference, due to diffraction only structures of around $200 \mathrm{~nm}$ are visible.

These are the first examples of superresolution imaging of functional structures within living yeast cells. This shows that it is possible to perform the microscopy studies of yeast so far done by confocal microscopy with a STED microscope also, thereby improving the resolution by a factor of 4 in the lateral direction.

\section{Summary}

The data presented in this part are the first examples of live cell STED imaging using fluorescent proteins as a label. Using the yellow fluorescent protein Citrine, a lateral resolution of about $50 \mathrm{~nm}$ was achieved while imaging different structures within living cells. Structural changes over time or induced by an external stimulus were also monitored with subdiffraction resolution. By applying a different phasemask to shape the STED focus, resolution enhancement in the axial direction was obtained as well. EYFP, the probably most prominent yellow fluorescent protein, and Venus, a fluorescent protein not optimized for photostability but for short maturation times, can also be used for STED imaging. In addition to examining different mammalian cell lines, the first STED images of functional structures in living yeast were presented.

Summing up, these examples reveal that a lot of the ongoing studies within living cells using confocal microscopy can also be performed using a STED microscope, resulting in an increased spatial resolution and thereby revealing more information. 


\subsection{Live Cell STED Imaging using Organic Dyes}

As was shown in the last section, it is possible to perform STED imaging of living cells using autofluorescent proteins with both lateral and axial resolution enhancement by factors of 4 and 3 , respectively, as well as to nanoscopically monitor structural changes in living cells over time.

However, the possibilities for changing and improving the chromophore in fluorescent proteins is limited. A lot of effort has been invested in mutating these proteins and screening for improved variants, which gave rise to the above mentioned mutants EYFP, Venus and Citrine. Nevertheless, because the chromophore is built out of several amino acids contained in the protein, there are severe constraints regarding the chromophore structure. In contrast, organic dyes can be based on different structural backbones; furthermore, a huge amount of modifications can easily be introduced by chemical synthesis. These possibilities led to the development of a large number of organic dyes with very diverse properties; in particular, dyes optimized for the specific requirements of STED microscopy have been developed. Therefore, it would be advantageous to also use these dyes for live cell imaging. The key issue in this regard is how to specifically label the structure of interest with the fluorophore within the living cell.

As already presented in chapter 1.4 .3 , proteins with a high affinity for a specific substrate have recently been developed, which can - like fluorescent proteins - be fused to other proteins. Incubation with their substrate carrying the desired fluorophore leads to labeling of the structure of interest. The choice of the best suited tagging protein depends on the application; important features to be considered include the size of the tag, membrane-permeability of the substrate, stability of the linkage to the dye and restrictions on the dyes which can be used. Here, two approaches are used, allowing for the free choice of the fluorophore, which is covalently bound to the tagging protein. In the first case, an enzyme is used to transfer the fluorophore to the tagging protein (chapter 3.2.1), whereas in the second example, a selflabeling reaction of the tagging protein with the modified fluorophore is employed (chapter 3.2.2. 


\subsubsection{Enzyme-Mediated Labeling using Acyl Carrier Protein}

As described in chapter 1.4.3, fluorophores attached to coenzyme A can be enzymatically transferred to Acyl Carrier Protein (ACP) [43]. ACP contains phosphopantetheine as a covalently bound prosthetic group, which is the site of attachment for various intermediates within metabolic reactions. The transfer of phosphopantetheine from coenzyme A to Ser36 of ACP is mediated by the enzyme phosphopantetheine transferase (pptase), which also tolerates modifications at the thiol-group of coenzyme A [43]. Therefore, by attaching a fluorophore to coenzyme A at that position, the fluorophore gets transferred to ACP. By expressing a fusion protein of the protein of interest and ACP and incubation with both the fluorophore-containing coenzyme A and pptase, the protein of interest becomes fluorescently labeled.

Different fluorescent organic dyes have been coupled to coenzyme A in this work; a complete compilation can be found in chapter 2.2.3. Several of these dyes have already been successfully used for STED microscopy, but the labeling via tagging proteins now also allows for live cell imaging using organic dyes. Importantly, fluorescent dyes in the far-red regime can be used as well. To couple the dye to coenzyme A, the maleimide-derivative of the dye is added to a solution of coenzyme A, which reacts in a one-step reaction to the substrate for ACP. Figure 3.11 shows the product CoA-Atto565, which was predominantly used in this work. Excitation is performed using a pulsed 532nm laser diode, while the optimal STED wavelength is $660 \mathrm{~nm}$.
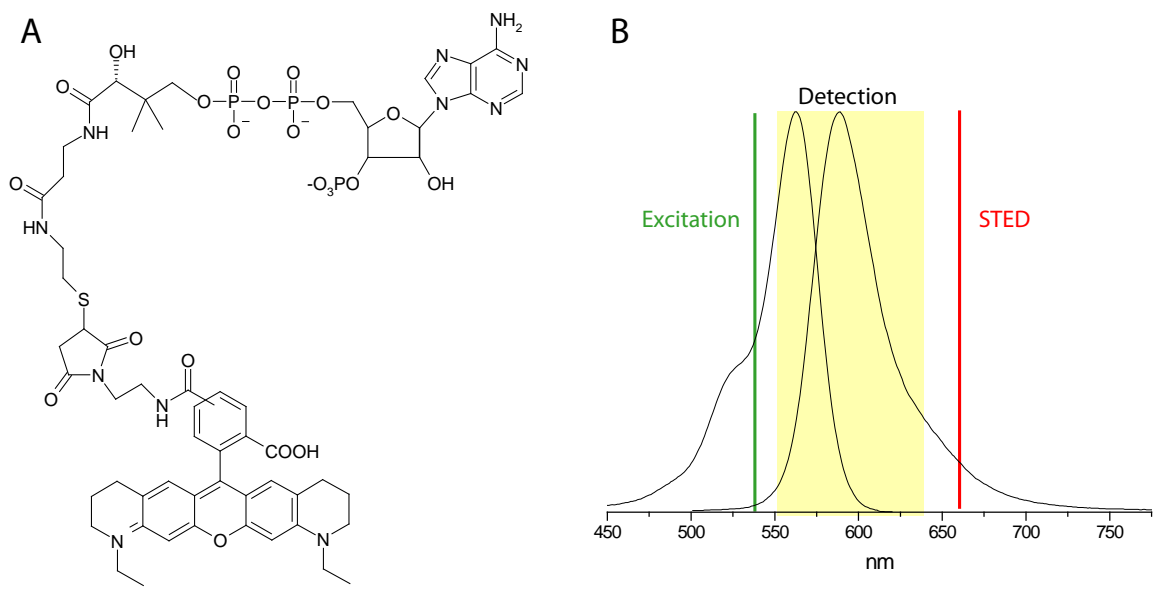

Figure 3.11: Structure of coenzyme A carrying Atto565, which can be used for the covalent labeling of the surface of living cells using the ACP-tag (A). In the spectrum (B) the excitation and STED wavelength, as well as the detection range used for STED imaging are indicated.

As mentioned before, this approach is limited to the cell membrane. However, being the place for many biologically relevant processes like cell adhesion, signaling or cell-cellcontact, the cell membrane attracts a lot of attention. Figure 3.12 presents an example where 
ACP is attached to the cell membrane of a Hela cell by a GPI-anchor. In this case, Atto647N was used for labeling; excitation was performed at $635 \mathrm{~nm}$, while STED light at $760 \mathrm{~nm}$ was provided by a Ti:Sapphire laser. The filopodia, the extensions at the outer rim of the cell, can be resolved in more detail by using STED, as the comparison of the two images shows.
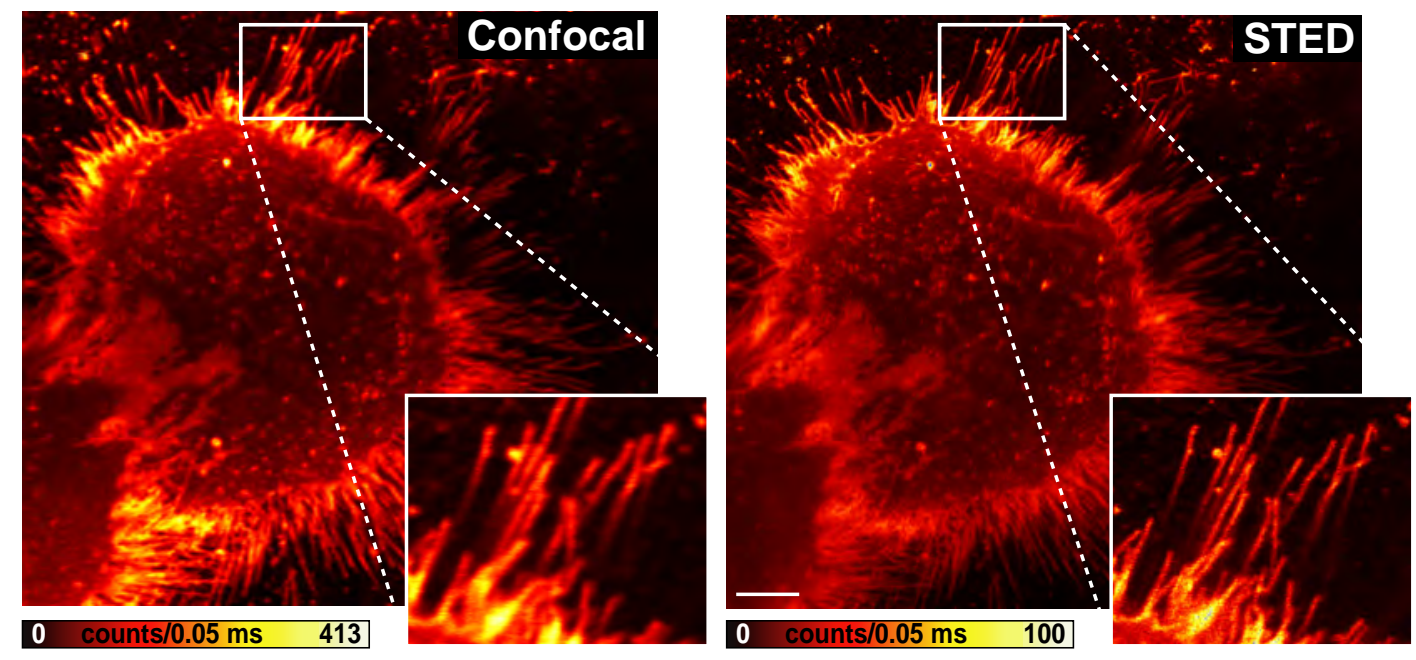

Figure 3.12: Living HeLa cells, expressing GPI-anchored ACP labeled with Atto647N. The STED image (right) reveals individual filopodia, which can not be distinguished in the confocal image (left). Scale bar $5 \mu \mathrm{m}$.

A big advantage of using ACP is the small size of the tagging protein: consisting of only 77 amino acids, interference with the fusion protein is less likely than when using autofluorescent proteins built of 238 amino acids.

It has thus been demonstrated that fluorescence labeling with ACP using different organic dyes allows for STED imaging of living cells. Advantages compared to using fluorescent proteins, as discussed above, are, in addition to the smaller size, the free choice of the fluorophore. 


\subsubsection{Selflabeling Tags for Intracellular Applications}

To study also intracellular structures, a different tagging approach is versatile: human alkylguanine DNA alkyl transferase (hAGT) selectively binds modified alkylguanines and related structures [44]. By incubating cells expressing hAGT fusion proteins with benzylguanines carrying a fluorophore as a substrate, a covalent attachment of the fluorophore to the tagging protein takes place.

Derived from a DNA repair protein, which removes alkylgroups from alkylated $\mathrm{O}^{6}$ guanine nucleotides by transferring them to a reactive cysteine, hAGT has been engineered to become smaller $(\approx 20 \mathrm{kD})$, to react faster with its benzyl guanine substrates and to remove its affinity for DNA. Here as well, in principle, any organic dye can be used as a substituent, leading to a broad variety of substrates for hAGT. Nonetheless, for labeling of intracellular structures, membrane permeable substrates have to be used, which narrows down the substrate availability.

Important features affecting the membrane permeability include the size, hydrophilicity and charge of the dye. Generally speaking, small and not highly charged dyes are more likely to cross the membrane. However, a reliable prediction about its behavior has so far not been possible. In addition to the dye, the attached moiety which is recognized by hAGT influences also the permeability; but again, predictions of which moiety to use with which dye are currently very unreliable. In this work, two different substances which can be recognized by hAGT were used: $\mathrm{O}^{6}$-benzylguanine (BG) and 4-(4-aminomethyl)benzyloxy)6-chloropyrimidine-2-amine (CP). Figure 3.13 shows the reaction of the respective amines with an N-hydroxysuccinimidyl-modified dye, yielding substrates for hAGT:

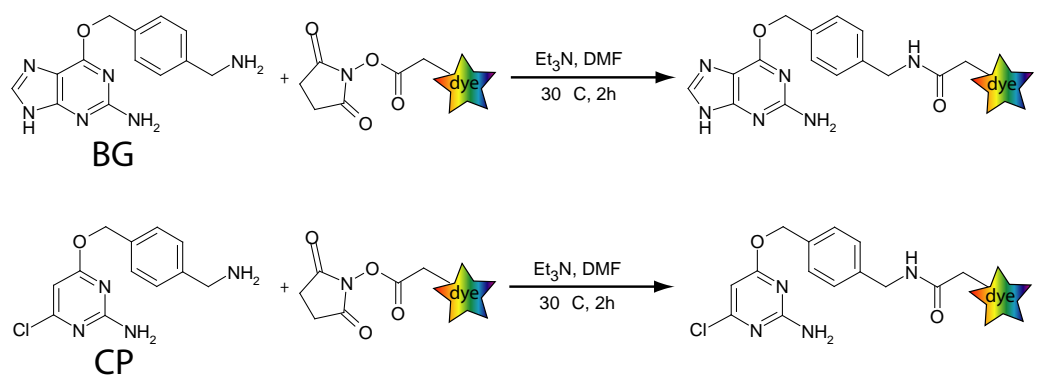

Figure 3.13: Reaction scheme for synthesis of benzylguanine- (BG, upper part) and chloropyrimidin- (CP, lower part) modified fluorophores, which can be used for covalent labeling of hAGT-fusion proteins.

This allows for the fast and easy synthesis of substrates with different fluorophores. In addition to membrane permeability, unspecific background is an important criterion for evaluation of the dyes. For BG-coupled substrates, fluorescence quenching can be observed for several dyes by photoinduced electron-transfer from the dye to the guanine [61]. Upon binding to hAGT, the guanine is detached, leading to a disruption of the fluorescence quenching. 
This mechanism helps to reduce background fluorescence from unspecifically bound fluorophores.

A compilation of the synthesized and tested conjugates can be found in chapter 2.2.3. Two different dyes proved to be well suited for intracellular labeling in combination with STED imaging: tetramethylrhodamine (TMR) attached to CP and Oregon Green attached to BG. The structures and spectra are shown in figure 3.14 .

\section{CP-TMR}<smiles>CN(C)c1ccc(CNC(=O)c2ccc(O)c(C(=O)NCc3ccc(N(C)C)cc3)c2-c2ccc(N(C)C)cc2OCc2cc(N)nc(Cl)c2)cc1</smiles>

BG-Oregon Green<smiles></smiles>
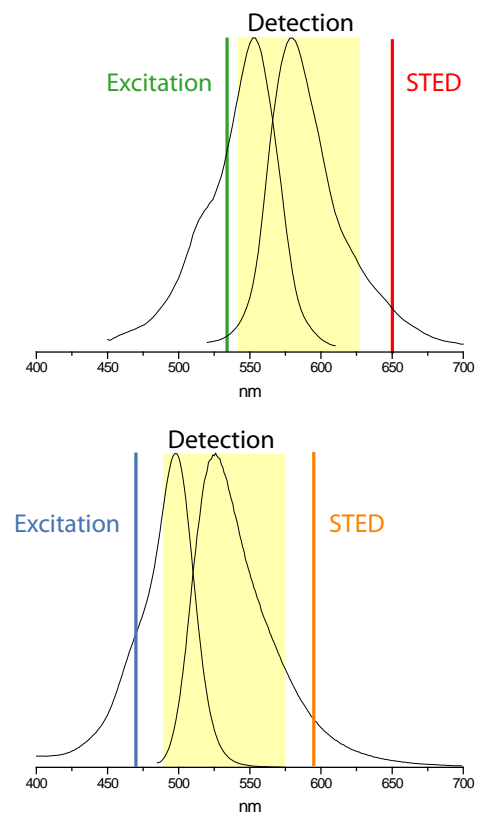

Figure 3.14: Structure and spectra of CP-TMR (top) and BG-Oregon Green (bottom), which were used in this work for the covalent, intracellular labeling of different structures in living mammalian cells. The respective excitation and STED wavelengths as well as the detection ranges are indicated.

In figure 3.15, a PtK2 cell transfected with hAGT fused to connexin-43 and labeled with CP-TMR is displayed. The connexin-family consists of different transmembrane proteins that assemble to form gap junctions [62]. Gap junctions are essential for many physiological processes, such as the coordinated depolarization of cardiac muscle, and proper embryonic development. For this reason, mutations in connexin-encoding genes can lead to functional and developmental abnormalities. As can be seen in both the STED image (figure 3.15A) and the confocal reference (figure $3.15 \mathrm{~B}$ ), which was taken after the STED image by blocking the STED beam only, the protein forms clusters on the cell membrane. Some neighboring clusters can only be resolved in the STED image, as can be seen in the line profile shown in figure $3.15 \mathrm{C}$, because their distance is well below the diffraction limit. To estimate the resolution obtainable by this means, the full width half maxima (FWHM) of line profiles through individual spots in figure $3.15 \mathrm{~A}$ and $3.15 \mathrm{~B}$ were determined, which were then plotted in a histogram (figure 3.15D). The distributions of the FWHM measured in the confocal and 

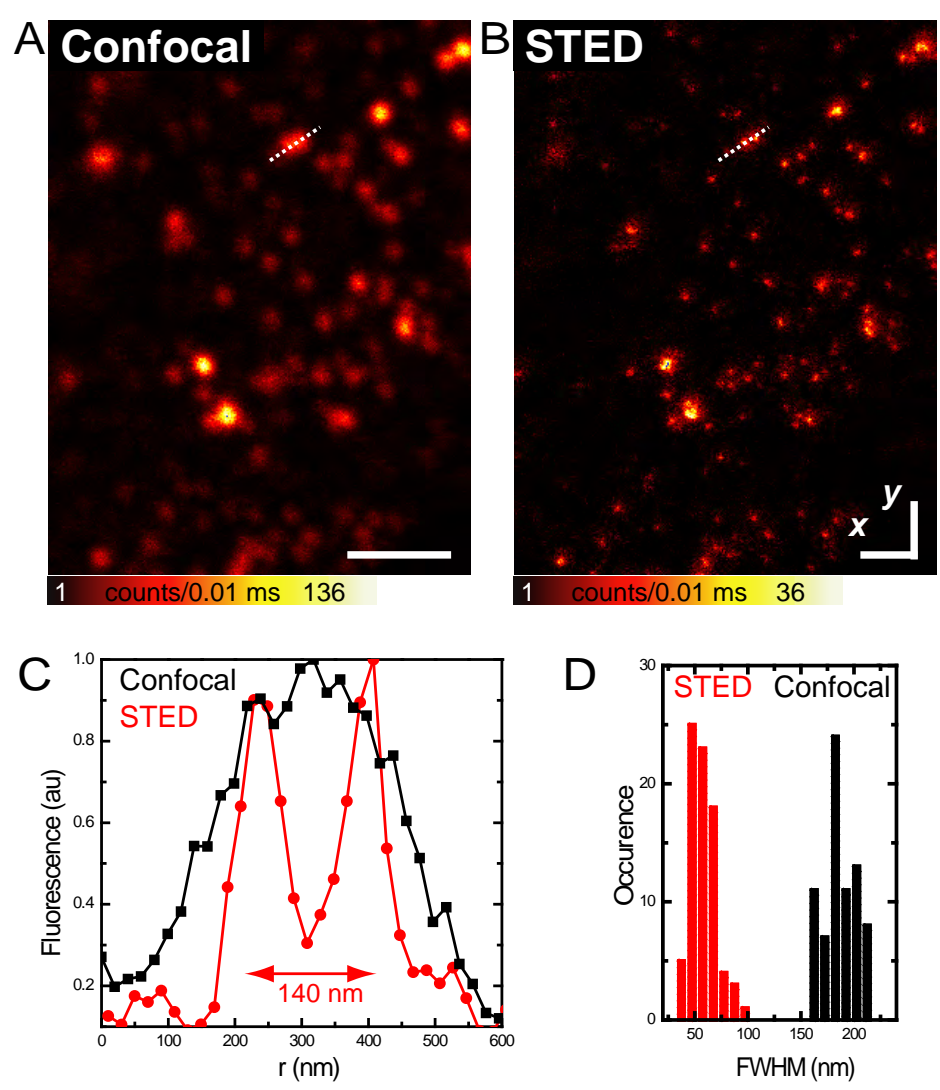

Figure 3.15: Subdiffraction-resolution STED imaging of gap junctions in living PtK2 cells. A and B show a confocal and STED image, respectively, of connexin-43 fused to hAGT and labeled with TMR (raw data, $39 \mathrm{~mW}$ focal intensity of the STED beam). Scale bar $1 \mu \mathrm{m}$. C displays profiles along the marked line in A and B which demonstrate that individual clusters can be discerned by STED which are not separated by confocal microscopy. A histogram (D) of the FWHM of 60 measured line profiles through individual clusters indicates a resolution of at least $40 \mathrm{~nm}$ by these settings. 
STED mode, respectively, are well separated, indicating that the size of the connexin-43 clusters is well below the diffraction limit. The smallest FWHM values measured in the STED image point out that a resolution down to $40 \mathrm{~nm}$ is achieved by this system in living cells. This value is in good agreement with simulations of the effective STED PSF for these dye and wavelength settings.

Again, movies can be obtained by taking several images of the same region. An example is shown in figure 3.16, where movements of connexin- 43 clusters across the cell membrane were monitored with subdiffraction resolution.

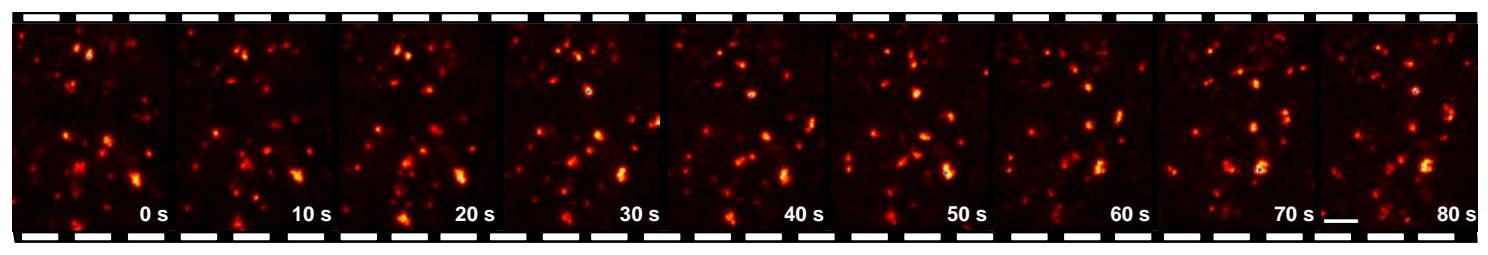

Figure 3.16: Nanoscopic video of the movement of connexin-43 clusters across the cell membrane of a living PtK2 cell. Labeling of the hAGT fusion protein was performed using CP-TMR. Scale bar $1 \mu \mathrm{m}$.

Similar images can be obtained by using Oregon Green. Figure 3.17 shows a PtK2 cell expressing MAP2-hAGT, which was incubated with BG-Oregon Green. MAP2 (microtubuliassociated protein 2) is a protein which binds to the tubulin subunits to regulate the stability of the microtubules [63]. A large variety of MAPs have been identified in many different cell types, and they have been found to carry out a wide range of functions. These include both stabilizing, crosslinking and destabilizing of microtubules, guiding them towards specific cellular locations and mediating the interactions with other proteins in the cell [64]. These proteins have a conserved $\mathrm{C}$-terminal microtubule-binding domain and variable $\mathrm{N}$-terminal domains projecting outwards, which probably interact with other proteins. The interaction of the C-terminal domain with microtubules also explains why - unlike for all other structural proteins examined in this work - only fusions to the $\mathrm{N}$-terminus resulted in the correct localization.
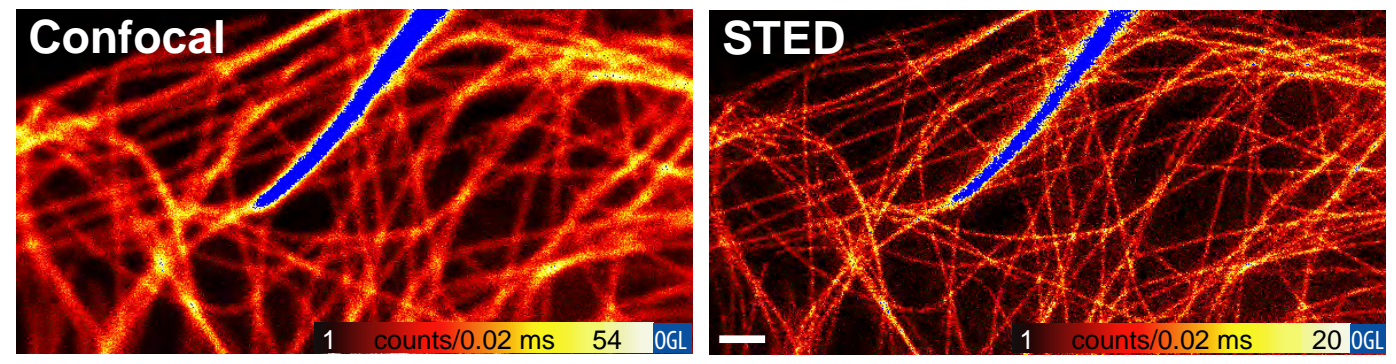

Figure 3.17: Live cell confocal (left) and STED image (right) of microtubule-associated protein (MAP2) labeled with hAGT-Oregon Green. Scale bar $1 \mu \mathrm{m}$. 
Again, in the STED image individual tubules can be revealed, which is not possible in the confocal image. Since Oregon Green has very similar absorption- and emission spectra as yellow fluorescent proteins, the same microscope setup can be used.

As an alternative to hAGT, there is a different protein commercially available: HaloTag (Promega Corporation, Madison, USA) is a dehalogenase which recognizes and binds dyecarrying halogenalkanes [65]. The availability of membrane-permeable fluorophores is similar to hAGT; for example, a membrane-permeable TMR-conjugated substrate also exists. Figure 3.18 shows an example where vimentin, an intermediate filament of the cytoskeleton [34], was labeled with TMR via the Halotag protein in living PtK2 cells. A similar resolution as when using hAGT can be obtained by STED imaging. However, in many cases, the structures were more disrupted than in equivalent samples expressing hAGT (compare figure 3.21). This is probably due to the larger size of the HaloTag protein compared to hAGT.

A
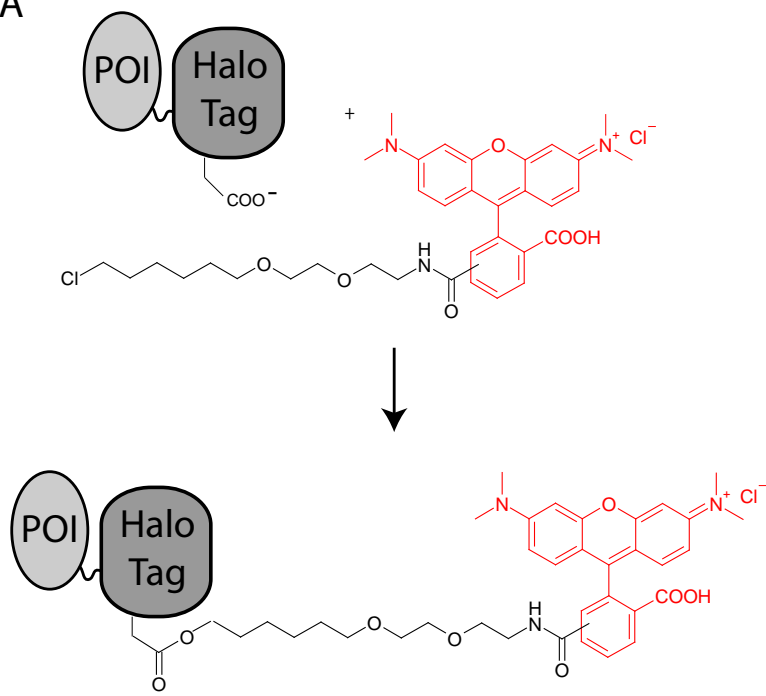

B

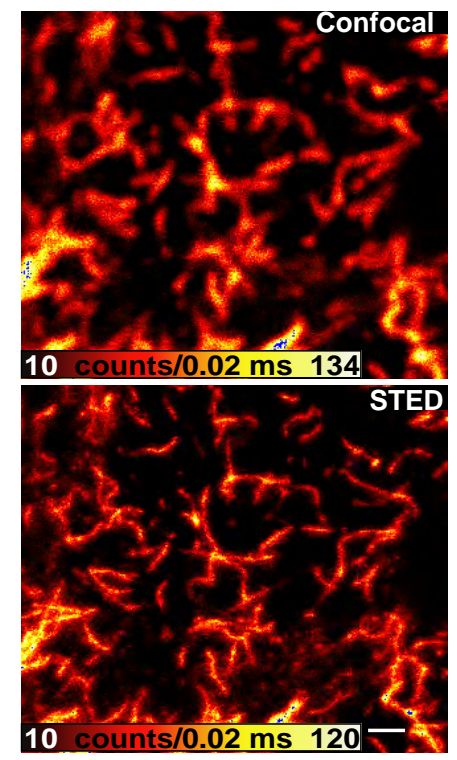

Figure 3.18: Live cell labeling using HaloTag. A: Specific reaction of modified halogen alkanes with HaloTag, which can be fused to any protein of interest. B: Confocal (top) and STED (bottom) image of HaloTag-TMR-labeled vimentin. The STED image reveals the structure much more clearly than the corresponding confocal image. In contrast to vimentin labeled with hAGT or with an autofluorescent protein (compare figure 3.21, the structure is more disrupted, which is probably due to the larger size of the fusion protein. Scale bar $1 \mu \mathrm{m}$. 


\section{Summary}

The examples presented here show that by using different tagging proteins capable of binding modified organic dyes, STED images of living cells can be obtained.

The ACP-tag, which requires addition of the enzyme pptase for binding dye-substituted coenzyme A can be used for imaging structures on the cell membrane. Different substrates were synthesized, carrying various organic fluorophores. The advantage of the ACP-tag is its small size of only 77 amino acids, which is only about a third of the size of an autofluorescent protein.

For imaging intracellular structures, hAGT is a well suited tagging protein. It can in principle also be used in conjunction with any fluorophore. However, here the membranepermeability of the dye has to be considered. STED images within living cells with a resolution down to $40 \mathrm{~nm}$ were achieved using TMR or Oregon Green as a fluorophore. Also, short movies showing the movements of membrane clusters with nanoscale resolution were recorded. 


\subsection{A Simplified Setup for Live Cell Imaging using CW-Lasers}

Probably the most complex and expensive part of the STED microscope is the laser system providing the STED pulses: A Titanium-Sapphire laser (here: MaiTai, Spectra Physics) is needed. For imaging in the visible range, additionally an Optical Parametric Oscillator (OPO) with an enclosed SHG-crystal for frequency-doubling is required, which is pumped by the Ti:Sapphire laser. This configuration is unfortunately not plug-and-play but needs proper alignment.

Recently, other powerful laser sources in the visible range have been developed. One very versatile approach uses whitelight-lasers, which provide easy tuning of the wavelength within the whole visible range [66]. However, for live cell-imaging, this approach is not optimal, because it works with a very low repetition rate (1 MHz compared to $80 \mathrm{MHz}$ of the Ti:Sapphire-OPO System), which increases the acquisition time to the order of several tens of minutes for a $20 \mu \mathrm{m}$ x $20 \mu \mathrm{m}$ image. Instead of going to lower repetition rate, it is therefore more reasonable to go to higher repetition rates or - in the last extreme - to continuous wave (cw). Recently it was shown that STED microscopy can be performed using cw lasers [67]. It works with various organic dyes; however, a strong triplet- or other dark state population may lead to pronounced bleaching via dark state excitation. In addition to the less elaborate laser system, the timing between excitation and STED becomes redundant.

The latest developments in the area of cw-lasers led to the availability of high-power lasers (at least $1 \mathrm{~W}$ ) in the visible range. Therefore, this might be a valuable alternative to the pulsed system for live cell nanoscopy.

As a rule of thumb, the affordable reduction in peak intensity is largely determined by the ratio of the STED pulse duration (here: about $300 \mathrm{ps)}$ ) to the lifetime of the excited state of the fluorophore, which is in the case of the fluorescent protein Citrine about $3.6 \mathrm{~ns}$, i.e. 12-fold.

The fluorophores used for live cell STED imaging in this work required STED wavelengths of $650 \mathrm{~nm}$ (TMR) and $595 \mathrm{~nm}$ (yellow fluorescent proteins, Oregon Green). For the former dye, the widely used Krypton-laser offers a line at $647 \mathrm{~nm}$ with high intensities. STED at $592 \mathrm{~nm}$ can be achieved using a newly developed fiber laser (MPBC, Montreal, Canada), which is very convenient due to its small size and price. Figure 3.19 shows cwSTED images of living cells labeled by the two approaches discussed here: The upper part shows PtK2 cells expressing Citrine targeted to the ER (green), whereas in the lower part, PtK2 cells expressing MAP2-hAGT are shown, which were labeled with CP-TMR (red). Excitation was performed using cw lasers (both Dual Calypso, Cobolt, Solna, Sweden) emitting at $491 \mathrm{~nm}$ for Citrine and at $532 \mathrm{~nm}$ for TMR. As can be seen in both cases, a substantial increase in resolution can be obtained.

These examples prove that STED imaging in living cells is also viable when using $\mathrm{cw}$ lasers. A setup based on these lasers has several advantages for the routine application for imaging: besides the costs (the $592 \mathrm{~nm} \mathrm{cw}$ STED laser is about only 10\% the price of the pulsed Ti:Sapphire-OPO system required for getting STED light in this wavelength range), 
the system becomes easier to handle, because these lasers require less alignment than the OPO does. Furthermore, with the omission of the timing between excitation and STED pulses, another step of alignment becomes obsolete. Therefore, a rather cheap, easy to handle and compact system for high-resolution STED imaging in living cells can be built by adding just a few parts to a conventional confocal microscope, giving rise to widespread applicability.
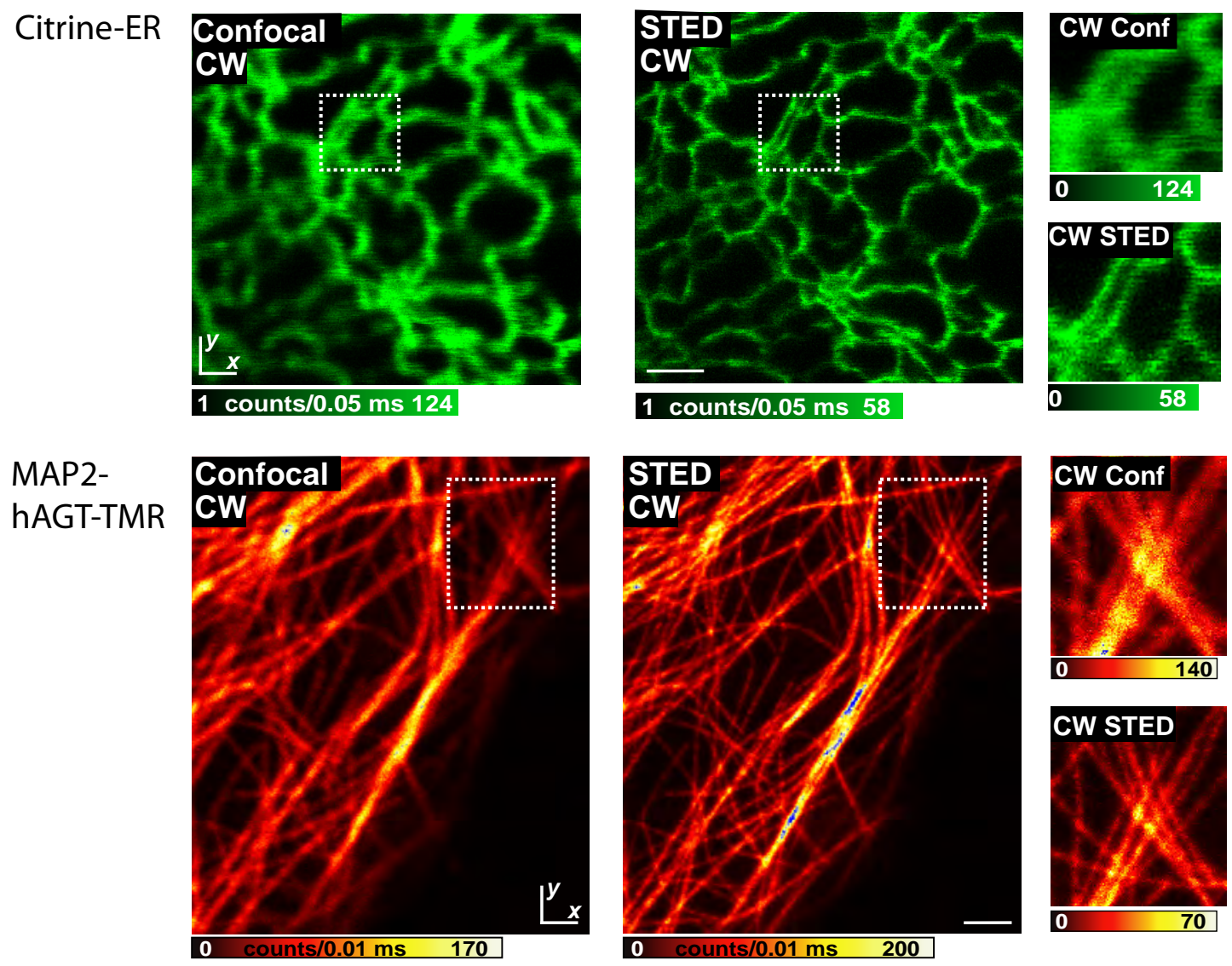

Figure 3.19: Live cell STED imaging using cw lasers. Upper part: Cells expressing Citrine targeted to the endoplasmic reticulum were imaged in the confocal and the STED mode by using cw lasers emitting at $488 \mathrm{~nm}$ for excitation and a powerful $\mathrm{cw}$ fiber laser operating at $592 \mathrm{~nm}$ for the STED beam. A similar resolution increase as in the case of using a pulsed laser system can be obtained. Lower part: A Krypton-laser can be employed as the STED laser for imaging living cells expressing TMR-labeled hAGT fusion proteins. Here, MAP2 in living PtK2 cells was imaged. The STED image shows again a similar gain in resolution as was demonstrated in the last paragraphs by using pulsed lasers. Scale bars $1 \mu \mathrm{m}$. 


\subsection{The Adequate Objective Lens}

A crucial factor in imaging is the choice of the appropriate objective lens. In addition to the numerical aperture, the immersion medium for which the objective is corrected for is an important factor. So far, all images presented here were acquired using a 1.4-NA oil immersion lens.

As opposed to that, the use of 1.2-NA water lenses would have provided an even deeper penetration depth along the $\mathrm{z}$ axis, because the matching of the refractive index of the immersion liquid with that of the medium of the cell avoids spherical aberration. Likewise, because the semiaperture angle of a 1.4-NA oil immersion objective is $\alpha=68^{\circ}$ and, therefore, slightly larger than that of a similar water immersion objective $\left(\alpha=64^{\circ}\right)$, some of the laser light is lost because of total internal reflection on the interface between the cover glass and the aqueous medium. On the other hand, the number of collected photons is larger with the oil immersion lens because of the larger collection angle.

In the cases presented here, spherical aberrations resulting from mismatch of the refractive index were minor because the imaged structures were relatively close (a few micrometers) to the coverslip. However, for imaging deeper in tissue, the usage of other objectives is advantageous. Figure 3.20 shows an example where a water-dipping objective was deployed to image a living PtK2 cell expressing Citrine targeted to the ER. Comparison with figure 3.2 shows that similar resolutions can be obtained as with the oil immersion objective. Therefore, live cell STED imaging with a deep penetration depth becomes also feasible.
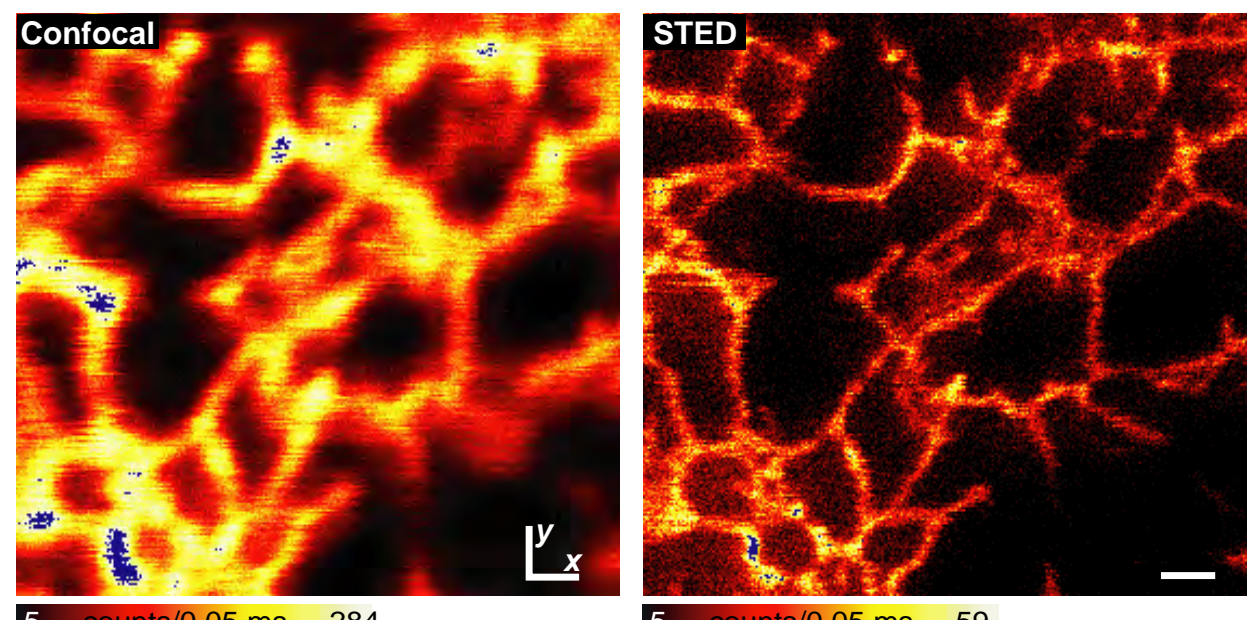

Figure 3.20: Citrine-labeled endoplasmic reticulum of a living mammalian cell imaged both in confocal (left) and STED (right) mode by using a water dipping objective. Scale bar $500 \mathrm{~nm}$. 


\subsection{Comparison of the Labeling Approaches: Which One to Choose for Live-Cell Nanoscopy?}

In this chapter, two different approaches for live cell STED imaging were presented: autofluorescent proteins as well as tagging proteins binding organic dyes. This raises the question as to which approach one should use for further experiments in living samples.

Figure 3.21 shows images of fluorescently labeled vimentin within living PtK2 cells. In the upper part (red), the fusion partner was hAGT, which was then labeled with TMR. The lower part shows the same structure, where Citrine was fused to Vimentin as a fluorescence label.
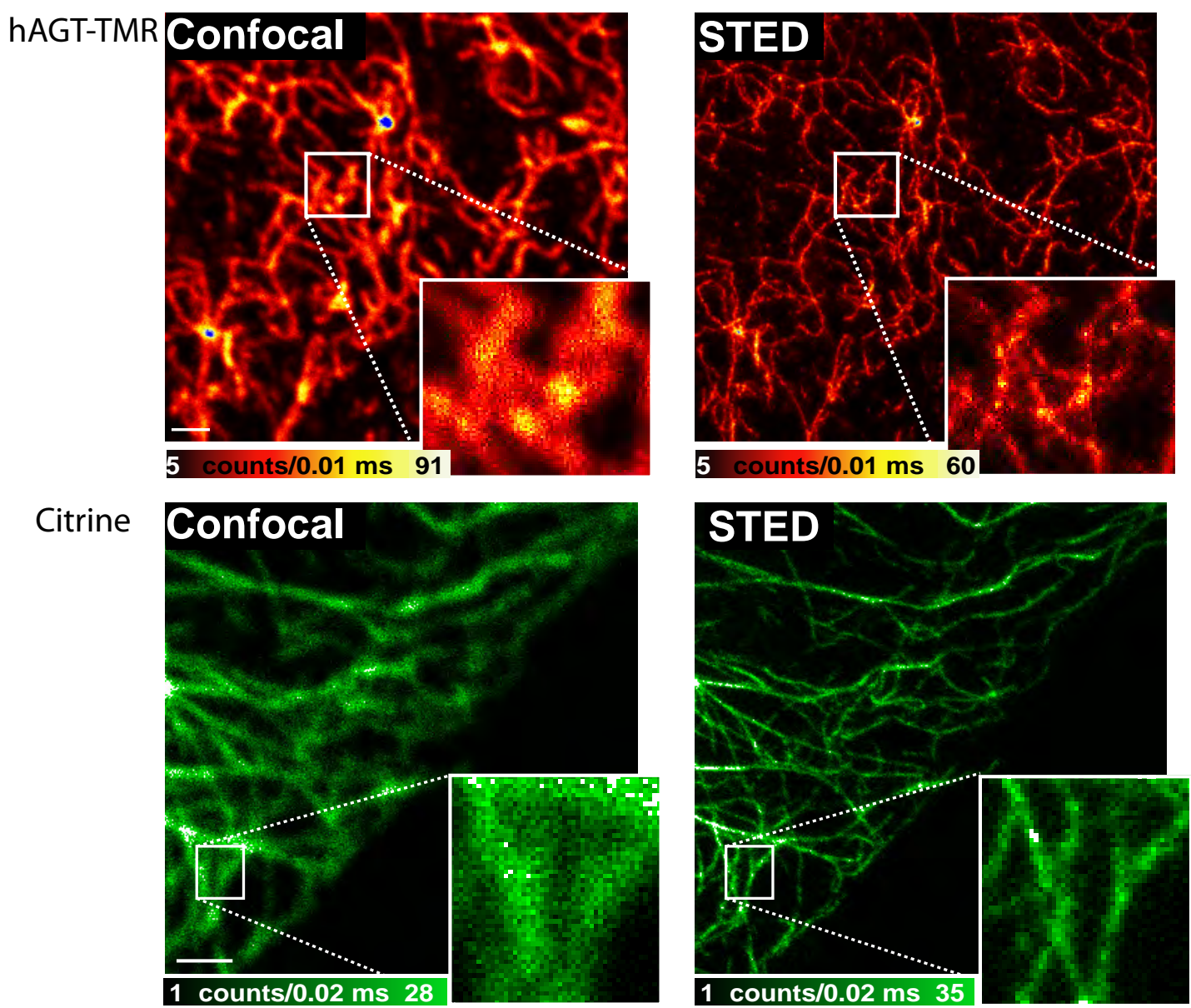

Figure 3.21: Vimentin in living PtK2 cells, labeled either with the tagging protein hAGT and TMR as the fluorophore (upper part) or the autofluorescent protein Citrine (lower part). In both cases, STED imaging reveals the structure in more detail. Scale bars $1 \mu \mathrm{m}$. 
In both cases, a significant resolution increase by STED microscopy is visible. Also, the structure looks similar in both cases, which is not the case when using HaloTag as a fusion partner (compare figure 3.18). This can also be verified by fusing both Citrine and hAGT to caveolin, as shown in figure 3.22. Caveolins are a family of membrane proteins involved in receptor independent endocytosis [68]. Here again, similar structures for both tagging approaches can be seen. Since the molecular size of hAGT and autofluorescent, monomeric proteins is similar (unlike HaloTag, which is substantially bigger), it is not surprising that if a fusion is successful with one protein, it also works with the other. Nevertheless, careful examination of fusion protein induced disturbances is necessary for every fusion partner.
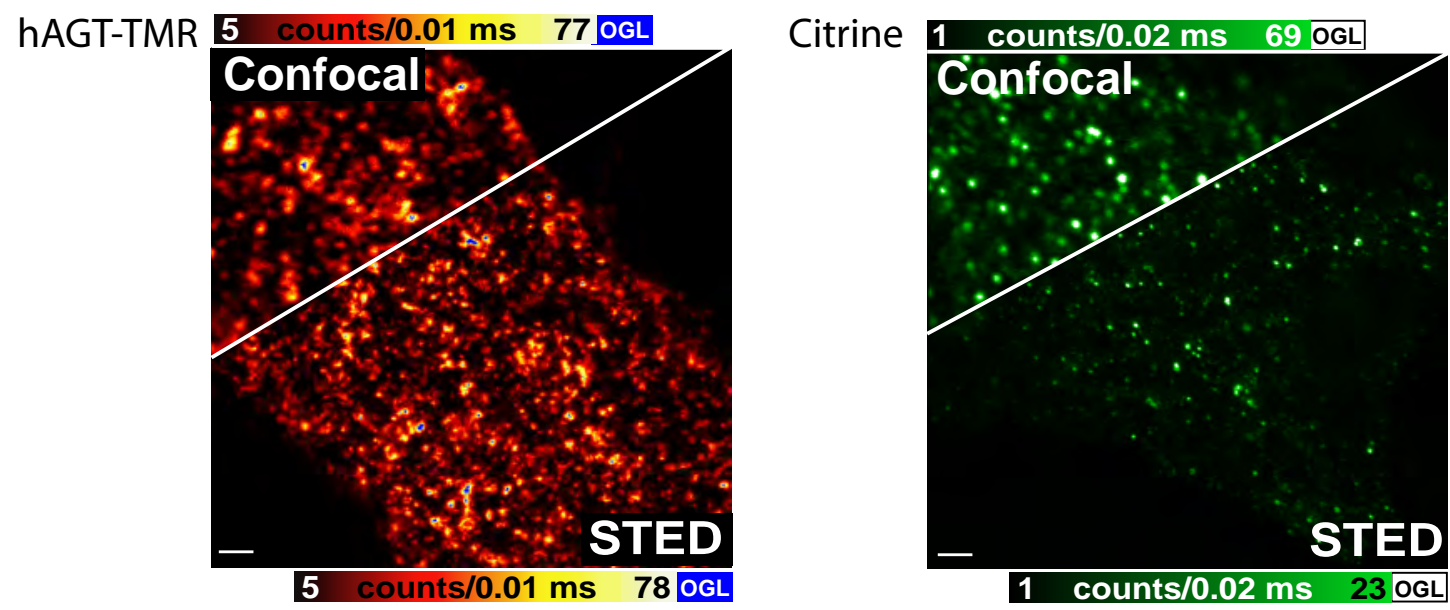

Figure 3.22: STED imaging of Caveolin labeled either with hAGT-TMR (right) and Citrine (left) results in similar images for both labeling approaches. Scale bars $1 \mu \mathrm{m}$.

As both examples presented here depict, a similar resolution enhancement can be achieved using these two approaches. In this work, several examples have been shown for both approaches where structural changes or movements within living cells were monitored with subdiffractive resolution. However, as in any microscopy method, the length and resolution of these nanoscopic videos is limited by the photostability of the fluorophore.

Comparisons of Citrine or other fluorescent proteins with many organic dyes coupled to an antibody show that - like in confocal microscopy - photobleaching is stronger in the first case. Therefore, it was assumed that by using organic dyes as a label for live cell imaging, samples as bright and photostable as when working with immunocytochemistry can be obtained. Two examples discussed here show the specific labeling in living cells using organic dyes. However, it has to be considered that in this case only a single fluorophore is attached per protein. When working with antibodies, each antibody is decorated with several fluorophores. By using a system where a primary antibody recognizes the structure of interest, and a secondary antibody binds the first one, this signal amplification gets even potentiated, because often more than one secondary antibody binds the first one. This gives rise to a very strong signal, thereby reducing the required exposition time. Quenching effects 
can also be observed in the latter case, which lead to a delay in fluorescence signal decrease upon photobleaching: If initially quenching between closely spaced fluorophores onto a single antibody takes place, bleaching of the emitter does not result in a signal decrease, because now the formerly quenched fluorophore can fluoresce.

This effect does not take place in the live cell labeling approaches discussed here; per protein, at most a single fluorophore is present. Therefore, it is not surprising that bleaching of organic dyes attached to a tagging protein is in the same order of magnitude as bleaching of autofluorescent proteins.

A direct comparison of these two methods is difficult because of the large variances in living samples. However, the general impression is that by using TMR as a label for hAGT, more images and a better resolution with similar structures could be obtained. The performance of Oregon Green is worse than of TMR, which is not surprising, because Oregon Green as a fluorescein derivative is known to posses a rather low photostability.

The main advantage of hAGT fusions, however, is the easy modification of the fluorophore. In addition to using more photostable dyes than the ones used in this work, one could also synthesize a construct where two or even more fluorophores are attached to the BG-linker; therefore, upon binding the linker, the protein would be labeled with several fluorophores. This would give rise to a signal amplification like when using antibodies. In the case of autofluorescent proteins, attaching more fluorophores to a protein of interest would require the attachment of several autofluorescent proteins, leading to an even bigger tag which is more likely to interfere with the structure of the protein of interest.

Separately, many experiments would benefit from a reduction in size of the tagging protein. As discussed before, there are very small tags which can be modified with organic dyes; if further developments achieve the intracellular use of these proteins while using photostable labels, this will be an important advantage.

On the other hand, when working with proteins where an organic dye has to be attached, additional labeling and washing steps are necessary. In addition to background due to nonspecific binding, it also has to be ensured that the modified fluorophore is not instantaneously transported out of the cell, which is for example often a problem in yeast cells. Also, for imaging in tissue or whole animals, accessibility of the fluorophore to the location of interest might be an issue.

All these factors have to be taken into account when deciding which labeling system to use.

With further improvements both in fluorescent protein engineering and organic dye synthesis as well as advancements in the tagging proteins in store, it remains to be seen where the more important progress for STED microscopy will be achieved. Thus far, both methods work well, with a slight lead for the TMR-hAGT tagging approach, as long as biology permits. 


\section{Live-Cell STED FCS: Fluorescence Correlation Spectroscopy in Reduced Focal Volumes}

For many cell biological experiments, information not only about structures, but also regarding the dynamics within the structure is of great significance. One approach to get information about the dynamics is to use Fluorescence Correlation Spectroscopy (FCS). Here, fluctuations in the fluorescence intensity caused by single molecules are measured and analyzed, which - amongst others - reveals information about the molecular diffusion behavior. It combines the advantages of fluorescence methods (like the noninvasive nature and the rather high spatial resolution) with a high temporal resolution and has therefore become an important tool in the life sciences.

The concept of FCS was introduced by D. Magde, E. Elson and W. W. Webb in the early 1970s, who successfully measured the diffusion and chemical dynamics of drugs intercalating DNA [69] [70]. With the subsequent development of powerful and stable light sources (lasers), bright and photostable fluorophores and more sensitive detectors (singlephoton counting APDs), the major weaknesses of this method were overcome. Another quantum leap was the background suppression by using a confocal microscope setup for doing FCS by R. Rigler et al. in the early 1990s, which now has become the standard setup for FCS measurements [71] [72].

In an FCS experiment, the changes in the fluorescence intensity from single fluorophores over time are analyzed. Therefore, the sample is illuminated and the intensity trace over time is recorded. Changes in intensity can be due to alterations in the fluorescence capability of the fluorophore (like intermolecular or intramolecular reactions or by rearrangements in the microenvironment of the fluorophore) or due to diffusion of the molecules in and out of the detection volume. Since the intensity fluctuations detected from a molecule ensemble are smaller the more molecules reside within the excitation focus, FCS measurements have to be performed in rather sparsely labeled samples. Also, the spatial resolution is in the same way limited due to diffraction as confocal microscopy is.

Therefore, reducing the focal volume offers several advantages: More densely labeled samples can be investigated, and with a higher spatial resolution, small local differences in the substructure are distinguishable. Hence, the implementation of a STED microscope can improve FCS even further. Successful applications of STED FCS have already been shown [73] [74], but, so far, they were limited to artificial systems examining dyes diffusing in a solution, or to fluorescent lipid analogues incorporated into the plasma membrane of 
living cells. The labeling approaches discussed in the previous chapter for live-cell STED imaging allow for accessing protein dynamics within cells. Here, they are exemplarily used for investigating the diffusion of membrane-anchored proteins within the cell membrane by the means of STED FCS.

\subsection{Theoretical Model of FCS}

The theory of how to extract information from the measured intensity fluctuations is rather complex, but has been described in detail in several recent reviews [75] [76] [77]; therefore, here only a short overview will be given:

Fluctuations $\delta F(t)$ of the fluorescence signal $F(t)$ are defined as the deviations from the temporal average of the signal $\langle F(t)\rangle$ :

$$
\delta F(t)=F(t)-\langle F(t)\rangle
$$

The fluorescence intensity $F(t)$ detected is the sum of the contributions from all volume elements within the detection volume $V$ :

$$
F(t)=\sigma \cdot q \cdot \kappa \cdot \int_{V} I_{e}(r) \cdot h_{d}(r) \cdot C(r, t) d^{3} r
$$

The fluorophore-specific parameters $\sigma$ and $q$ denote the absorption cross section and the fluorescence quantum yield, respectively. $\kappa$ represents the overall detection efficiency of the setup. $C(r)$ is the concentration of the fluorophore at the position $r=(x, y, z), I_{e}$ the spatial distribution of the excitation energy, which is related to the maximal amplitude $I_{0}$ by $I_{e}=h_{e} \cdot I_{0}$. For simplicity, a linear dependence on the excitation intensity is assumed here; for high intensities, deviations from linearity have to be considered. $h_{D}$ is the normalized probability distribution function for detecting a photon emitted at $r$.

The latter two distribution functions are often combined into a single dimensionless brightness function $B(r)=h_{e}(r) h_{d}(r)$, which is frequently approximated by a three-dimensional Gaussian profile:

$$
B(r(x, y, z))=\exp \left(-2\left[x^{2}+y^{2}\right] / r_{0}^{2}\right) \exp \left(-2 z^{2} / z_{0}^{2}\right)
$$

$r_{0}$ and $z_{0}$ denote the lateral and axial coordinates, respectively, at which the intensity has dropped from its initial value to a factor of $e^{-2}$.

The remaining parameters $\kappa, \sigma$ and $q$ can be combined together with the excitation intensity amplitude $I_{0}$ to give a measure of the photon count rate per detected molecule per second:

$$
\eta=I_{0} \cdot \kappa \cdot \sigma \cdot q
$$

Therefore, changes in fluorescence intensity can be described as

$$
\delta F(t)=\int_{V} B(r) \delta(\eta \cdot C(r, t)) \cdot d V
$$


These fluctuations are now analyzed by the normalized second-order autocorrelation function, which is defined as

$$
\begin{aligned}
G(\tau) & =\frac{<F(t) F(t+\tau)>}{<F(t)>^{2}} \\
& =1+\frac{<\delta F(t) \delta F(t+\tau)>}{<F(t)>^{2}} \\
& =1+\frac{<\delta F(0) \cdot \delta F(\tau)>}{<F^{2}>}
\end{aligned}
$$

Substitution of 4.4 into 4.5 results in

$$
G(\tau)=\frac{\iint B(r) B\left(r^{\prime}\right)\left\langle\delta(\eta \cdot C(r, t)) \delta\left(\eta \cdot C\left(r^{\prime}, t+\tau\right)\right)\right\rangle d V d V^{\prime}}{\left(\int B(r)\langle\delta(\eta \cdot C(r, t))\rangle d V\right)^{2}}
$$

If it is now assumed that the photophysical properties of the fluorophore do not change in the observable time regime, meaning that the measured intensity fluctuations are only due to diffusion of the fluorophores into and out of the detection volume, equation 4.6 is simplified to

$$
G(\tau)=\frac{\iint B(r) B\left(r^{\prime}\right)\left\langle\delta C(r, 0) \delta C\left(r^{\prime}, \tau\right)\right\rangle d V d V^{\prime}}{\left(\langle C\rangle \int B(r) d V\right)^{2}}
$$

Considering now only particles freely diffusing in three dimensions with the diffusion coefficient $D$, the so-called number density autocorrelation term $\left\langle\delta C(r, 0) \delta C\left(r^{\prime}, \tau\right)\right\rangle$ can be calculated by

$$
\left\langle\delta C(r, 0) \delta C\left(r^{\prime}, \tau\right)\right\rangle=\langle C\rangle(4 \pi D \tau)^{3 / 2} \cdot e^{-\frac{\left(r-r^{\prime}\right)^{2}}{4 D \tau}}
$$

This equation can be converted into a more commonly used form by the following two dependencies: Firstly, the relationship between the diffusion time $\tau_{D}$ that a molecule stays in the focal volume, and the diffusion coefficient $D$ is given by

$$
\tau_{D}=\frac{r_{0}^{2}}{4 \cdot D}
$$

The effective volume, which is considered to be Gaussian-shaped, can be calculated as

$$
V_{e f f}=\pi^{\frac{3}{2}} \cdot r_{0}^{2} z_{0}
$$

With 4.9 and 4.10 , equation 4.7 now becomes the correlation for freely diffusing species

$$
G(\tau)=\frac{1}{V_{e f f}\langle C\rangle} \cdot \frac{1}{1+\tau / \tau_{D}} \cdot \frac{1}{\sqrt{1+\left(r_{0} / z_{0}\right)^{2} \cdot \tau / \tau_{D}}}
$$


For molecules diffusing not in three dimension, but being restricted to a two-dimensional surface, this reduces to [78]

$$
G_{2 D}(\tau)=\frac{1}{V_{e f f}\langle C\rangle} \cdot \frac{1}{1+\tau / \tau_{D}}
$$

In all the considerations thus far, free diffusion of the fluorophore was assumed; meaning that the mean square displacement increases linearly with the observation time. However, if there are obstacles in the diffusion pathway, the assumption of free diffusion is no longer valid. In complex systems like living cells, specific or nonspecific interactions with other molecules or cellular structures can account for deviations from free diffusion. In this case, the increase of the mean square displacement with time is no longer linear, but given by $\left\langle r^{2}\right\rangle \propto t^{\alpha}$ with the anomaly factor $\alpha<1$. One possible approach to deal with hindered diffusion is to modify the autocorrelation function by taking this anomality factor $\alpha$ into account [79] [80]:

$$
G(\tau)=\frac{1}{V_{e f f}\langle C\rangle} \cdot \frac{1}{1+\left(\tau / \tau_{D}\right)^{\alpha}} \cdot \frac{1}{\sqrt{1+\left[\left(r_{0} / z_{0}\right)^{2} \cdot \tau / \tau_{D}\right]^{\alpha}}}
$$

Depending on the fluorophore used, fluctuations in the fluorescence intensity are not only due to diffusion, but also to changes in the brightness of the fluorophore. In that case, these variations also have to be considered when analyzing the data. The most common reason for alterations in the brightness is the population of dark states like the triplet state, but other effects like changes in the absorption efficiency due to rotation can also be important. When taking these effects also into account, the total observable fluctuations can generally be analyzed by the product of the autocorrelation functions for each of the effects:

$$
G_{\text {total }}(\tau)=G_{\text {Diff }}(\tau) \cdot \prod G_{D y e}(\tau)
$$

\subsection{Combining FCS with STED}

As briefly discussed in the introduction of this chapter, one weakness in conventional FCS is the limited spatial resolution, allowing only for averaging of the dynamics over a diffractionlimited volume. By performing FCS measurements with a STED setup, smaller detection volumes are accessible. In addition to the gain in spatial resolution by this means, more densely labeled samples can also be analyzed.

Figure 4.1B shows the comparison of an FCS measurement of an organic dye (JA2) in water in the confocal mode (black curve) and the appropriate STED mode (red curve). As can clearly be seen from normalized raw data (left part), the reduced detection volume leads to a left-shift of the autocorrelation curve to shorter diffusion times $\tau_{D}$. 
A
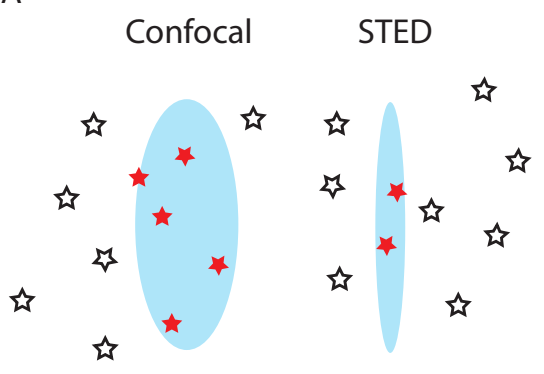

B
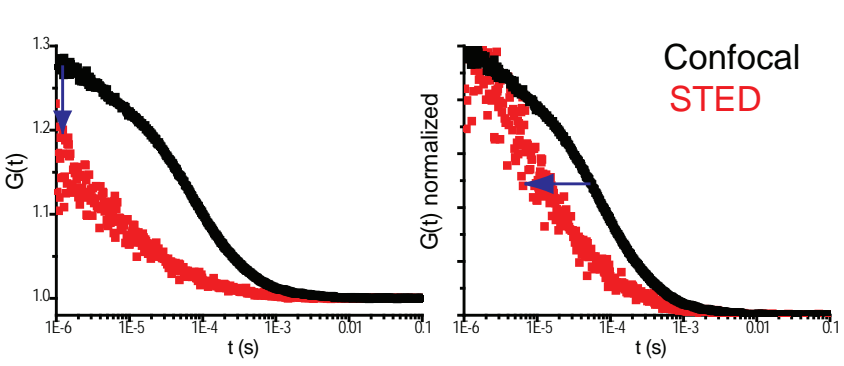

Figure 4.1: Principle of STED FCS: Fluctuations in fluorescence intensity due to diffusion of molecules in and out of the detection focus are detected. In STED FCS, the detection focus is decreased, enabling for higher spatial resolution (A). Autocorrelation curve of an organic dye (JA2) diffusing freely in solution. Uncorrelated background causes a decrease in the amplitude in STED experiments (B, left). The reduced detection volume in STED measurements leads to a decrease in the diffusion time, as can be seen from the normalized data (B, right).

One would also expect a decrease in the number of fluorophores. The number of fluorophores is - in the simple model described before - proportional to the reciprocal of the amplitude of the autocorrelation curve. However, as the raw data shows (figure 4.1 B, left), this is not the case here. This effect is well-known and has been attributed to uncorrelated background. A more complex model or an analysis using FIDA (Fluorescence Intensity Distribution Analysis) can account for the background, which therefore does not impede the experiments. The deviation of the STED focus from the Gaussian shape, which was used as a premise for the derivation of the utilized fitting model, has been shown to be of less importance.

In addition to what was discussed beforehand, photobleaching can be another cause for fluctuations in the fluorescence intensity, which leads to the incorrect assignment of shorter diffusion times. In STED FCS-measurements, however, the time the fluorophore spends in the detection volume is shortened compared to conventional FCS, therefore the probability of photobleaching is reduced. Photobleaching which may occur in the outer area of the excitation focus where the fluorophores are deexcited by the STED beam does not interfere with the measurements, because these molecules are not within the detection volume. 


\subsection{STED FCS in Living Cells}

In the previous chapter, different labeling approaches for live cell STED imaging were introduced. These methods are also applicable for STED FCS measurements in living cells, because the demands on the fluorophore are similar.

So far, STED FCS in biological systems was limited to synthetic lipids which were covalently labeled with an organic dye [81]. Lipid-exchange was used to incorporate them into the cell membrane. Although this is a versatile approach for studying lipid dynamics, protein dynamics could not be accessed by this means. By using the approaches discussed in the previous chapter, the possible fields of application of STED FCS will be greatly expanded.

In this chapter, the applicability of performing STED FCS using genetically encoded tags will be exemplarily demonstrated by monitoring the dynamics of a membrane-anchored protein.

\subsubsection{Dynamics of GPI-anchored Proteins}

Many proteins located in the outer cell membrane are anchored within the membrane via a glycosylphosphatidylinositol (GPI) anchor. The GPI anchor is a glycolipid attached to the Cterminus of a protein by a posttranslational modification. Two fatty acids of the hydrophobic phosphatidylinositol group are inserted into the lipid bilayer, thereby anchoring the protein in the outer cell membrane [82].

GPI anchored proteins are thought to associate with so-called lipid rafts, which are special membrane microdomains enriched in cholesterol and sphingolipids [83] [84]. Direct observation of these rafts, however, has been difficult, because they are highly dynamic and their size is well below the diffraction limit [85] [86]. Conventional light microscopic techniques therefore only average over several densely packed raft domains. Increasing the resolution using STED microscopy overcomes this limitation by giving the option to arbitrarily decrease the detection volume.

For addressing the dynamics of GPI-anchored proteins, fusion proteins with Citrine, ACP and hAGT (see chapter 3) were prepared and, in the latter two cases, labeled with different organic fluorophores. Cells expressing these fusion proteins were examined using a STED microscope: The excitation focus was targeted directly onto the fluorescent cell membrane. The fluorescence signal trace over time was recorded, from which then parameters characterizing the dynamics were extracted as described in the previous paragraphs. By adjusting the intensity of the STED beam, the size of the effectively exciting focus was tuned. Its dimensions were estimated by experimentally determining $I_{\text {sat }}$ for the fluorophores used and calculating the effective PSF for given STED intensities.

Figure 4.2 shows the dynamic behavior of a GPI-anchored hAGT fusion protein expressed in PtK2 cells labeled with Atto565: From the fluorescence trace, an autocorrelation curve was calculated for both confocal and STED mode (A). Diffusion in the membrane is limited to two dimensions; hence, a model based on equation 4.12 was employed for fitting the data. A simple fit with a single diffusion time did not describe the data with a reduced focal volume. Instead, a model accounting for anomalous diffusion as depicted by equation 
A

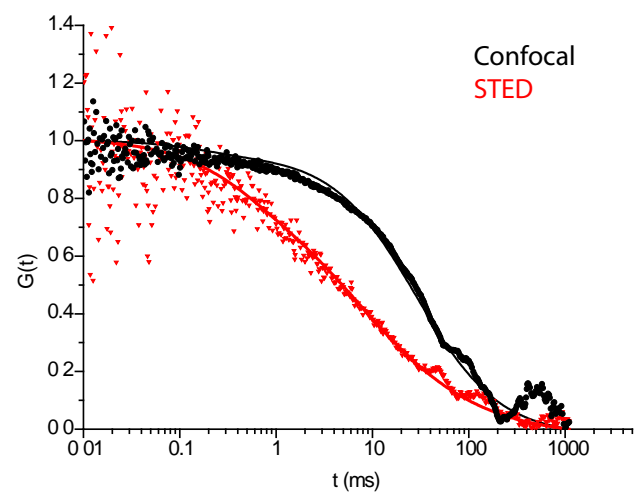

B

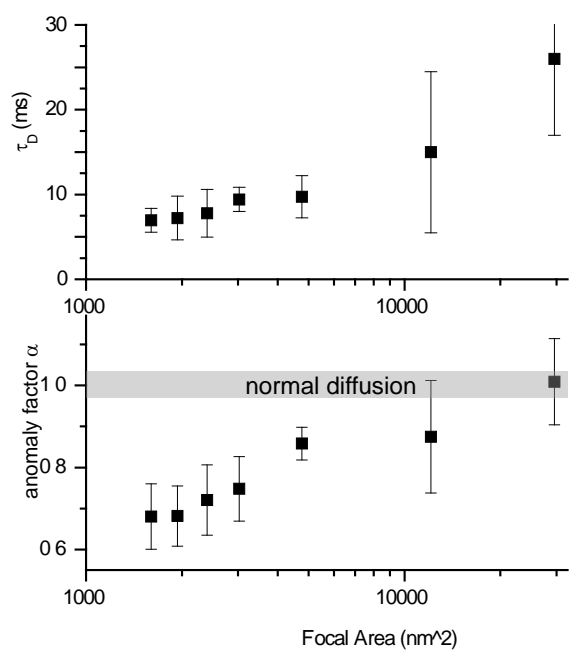

Figure 4.2: Dynamics of a GPI-anchored protein labeled with hAGT-Atto565 in a living PtK2 cell. A: Normalized autocorrelation curves; raw data and fit using a model considering anomalous diffusion in two dimensions. For different STED intensities, corresponding to different detection volumes, observation time $\tau_{D}$ and anomaly-factor $\alpha$ were calculated (B). For small detection volumes well below the diffraction limit, the dependency of the diffusion time to the detection volume is no longer linear, meaning hindered diffusion is observed. In agreement with that, the anomaly-factor $\alpha$ deviates from 1 for small detection volumes, which implies anomalous diffusion in subdiffraction areas as well.

4.13 had to be employed. Comparison of the (normalized) autocorrelation function with the fit as exemplified in figure 4.2 A indicate the validity of this assumption, because both the confocal and the STED autocorrelation function are well described.

For different STED intensities - corresponding to different detection areas - fluorescence intensity traces were recorded and autocorrelation curves were calculated. Figure 4.2B shows the dependence of the transit time $\tau_{D}$ and the anomaly-factor $\alpha$ on the detection volume: As expected, $\tau_{D}$ decreases with decreasing detection volume. However, this dependence is not linear when going to small detection areas, as expected for freely diffusing species. In consistency with the fact that the confocal data in figure 4.2 $\mathrm{B}$ could be also fitted with a single diffusion time, the anomaly-factor for confocal detection is around 1, therefore no anomaly is present. However, for reduced detection volumes, $\alpha$ decreases, meaning that heterogeneous diffusion is observed. This heterogeneity can be attributed to the GPI-anchor being trapped for a short time in some nanodomains or molecular complexes on a tiny spatial scale before normal diffusion continues. Similar interactions have been shown for fluorescent sphingolipid analogues before [81]. In contrast, normal, homogeneous diffusion has been demonstrated for fluorescent phospholipid analogues. This confirms the hypothesis that GPIanchored proteins temporarily associate with molecular complexes on the nanoscale like lipid rafts. 
To further see whether this trapping is effected by lipid raft like interactions, the influence of cholesterol has to be examined: Lipid rafts are enriched in cholesterol, therefore, a cholesterol depletion will lead to changes in the dynamic behavior, if it is influenced by the presence of these rafts. Preliminary results using cholesterol oxidase as a depleting agent indicate a change of the GPI-anchor dynamics. However, as discussed in the literature, cholesterol depletion may also lead to the formation of solid-like regions in the plasma region [81]. Therefore, more careful experiments are needed to prove that trapping of GPI-anchored proteins is due to cholesterol-enriched phases.

Nevertheless, the experiments presented here show that trapping on the nanoscale takes place. Since this effect is only visible in subdiffraction detection volumes, STED FCS offers a unique possibility to monitor this behavior.

\subsubsection{Influence of the Label}

To rule out that the trapping is induced by the label, other fluorophores and genetically encoded tags were applied. The excitation and STED wavelengths were optimized for the fluorophore; otherwise, FCS measurements were performed in the same way as described for Atto565-labeled hAGT fusions. The STED intensity was adjusted to achieve an effective spot of excitation of about $65 \mathrm{~nm}$ in the STED measurements for each fluorophore.

A comparison of the anomaly factor $\alpha$ for both confocal and STED FCS measurements is shown in figure 4.3

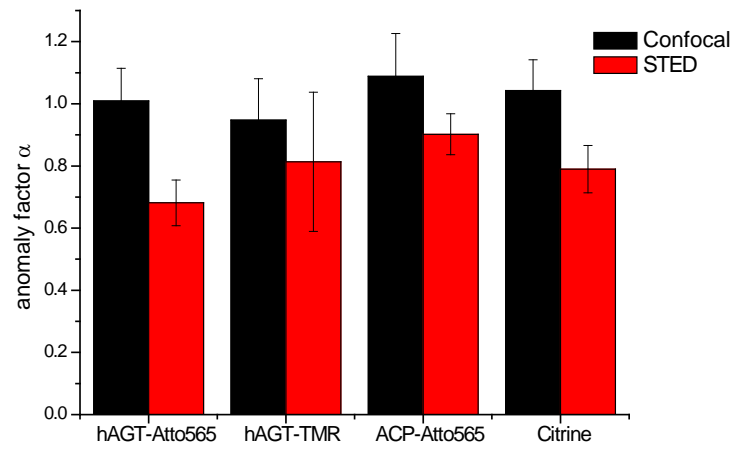

Figure 4.3: For different labeling approaches, hindered diffusion of GPI-anchored proteins can be observed in subdiffraction detection volumes only.

In addition to labeling hAGT with Atto565, tetramethylrhodamine (TMR) was used. Because this is a membrane-permeable substrate, dynamics in the interior of the cell are also accessible. Similar results for the dynamics of the GPI-anchor are obtained in this case: For large detection volumes (confocal), no anomaly is observed, whereas for decreased detection volumes, the anomaly-factor $\alpha$ deviates from 1, implying hindered diffusion. This consent is not surprising, because the fluorophore itself is small in comparison to the tagging protein; 
therefore, the influence of the fluorophore is expected to be marginal. However, due to the membrane permeability care has to be taken to exhaustively wash out unbound fluorophore from the cell body, which will otherwise lead to high background.

A different tagging-approach using acyl-carrier protein (ACP) was applied as well. As discussed in the previous chapter, ACP-labeling is limited to the extracellular space. In cases like the GPI-anchor, which is located in the outer cell membrane, this approach is advantageous, because the size of the ACP-tagging protein is smaller that the size of hAGT or GFP-like proteins. As can be seen, here a similar effect in the trapping behavior of the GPI-anchor is also observed.

The same holds true for using the yellow fluorescent protein Citrine; a similar trapping behavior is observed. However, free diffusion between two trapping events is faster than for all other approaches, leading to a smaller transit time of about $15 \mathrm{~ms}$ for confocal measurements, which is in good agreement with published data for EGFP-tagged GPI-anchor [87]. The cause of this discrepancy needs to be further investigated. It shows again, though, that the choice of the label can be decisive.

These examples demonstrate that using both autofluorescent proteins as well as tagging proteins binding organic dyes are a well-suited tool to study the dynamics of proteins within living cells with a resolution beyond the diffraction barrier. Due to the free choice of the substrate for ACP and hAGT when examining the outer cell membrane, the dye can be adapted to the experimental conditions like the STED microscope available. Also, twocolor experiments for FCCS (Fluorescence Cross-Correlation Spectroscopy) [88] in reduced detection volumes becomes feasible by these means. By using autofluorescent proteins or membrane-permeable substrates like TMR for hAGT, intracellular dynamics are accessible as well.

This greatly expands the possible fields of application of STED FCS, opening up new avenues to monitor dynamics in living cells on the nanoscale. 


\section{Conclusion and Outlook}

The superior resolving capacity of STED microscopy has already proved its exceptional potential in various biological experiments. However, so far mainly fixed and therefore dead samples could be investigated, leading to results which can only partially be assigned to the actually interesting system: the LIVING cell.

In this thesis, a general approach allowing for STED microscopy in living cells has been established. By this means, it is now possible to monitor structures and dynamics within living systems with an unprecedented resolution. This finally enables STED microscopy to be applied in an area where alternative methods offering a similar or even better resolution (like electron microscopy and near-field methods) fail.

Autofluorescent proteins derived from green fluorescent protein, which are commonly used in conventional live cell fluorescence imaging, were tested for their performance under STED conditions. The yellow fluorescent protein Citrine was shown to be the most stable under STED illumination. It was used for imaging different cytoskeletal structures (tubulin and vimentin), the endoplasmic reticulum, and protein clusters on the cell membrane with a lateral resolution down to $50 \mathrm{~nm}$ and an axial resolution of $150 \mathrm{~nm}$ both in mammalian cells and in yeast.

A versatile alternative to autofluorescent proteins are recently developed tagging proteins, which can selectively bind modified organic dyes. Different approaches have been used in this work: The ACP-tag allows for the labeling of structures on the cell membrane. For imaging intracellular structures, the tagging proteins hAGT, which is commercially available as SnapTag, and HaloTag were employed. Basically any organic dye can be used in conjunction with these tagging proteins; however, cell permeability has to be considered when addressing intracellular structures. Here, tetramethylrhodamine (TMR) and Oregon Green were used as membrane-permeable substrates, leading to a resolution down to $40 \mathrm{~nm}$ within living mammalian cells, as was demonstrated by imaging the cytoskeleton and different protein clusters on the cell membrane.

The possibility of live cell imaging also allows for monitoring structural changes within the cell over time. This requires the repeated imaging of the same area; therefore, the demand on the photostability of the fluorophore is higher than for only taking a single image, as is usually done when examining fixed samples. Nanoscopic videos showing structural changes of the ER - either spontaneous over time or induced by a chemical stimulus - were recorded using Citrine. The movement of connexin- 43 on the cell membrane was monitored with subdiffraction resolution using TMR to label hAGT. However, to record longer super- 
resolution movies (more than 10 to 20 frames), the photostability of the fluorophores needs to be improved.

The engineering of fluorescent proteins has already led to a number of variants optimized for different properties like photostability, sensitivity to certain small molecules or the ability to switch between different molecular states. A high-throughput-screening of randomly or rationally mutated proteins for enhanced stability under STED conditions may have similar success. Citrine as the most stable protein under STED illumination to date would be a good starting point for mutagenesis. The usage of tagging proteins binding organic fluorophores offers even more potential for improvement: In addition to employing more photostable organic dyes, other fluorophores like non-bleaching nitrogen-vacancies in nanodiamonds could be attached to the tagging protein. Alternatively, a substrate carrying more than a single fluorophore can be synthesized, leading to a multiplied brightness of the structure. An interesting alternative for dealing with photobleaching in a different way is the use of recently developed fluorogen enhancing peptides (FAPs) [89]: A cell expressing FAPs as a fusion protein is incubated with the appropriate fluorogen. The fluorogen becomes fluorescent only upon binding to the FAP, whereas in the unbound state, no fluorescence can occur due to fast radiationless decay of the excited state. Since the non-covalent interaction is rather weak, a fast exchange of FAP-bound molecules takes place. This replenishment of the potentially bleached fluorophore will allow for long-term imaging experiments using an excess of fluorogen. However, it remains to be seen whether these fluorogens are suitable fluorophores for STED microscopy.

Due to the free choice of fluorophore when using tagging proteins, live cell two-color imaging becomes feasible as well. By using a related tagging protein, which is commercially available as ClipTag (Covalys Biosciences, Switzerland) and which relies on a very similar mechanism as hAGT, different proteins can be simultaneously labeled with different fluorophores. Therefore, as was already shown for fixed samples, two-color live cell STED images can be obtained by using two fluorophores with distinct spectral properties.

Although with the beamscanning microscope employed in this work, protein dynamics can be followed with video-rate, different approaches have to be applied to examine even faster processes. By employing STED fluorescence correlation spectroscopy (STED FCS), it was exemplarily shown by using both autofluorescent proteins and tagging proteins that the diffusion of GPI-anchored proteins in the cell membrane is not free but hindered. This behavior can be explained by a transient trapping of the GPI-anchor in some complexes or membrane nanodomains. It can only be observed when squeezing down the detection volume to subdiffraction size, because conventional FCS averages over a comparably large area, not resolving single trapping events on the nanoscale. The observation of trapping on the nanoscale supports the hypothesis that the cell membrane is structured by molecular complexes or membrane nanodomains such as lipid rafts, which is subject of a vibrant scientific dispute. STED FCS so far was limited to artificial systems or lipid-dynamics within living cells; therefore, the usage of genetically encoded tags also here enables new insights on the nanoscale. 
The development of these STED compatible live cell labeling approaches can not only be used for examining living cells, but also tissue, plants and animals. First successful applications include the imaging of mouse brain slices [55] and the nematode Caenorhabditis elegans (unpublished data). However, the mismatch of refractive index calls for the usage of different objective lenses. In addition to the water dipping objective tested in this work, utilization of glycerine-objectives and, eventually, correcting for aberrations by adaptive optics will also facilitate imaging in deeper tissue.

For both yellow fluorescent proteins and hAGT labeled with TMR, in addition to the pulsed laser system consisting of a Ti:Sapphire laser and an optical parametric oscillator providing the STED light, cheaper and easy-to-handle continuous wave lasers were also employed. This not only decreases the cost of a microscope capable of live cell STED microscopy, it also facilitates the implementation of STED into conventional confocal microscopes.

To conclude, the outcome of this work enables live cell STED imaging, thereby finally exploiting one of the main advantages of this light microscopy technique, namely its compatibility with living cells. In combination with the future developments discussed here, the results presented will enable live cell STED microscopy to become a standard tool for imaging experiments in the life sciences, offering the potential of conventional fluorescence microscopy with an unprecedented resolution for live cell experiments. 


\section{Bibliography}

[1] Seneca. Quaestiones Naturalis.

[2] Abbe, E. Beiträge zur Theorie des Mikroskops und der mikroskopischen Wahrnehmung. Arch. Mikr. Anat., vol. 9, pp. 413-468, 1873.

[3] Stelluti, Francesco. Melissographia. 1625.

[4] Hooke, Robert. Micrographia. 1665.

[5] Minsky, M. Microscopy apparatus, US Patent 3,013,467, 1961.

[6] Wilson, T. Confocal microscopy. Academic Press Ltd., London, 1990.

[7] Pawley, J. Handbook of biological confocal microscopy. Plenum Press, New York, 1995.

[8] Paddock, S.W. Confocal microscopy. Humana Press, Totowa, NJ, USA, 1999.

[9] Synge, E. H. A suggested method for extending microscopic resolution into the ultramicroscopic region. Philosophical Magazine, vol. 6 (35), pp. 356-362, 1928.

[10] Dunn, R. C. Near-field scanning optical microscopy. Chemical Reviews, vol. 99 (10), pp. 2891-+, 1999.

[11] v. Borries, B. and Ruska, E. Electron microscope and its uses. Zeitschrift des Vereines Deutscher Ingenieure, pp. 519-524, 1935.

[12] Ruska, H. Supermicroscopy. Nederlands Tijdschrift voor Natuurkunde, pp. 179-191, 1940.

[13] Hell, S. W. Far-field optical nanoscopy. Science, vol. 316 (5828), pp. 1153-1158, 2007.

[14] Hell, S. W. Microscopy and its focal switch. Nature Methods, vol. 6 (1), pp. 24-32, 2009.

[15] Hell, S. W. and Wichmann, J. Breaking the diffraction resolution limit by stimulated emission: stimulated emission depletion microscopy. Opt. Lett., vol. 19 (11), pp. 780$782,1994$. 
[16] Klar, T.A. and Hell, S.W. Subdiffraction resolution in far-field fluorescence microscopy. Opt. Lett., vol. 24 (14), pp. 954-956, 1999.

[17] Schmidt, R., Wurm, C. A., Jakobs, S., Engelhardt, J., Egner, A., and Hell, S. W. Spherical nanosized focal spot unravels the interior of cells. Nature Methods, vol. 5 (6), pp. 539-544, 2008.

[18] Westphal, V. and Hell, S.W. Nanoscale Resolution in the Focal Plane of an Optical Microscope. Phys. Rev. Lett., vol. 94, p. 143903, 2005.

[19] Rittweger, E., Han, K. Y., Irvine, S. E., Eggeling, C., and Hell, S. W. STED microscopy reveals crystal colour centres with nanometric resolution. Nature Photonics, vol. 3 (3), pp. 144-147, 2009.

[20] Westphal, V., Rizzoli, S. O., Lauterbach, M. A., Kamin, D., Jahn, R., and Hell, S. W. Video-rate far-field optical nanoscopy dissects synaptic vesicle movement. Science, vol. 320 (5873), pp. 246-249, 2008.

[21] Harke, B., Ullal, C. K., Keller, J., and Hell, S. W. Three-dimensional nanoscopy of colloidal crystals. Nano Letters, vol. 8 (5), pp. 1309-1313, 2008.

[22] Donnert, G., Keller, J., Wurm, C. A., Rizzoli, S. O., Westphal, V., Schonle, A., Jahn, R., Jakobs, S., Eggeling, C., and Hell, S. W. Two-color far-field fluorescence nanoscopy. Biophysical Journal, vol. 92 (8), pp. L67-L69, 2007.

[23] Klar, T. A., Jakobs, S., Dyba, M., Egner, A., and Hell, S. W. Fluorescence microscopy with diffraction resolution limit broken by stimulated emission. Proc. Nat. Acad. Sci. U.S.A, vol. 97, pp. 8206-8210, 2000.

[24] Hell, S. W. and Kroug, M. Ground-state depletion fluorescence microscopy, a concept for breaking the diffraction resolution limit. Appl. Phys. B, vol. 60, pp. 495-497, 1995.

[25] Bretschneider, S., Eggeling, C., and Hell, S. W. Breaking the Diffraction Barrier in Fluorescence Microscopy by Optical Shelving. Phys. Rev. Lett., vol. 98, p. 218103, 2007.

[26] Hofmann, M., Eggeling, C., Jakobs, S., and Hell, S.W. Breaking the diffraction barrier in fluorescence microscopy at low light intensities by using reversibly photoswitchable proteins. Proc. Natl. Acad. Sci. USA, vol. 102 (49), pp. 17565-17569, 2005.

[27] Bossi, M., Fölling, J., Dyba, M., Westphal, V., and Hell, S. W. Breaking the diffraction resolution barrier in far-field microscopy by molecular optical bistability. New J. Phys., vol. 8, p. 275, 2006.

[28] Kural, C., Kim, H., Syed, S., Goshima, G., Gelfand, V. I., and Selvin, P. R. Kinesin and dynein move a peroxisome in vivo: A tug-of-war or coordinated movement? Science, vol. 308 (5727), pp. 1469-1472, 2005. 
[29] Heisenberg, W. The physical principles of the quantum theory. Chicago Univ. Press, Chicago, 1930.

[30] Betzig, E., Patterson, G.H., Sougrat, R., Lindwasser, O.W., Olenych, S., Bonifacino, J.S., Davidson, M.W., Lippincott-Schwartz, J., and Hess, H.F. Imaging Intracellular Fluorescent Proteins at Nanometer Resolution. Science, vol. 313 (5793), pp. 16421645, 2006.

[31] Hess, Samuel T., Girirajan, Thanu P. K., and Mason, Michael D. Ultra-High Resolution Imaging by Fluorescence Photoactivation Localization Microscopy. Biophys. J., vol. 91 (11), pp. 4258-4272, 2006.

[32] Rust, Michael J., Bates, Mark, and Zhuang, Xiaowei. Sub-diffraction-limit imaging by stochastic optical reconstruction microscopy (STORM). Nat. Methods, vol. 3, pp. 793-796, 2006.

[33] Fölling, J., Bossi, M., Bock, H., Medda, R., Wurm, C. A., Hein, B., Jakobs, S., Eggeling, C., and Hell, S. W. Fluorescence nanoscopy by ground-state depletion and single-molecule return. Nature Methods, vol. 5 (11), pp. 943-945, 2008.

[34] Alberts, B., Johnson, A., Lewis, J., Raff, M., Roberts, K., and Walter, P. Molecular Biology of the Cell. Garland Science, New York, 4 edition, 2002.

[35] Tsien, Roger Y. The green fluorescent protein. Annu. Rev. Biochem., vol. 67 (1), pp. 509-544, 1998.

[36] Ormo, M., Cubitt, A. B., Kallio, K., Gross, L. A., Tsien, R. Y., and Remington, S. J. Crystal structure of the Aequorea victoria green fluorescent protein. Science, vol. 273 (5280), pp. 1392-1395, 1996.

[37] Shaner, N. C., Steinbach, P. A., and Tsien, R. Y. A guide to choosing fluorescent proteins. Nature Methods, vol. 2 (12), pp. 905-909, 2005.

[38] O'Hare, H. M., Johnsson, K., and Gautier, A. Chemical probes shed light on protein function. Current Opinion in Structural Biology, vol. 17 (4), pp. 488-494, 2007.

[39] Griffin, B. A., Adams, S. R., and Tsien, R. Y. Specific covalent labeling of recombinant protein molecules inside live cells. Science, vol. 281 (5374), pp. 269-272, 1998.

[40] Martin, B. R., Giepmans, B. N. G., Adams, S. R., and Tsien, R. Y. Mammalian cellbased optimization of the biarsenical-binding tetracysteine motiffor improved fluorescence and affinity. Nature Biotechnology, vol. 23 (10), pp. 1308-1314, 2005.

[41] Lata, S., Gavutis, M., Tampe, R., and Piehler, J. Specific and stable fluorescence labeling of histidine-tagged proteins for dissecting multi-protein complex formation. Journal of the American Chemical Society, vol. 128 (7), pp. 2365-2372, 2006. 
[42] Miller, L. W., Cai, Y. F., Sheetz, M. P., and Cornish, V. W. In vivo protein labeling with trimethoprim conjugates: a flexible chemical tag. Nature Methods, vol. 2 (4), pp. 255-257, 2005.

[43] George, N., Pick, H., Vogel, H., Johnsson, N., and Johnsson, K. Specific labeling of cell surface proteins with chemically diverse compounds. Journal of the American Chemical Society, vol. 126 (29), pp. 8896-8897, 2004.

[44] Keppler, A., Gendreizig, S., Gronemeyer, T., Pick, H., Vogel, H., and Johnsson, K. A general method for the covalent labeling of fusion proteins with small molecules in vivo. Nature Biotechnology, vol. 21 (1), pp. 86-89, 2003.

[45] Keppler, A., Pick, H., Arrivoli, C., Vogel, H., and Johnsson, K. Labeling of fusion proteins with synthetic fluorophores in live cells. Proceedings of the National Academy of Sciences of the United States of America, vol. 101 (27), pp. 9955-9959, 2004.

[46] Lamesch, P., Li, N., Milstein, S., Fan, C. Y., Hao, T., Szabo, G., Hu, Z. J., Venkatesan, K., Bethel, G., Martin, P., Rogers, J., Lawlor, S., McLaren, S., Dricot, A., Borick, H., Cusick, M. E., Vandenhaute, J., Dunham, I., Hill, D. E., and Vidal, M. hORFeome v3.1: A resource of human open reading frames representing over 10,000 human genes. Genomics, vol. 89 (3), pp. 307-315, 2007.

[47] Wurm, C. A. and Jakobs, S. Differential protein distributions define two subcompartments of the mitochondrial inner membrane in yeast. Febs Letters, vol. 580 (24), pp. 5628-5634, 2006.

[48] Willig, K. I., Rizzoli, S. O., Westphal, V., Jahn, R., and Hell, S. W. STED-microscopy reveals that synaptotagmin remains clustered after synaptic vesicle exocytosis. Nature, vol. 440 (7086), pp. 935 - 939, 2006.

[49] Sieber, J. J., Willig, K. I., Kutzner, C., Gerding-Reimers, C., Harke, B., Donnert, G., Rammner, B., Eggeling, C., Hell, S. W., Grubmüller, H., and Lang, T. Anatomy and dynamics of a supramolecular membrane protein cluster. Science, vol. 317, pp. 1072 - 1076, 2007.

[50] Kittel, R. J., Wichmann, C., Rasse, T. M., Fouquet, W., Schmidt, M., Schmid, A., Wagh, D. A., Pawlu, C., Kellner, R., Willig, K. I., Hell, S. W., Buchner, E., Heckmann, M., and Sigrist, S. J. Bruchpilot Promotes Active Zone Assembly, Ca2+-Channel Clustering, and Vesicle Release. Science, vol. 312, pp. 1051-1054, 2006.

[51] Griesbeck, O., Baird, G. S., Campbell, R. E., Zacharias, D. A., and Tsien, R. Y. Reducing the environmental sensitivity of yellow fluorescent protein - Mechanism and applications. Journal of Biological Chemistry, vol. 276 (31), pp. 29188-29194, 2001.

[52] Cognet, L., Coussen, F., Choquet, D., and Lounis, B. Fluorescence microscopy of single autofluorescent proteins for cellular biology. Comptes Rendus Physique, vol. 3 (5), pp. 645-656, 2002. 
[53] Axelsson, M. A. B. and Warren, G. Rapid, endoplasmic reticulum-independent diffusion of the mitotic golgi haze. Molecular Biology of the Cell, vol. 15 (4), pp. 18431852, 2004.

[54] Takeichi, M. Cadherins - a Molecular Family Important in Selective Cell-Cell Adhesion. Annual Review of Biochemistry, vol. 59, pp. 237-252, 1990.

[55] Naegerl, U. Valentin, Willig, Katrin I., Hein, Birka, Hell, Stefan W., and Bonhoeffer, Tobias. Live-cell imaging of dendritic spines by STED microscopy. Proceedings of the National Academy of Sciences of the United States of America, vol. 105 (48), pp. 18982-18987, 2008.

[56] Nagai, T., Ibata, K., Park, E. S., Kubota, M., Mikoshiba, K., and Miyawaki, A. A variant of yellow fluorescent protein with fast and efficient maturation for cell-biological applications. Nature Biotechnology, vol. 20 (1), pp. 87-90, 2002.

[57] Ekman, A. C. and Leppaluoto, J. Neuropeptide Y: a new peptide regulating behavior. Duodecim, vol. 108 (1), pp. 17-24, 1992.

[58] Moreira, K. E., Walther, T. C., Aguilar, P. S., and Walter, P. Pill Controls Eisosome Biogenesis. Molecular Biology of the Cell, vol. 20 (3), pp. 809-818, 2009.

[59] Walther, T. C., Brickner, J. H., Aguilar, P. S., Bernales, S., and Walter, P. Eisosomes mark static sites of endocytosis. Nature, vol. 439 (7079), pp. 998-1003, 2006.

[60] Grossmann, G., Malinsky, J., Stahlschmidt, W., Loibl, M., Weig-Meckl, I., Frommer, W. B., Opekarova, M., and Tanner, W. Plasma membrane microdomains regulate turnover of transport proteins in yeast. Journal of Cell Biology, vol. 183 (6), pp. 10751088, 2008.

[61] Heinlein, T., Knemeyer, J. P., Piestert, O., and Sauer, M. Photoinduced electron transfer between fluorescent dyes and guanosine residues in DNA-hairpins. Journal of Physical Chemistry B, vol. 107 (31), pp. 7957-7964, 2003.

[62] Goodenough, D. A., Goliger, J. A., and Paul, D. L. Connexins, connexons, and intercellular communication. Annual Review of Biochemistry, vol. 65, pp. 475-502, 1996.

[63] Illenberger, S., Drewes, G., Trinczek, B., Biernat, J., Meyer, H. E., Olmsted, J. B., Mandelkow, E. M., and Mandelkow, E. Phosphorylation of microtubule-associated proteins MAP2 and MAP4 by the protein kinase p110(mark) - Phosphorylation sites and regulation of microtubule dynamics. Journal of Biological Chemistry, vol. 271 (18), pp. 10834-10843, 1996.

[64] Dehmelt, L. and Halpain, S. The MAP2/Tau family of microtubule-associated proteins. Genome Biology, vol. 6 (1), 2005. 
[65] Los, G. V., Encell, L. P., McDougall, M. G., Hartzell, D. D., Karassina, N., Zimprich, C., Wood, M. G., Learish, R., Ohane, R. F., Urh, M., Simpson, D., Mendez, J., Zimmerman, K., Otto, P., Vidugiris, G., Zhu, J., Darzins, A., Klaubert, D. H., Bulleit, R. F., and Wood, K. V. HatoTag: A novel protein labeling technology for cell imaging and protein analysis. Acs Chemical Biology, vol. 3 (6), pp. 373-382, 2008.

[66] Wildanger, D., Rittweger, E., Kastrup, L., and Hell, S. W. STED microscopy with a supercontinuum laser source. Optics Express, vol. 16 (13), pp. 9614-9621, 2008.

[67] Willig, K. I., Harke, B., Medda, R., and Hell, S. W. STED microscopy with continuous wave beams. Nature Methods, vol. 4 (11), pp. 915-918, 2007.

[68] Williams, T. M. and Lisanti, M. P. The caveolin proteins. Genome Biology, vol. 5 (3), 2004.

[69] Elson, E. L. and Magde, D. Fluorescence Correlation Spectroscopy .1. Conceptual Basis and Theory. Biopolymers, vol. 13 (1), pp. 1-27, 1974.

[70] Magde, D., Elson, E. L., and Webb, W. W. Fluorescence Correlation Spectroscopy .2. Experimental Realization. Biopolymers, vol. 13 (1), pp. 29-61, 1974.

[71] Rigler, R. and Widengren, J. Ultrasensitive detection of single molecules by fluorescence correlation spectroscopy. Lund University Press, Lund, 1990.

[72] Rigler, R., Mets, U., Widengren, J., and Kask, P. Fluorescence Correlation Spectroscopy with High Count Rate and Low-Background - Analysis of Translational Diffusion. European Biophysics Journal with Biophysics Letters, vol. 22 (3), pp. 169-175, 1993.

[73] Kastrup, L., Blom, H., Eggeling, C., and Hell, S. W. Fluorescence fluctuation spectroscopy in subdiffraction focal volumes. Physical Review Letters, vol. vol.94, no.17, pp. 178104/1-4, 2005.

[74] Blom, H., Kastrup, L., and Eggeling, C. Fluorescence fluctuation spectroscopy in reduced detection volumes. Current Pharmaceutical Biotechnology, vol. 7, pp. 51-66, 2006.

[75] Madge, D. Chemical kinetics and fluorescence correlation spectroscopy. Q Rev Biophys, vol. 9 (1), pp. 35-47, 1976.

[76] Schwille, P., Bieschke, J., and Oehlenschlager, F. Kinetic investigations by fluorescence correlation spectroscopy: The analytical and diagnostic potential of diffusion studies. Biophysical Chemistry, vol. 66 (2-3), pp. 211-228, 1997.

[77] Haustein, E. and Schwille, P. Fluorescence correlation spectroscopy: Novel variations of an established technique. Annual Review of Biophysics and Biomolecular Structure, vol. 36, pp. 151-169, 2007. 
[78] Schwille, P., Korlach, J., and Webb, W. W. Fluorescence correlation spectroscopy with single-molecule sensitivity on cell and model membranes. Cytometry, vol. 36 (3), pp. 176-182, 1999.

[79] Saxton, M. J. Anomalous Diffusion Due to Obstacles - a Monte-Carlo Study. Biophysical Journal, vol. 66 (2), pp. 394-401, 1994.

[80] Wachsmuth, M., Waldeck, W., and Langowski, J. Anomalous diffusion of fluorescent probes inside living cell nuclei investigated by spatially-resolved fluorescence correlation spectroscopy. Journal of Molecular Biology, vol. 298 (4), pp. 677-689, 2000.

[81] Eggeling, C., Ringemann, C., Medda, R., Schwarzmann, G., Sandhoff, K., Polyakova, S., Belov, V. N., Hein, B., von Middendorff, C., Schonle, A., and Hell, S. W. Direct observation of the nanoscale dynamics of membrane lipids in a living cell. Nature, vol. 457 (7233), pp. 1159-U121, 2009.

[82] Kinoshita, T., Fujita, M., and Maeda, Y. Biosynthesis, remodelling and functions of mammalian GPI-anchored proteins: Recent progress. Journal of Biochemistry, vol. 144 (3), pp. 287-294, 2008.

[83] Simons, K. and Ikonen, E. Functional rafts in cell membranes. Nature, vol. 387 (6633), pp. 569-572, 1997.

[84] Jacobson, K., Mouritsen, O. G., and Anderson, R. G. W. Lipid rafts: at a crossroad between cell biology and physics. Nature Cell Biology, vol. 9 (1), pp. 7-14, 2007.

[85] Munro, S. Lipid rafts: Elusive or illusive? Cell, vol. 115 (4), pp. 377-388, 2003.

[86] Shaw, A. S. Lipid rafts: now you see them, now you don't. Nature Immunology, vol. 7 (11), pp. 1139-1142, 2006.

[87] Lenne, P. F., Wawrezinieck, L., Conchonaud, F., Wurtz, O., Boned, A., Guo, X. J., Rigneault, H., He, H. T., and Marguet, D. Dynamic molecular confinement in the plasma membrane by microdomains and the cytoskeleton meshwork. Embo Journal, vol. 25 (14), pp. 3245-3256, 2006.

[88] Schwille, P., MeyerAlmes, F. J., and Rigler, R. Dual-color fluorescence crosscorrelation spectroscopy for multicomponent diffusional analysis in solution. Biophysical Journal, vol. 72 (4), pp. 1878-1886, 1997.

[89] Szent-Gyorgyi, C., Schmidt, B. A., Creeger, Y., Fisher, G. W., Zakel, K. L., Adler, S., Fitzpatrick, J. A. J., Woolford, C. A., Yan, Q., Vasilev, K. V., Berget, P. B., Bruchez, M. P., Jarvik, J. W., and Waggoner, A. Fluorogen-activating single-chain antibodies for imaging cell surface proteins. Nature Biotechnology, vol. 26 (2), pp. 235-240, 2008. 


\section{List of publications}

Parts of this thesis have been published as follows:

- Hein, B., Willig, K. I. and Hell, S. W., Stimulated emission depletion (STED) nanoscopy of a fluorescent protein-labeled organelle inside a living cell, Proceedings of the National Academy of Sciences of the United States of America, vol. 105, 38, pp 1427114276, 2008.

- Eggeling, C., Ringemann, C., Medda, R., Schwarzmann, G., Sandhoff, K., Polyakova, S., Belov, V. N., Hein, B.; von Middendorff, C., Schonle, A. and Hell, S. W., Direct observation of the nanoscale dynamics of membrane lipids in a living cell, Nature, vol. 457, 7233, pp 1159-U121, 2009.

- Naegerl, V., Willig, K., Hein, B., Hell, S. and Bonhoeffer, T., Live-cell imaging of dendritic spines by STED microscopy, Proceedings of the National Academy of Sciences of the United States of America, vol. 105, 48, pp 18982-18987, 2008.

- Fölling, J., Bossi, M., Bock, H., Medda, R., Wurm, C. A., Hein, B., Jakobs, S., Eggeling, C. and Hell, S. W., Fluorescence nanoscopy by ground-state depletion and single-molecule return, Nature Methods, vol. 5, 11, pp 943-945, 2008.

- Willig, K. I., Kellner, R. R., Medda, R., Hein, B., Jakobs, S. and Hell, S. W., Nanoscale resolution in GFP-based microscopy, Nature Methods, vol. 3, 9, pp 721-723, 2006.

- Boyarskiy, V. P., Belov, V. N., Medda, R., Hein, B., Bossi, M. and Hell, S. W., Photostable, amino reactive and water-soluble fluorescent labels based on sulfonated rhodamine with a rigidized xanthene fragment, Chemistry-a European Journal, vol. 14, 6, pp 1784-1792, 2008.

- Hein, B. Willig, I. Wurm, C., Jakobs, S., Hell, S., Usage of a genetically encoded tag binding organic dyes broadens the application range of live-cell STED microscopy, in preparation. 


\section{Acknowledgement}

I want to use the opportunity to thank all those people who have contributed to the success of this work:

- Prof. Stefan Hell for giving the opportunity to work on this project, his encouragement and input, and for providing an outstanding lab environment

- Prof. Jürgen Troe for his interest in this work and his willingness to be the main referee

- Katrin Willig for teaching me everything there is to know about STED

- Christian Eggeling for suggestions, help, and support in the FCS measurements

- Sylvia Löbermann for showing me everything a hobby-biologist needs to know

- Rebecca Medda for the ongoing introduction into cell culture

- Tanja Gilat for thousands of beautiful cells

- Christian Wurm for manymany plasmids, yeast and from time to time the ride uphill

- Jaydev Jethwa for help with lasers, heating, cooling and the smaller and larger technical problems

- Vladimir Belov and his team for chemical advice and synthesis

- Benjamin Harke for the Atto647N-measurements

- Gael Moneron, Volker Westphal, Andreas Schönle and Jan Keller for help with the setup, the software, and the analysis

- Jochen Sieber for the Venus-labeled samples

- Christian Ringemann for help with the FCS measurements

- Stefan Jakobs and all biologists for biological advise

- Dominik Wildanger for sharing his $\mathrm{LT}_{\mathrm{E} X} \mathrm{X}$ expertise

- Katrin Willig, Christian Eggeling, Claas v. Middendorf, Benjamin Harke, and Chaitanya Ullal for proofreading parts of the manuscript 
- Sarah Aschemann and Joesephine Stadler for making the impossible possible

- Marco Roose for his patience with me and my computer

- The mechanical and optical workshop for many (same-day) home-built parts

- All the people who made the time here with sports, music, food, and discussions very enjoyable and unforgettable

- My family, especially Niko and my parents, for their patience and support 


\section{Lebenslauf}

Persönliche Daten

$\begin{array}{ll}\text { Name } & \text { Hein, Birka } \\ \text { Geburtsdatum } & 13.02 .1980 \\ \text { Geburtsort } & \text { Celle }\end{array}$

Schulausbildung

$\begin{array}{ll}1986-1990 & \text { Grundschule Mellendorf } \\ 1990-1992 & \text { Orientierungsstufe Mellendorf } \\ 1992-1999 & \text { Gymnasium Mellendorf }\end{array}$

Studium

1999 - $2001 \quad$ Grundstudium Chemie, Universität Bielefeld

Grundstudium Biochemie, Universität Bielefeld

2002 - $2003 \quad$ Ohio State Biochemistry Program, Ohio State University

$2001-2005 \quad$ Hauptstudium Chemie, Universität Bielefeld

Seit $2005 \quad$ Promotion am Max-Planck-Institut für biophysikalische

Chemie, Abteilung NanoBiophotonik

Göttingen, Mai 2009

Birka Hein 